\title{
Estimated Use of Water in the United States in 2010
}

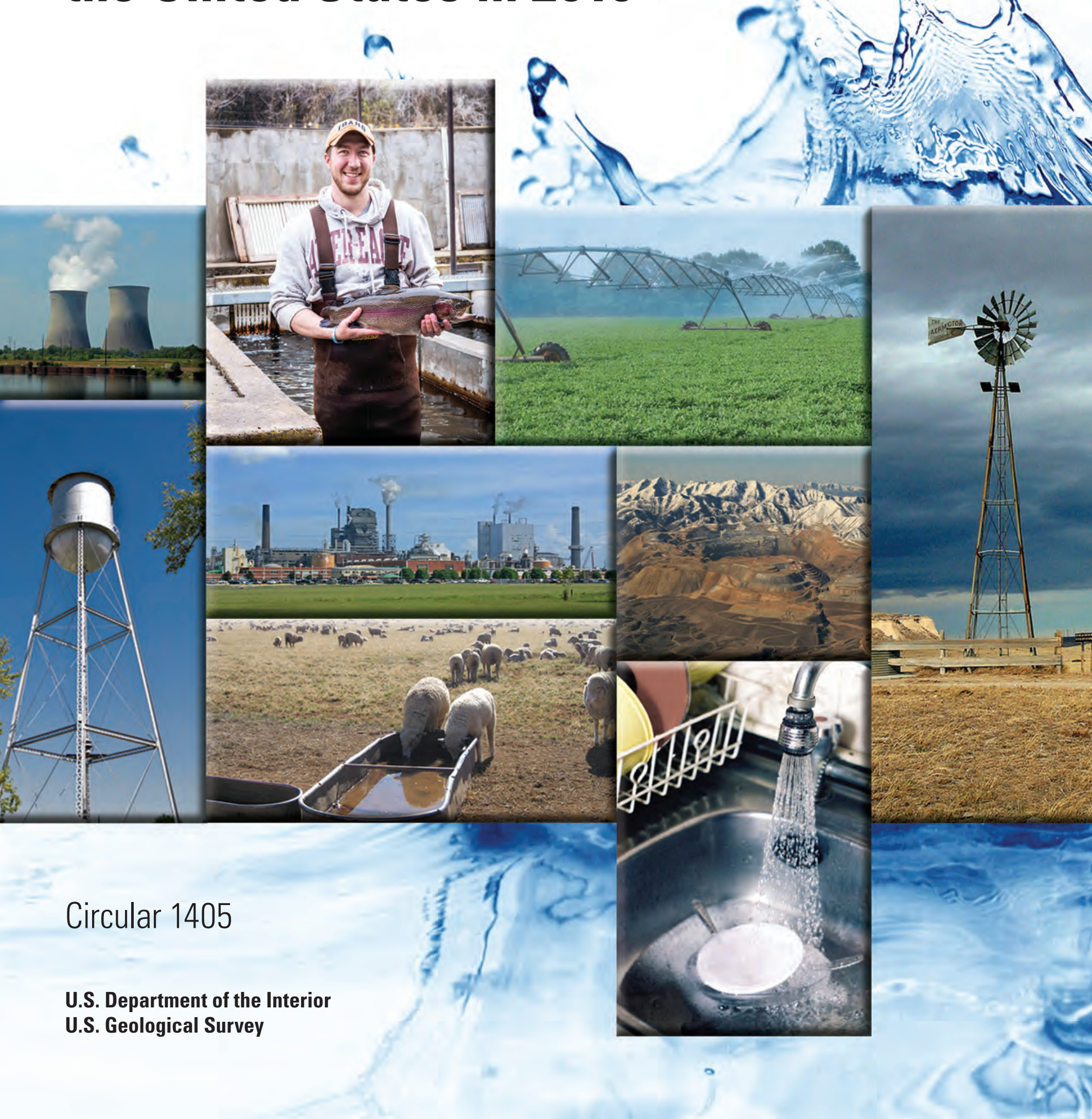




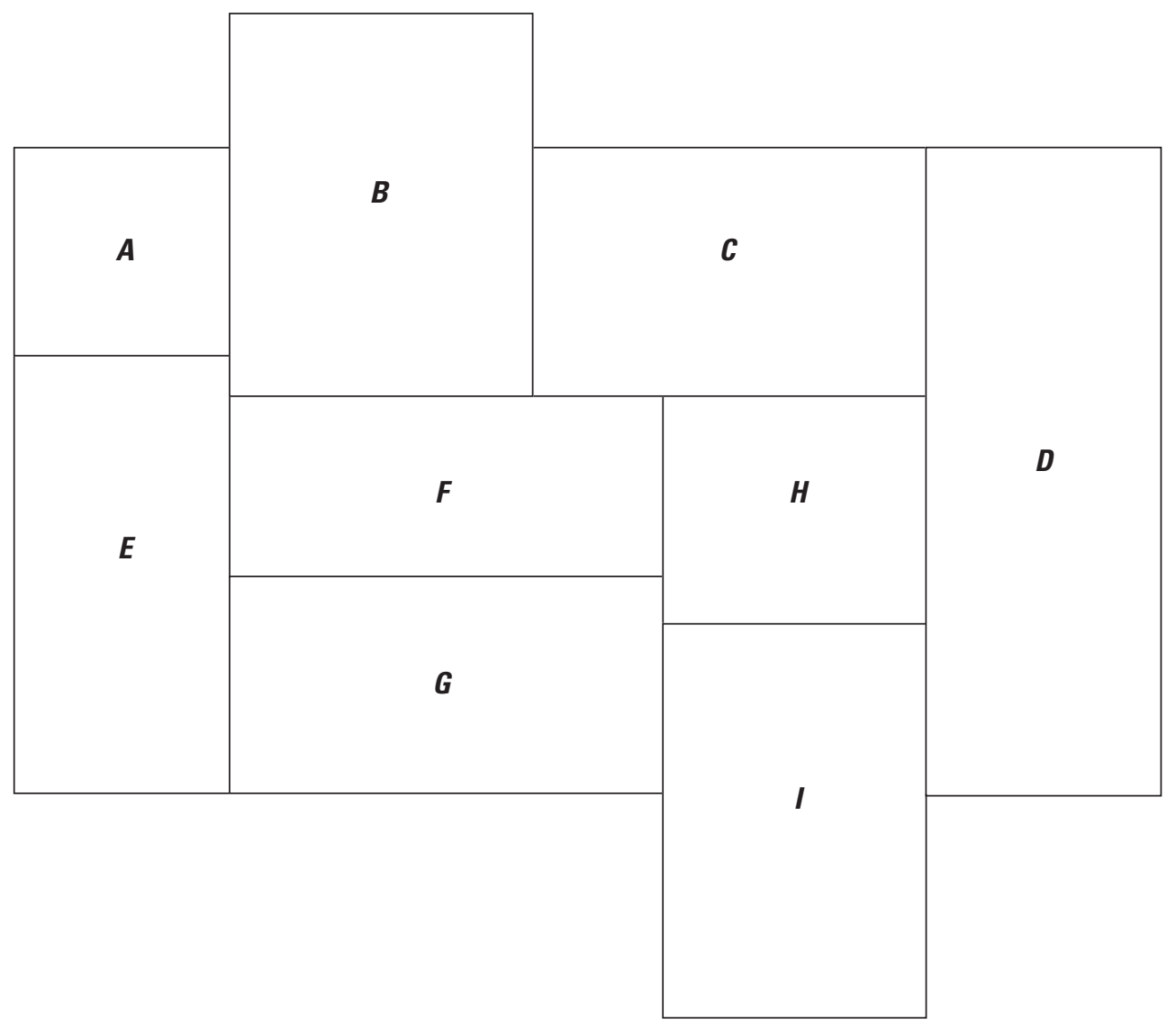

Front cover. Photo collage of water use and supply. $\boldsymbol{A}$, Watts Bar Nuclear Powerplant, Rhea County, Tennessee (photo by Alan Cressler, USGS). $\boldsymbol{B}$, Student with trout at the Aquaculture Research Institute, Hagerman, Idaho (photo from University of Idaho, used with permission). C, Center-pivot irrigation, Mitchell County, Georgia (photo by Alan Cressler, USGS). D, Windmill on Pawnee Butte Grasslands (photo by Ray Klocek, used with permission). $\boldsymbol{E}$, Water tower in Council, Idaho, Adams County (photo by Justin Woody, used with permission). $\boldsymbol{F}$, Pulp mill, Wayne County, Georiga (photo by Alan Cressler, USGS). G, Sheep at water trough on the open range (photo by Saeid Tadayon, USGS). H, Bingham Canyon Mine, Salt Lake County, Utah (photo by Alan Cressler, USGS). I, Domestic water use in the kitchen (photo from Wikimedia Commons). 


\section{Estimated Use of Water in the United States in 2010}

By Molly A. Maupin, Joan F. Kenny, Susan S. Hutson, John K. Lovelace, Nancy L. Barber, and Kristin S. Linsey

Circular 1405 


\title{
U.S. Department of the Interior SALLY JEWELL, Secretary
}

\section{U.S. Geological Survey \\ Suzette M. Kimball, Acting Director}

\author{
U.S. Geological Survey, Reston, Virginia: 2014
}

For more information on the USGS - the Federal source for science about the Earth, its natural and living resources, natural hazards, and the environment—visit http://WwW.usgs.gov or call 1-888-ASK-USGS

For an overview of USGS information products, including maps, imagery, and publications, visit $h t t p: / / w w w . u s g s . g o v / p u b p r o d$

To order this and other USGS information products, visit http://store.usgs.gov

Any use of trade, product, or firm names is for descriptive purposes only and does not imply endorsement by the U.S. Government.

Although this information product, for the most part, is in the public domain, it also may contain copyrighted materials as noted in the text. Permission to reproduce copyrighted items must be secured from the copyright owner.

Suggested citation:

Maupin, M.A., Kenny, J.F., Hutson, S.S., Lovelace, J.K., Barber, N.L., and Linsey, K.S., 2014, Estimated use of water in the United States in 2010: U.S. Geological Survey Circular 1405, 56 p., http://dx.doi.org/10.3133/cir1405.

\section{Library of Congress Cataloging-in-Publication Data}

Maupin, Molly A., author.

Estimated use of water in the United States in 2010 / by Molly A. Maupin, Joan F. Kenny, Susan S. Hutson, John K. Lovelace, Nancy L. Barber, and Kristin S. Linsey.

pages cm. -- (Circular ; 1405)

Includes bibliographical references.

ISBN 978-1-4113-3862-3 (pbk. : alk. paper)

1. Water consumption--United States. I. Kenny, J. F., author. II. Hutson, Susan S., author. III. Lovelace, John K., author. IV. Barber, Nancy L., author. V. Linsey, Kristin S., author. VI. Geological Survey (U.S.), issuing body. VII. Title. VIII. Series: U.S. Geological Survey circular ; 1405.

TD223.M365 2015

333.91'130973--dc23

2014041921

ISSN 1067-084X (Print)

ISSN 2330-5703 (Online) 


\section{Contents}

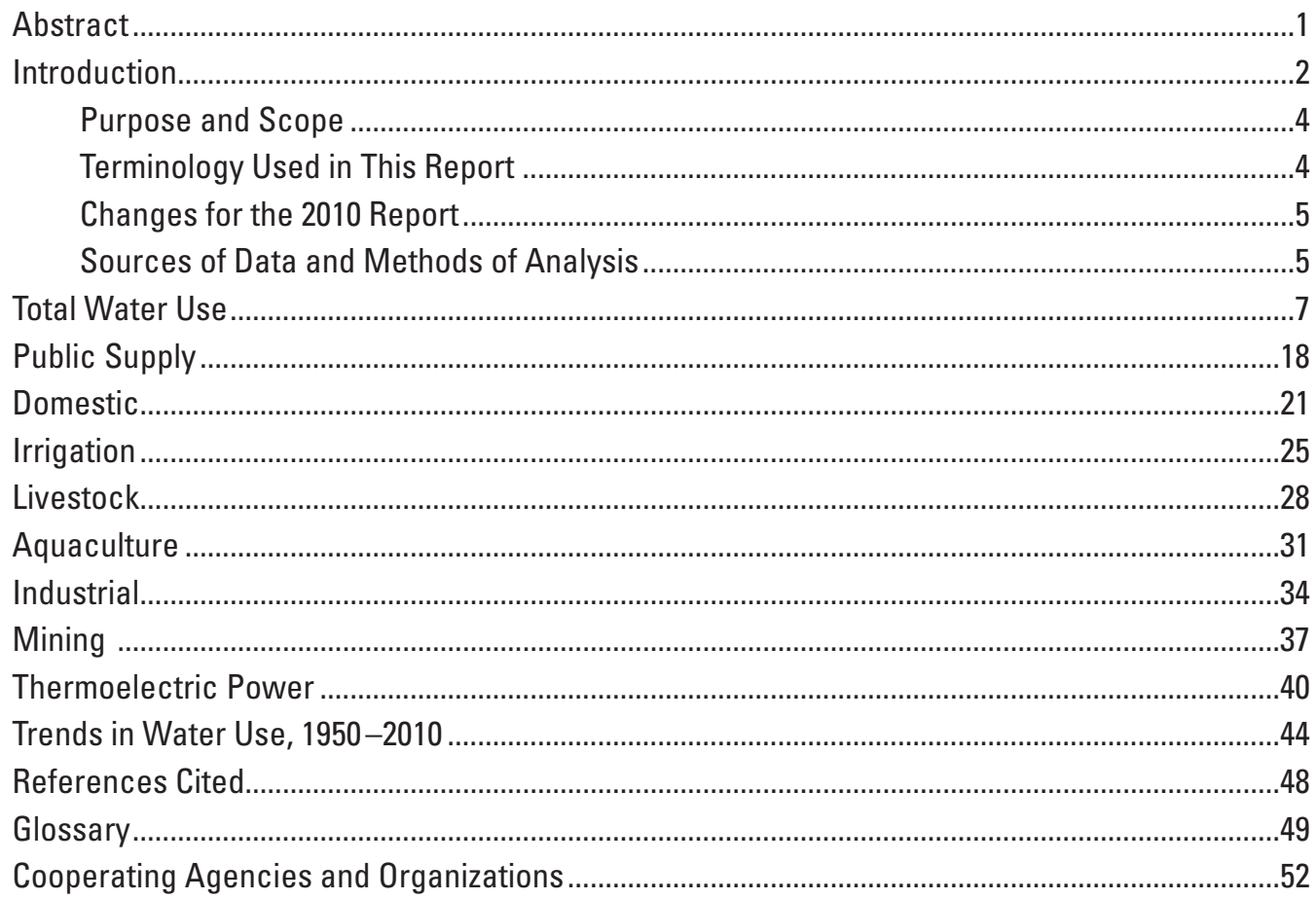

\section{Acknowledgments}

This national compilation of water use would not be possible without the assistance and data provided by the many State and local agencies that manage water resources, operate datacollection programs, and administer regulations for use of water and other natural resources. The agencies and other organizations that provided assistance are listed for each State at the end of this report. The authors also gratefully acknowledge the USGS personnel in each State who compiled the data for this report. 
Conversion Factors

\begin{tabular}{|c|c|c|}
\hline Multiply & By & To obtain \\
\hline \multicolumn{3}{|c|}{ Area } \\
\hline acre & 4,047 & square meter $\left(\mathrm{m}^{2}\right)$ \\
\hline acre & 0.4047 & hectare (ha) \\
\hline acre & 0.001562 & square mile $\left(\mathrm{mi}^{2}\right)$ \\
\hline \multicolumn{3}{|c|}{ Volume } \\
\hline acre-foot (acre-ft) & 1,233 & cubic meter $\left(\mathrm{m}^{3}\right)$ \\
\hline acre-foot (acre-ft) & 325,851 & gallon (gal) \\
\hline acre-foot (acre-ft) & 43,560 & cubic foot $\left(\mathrm{ft}^{3}\right)$ \\
\hline cubic foot $\left(\mathrm{ft}^{3}\right)$ & 7.48 & gallon (gal) \\
\hline gallon (gal) & 3.785 & liter $(\mathrm{L})$ \\
\hline gallon (gal) & 3.785 & cubic decimeter $\left(\mathrm{dm}^{3}\right)$ \\
\hline million gallons (Mgal) & 3,785 & cubic meter $\left(\mathrm{m}^{3}\right)$ \\
\hline million gallons (Mgal) & 3.07 & acre-foot (acre-ft) \\
\hline \multicolumn{3}{|c|}{ Flow rate } \\
\hline acre-foot per year (acre-ft/yr) & 1,233 & cubic meter per year $\left(\mathrm{m}^{3} / \mathrm{yr}\right)$ \\
\hline billion gallons per day (Bgal/d) & 1.3815 & billion cubic meters per year \\
\hline gallon per day (gal/d) & 3.785 & liter per day (L/d) \\
\hline million gallons per day (Mgal/d) & 0.04381 & cubic meter per second $\left(\mathrm{m}^{3} / \mathrm{s}\right)$ \\
\hline million gallons per day (Mgal/d) & 1.547 & cubic foot per second $\left(\mathrm{ft}^{3} / \mathrm{sec}\right)$ \\
\hline million gallons per day (Mgal/d) & 1.121 & $\begin{array}{l}\text { thousand acre-feet per year } \\
\quad(\text { acre-ft/yr) }\end{array}$ \\
\hline million gallons per day (Mgal/d) & 1.3815 & million cubic meters per year \\
\hline thousand acre-feet per year (acre-ft/yr) & 0.8921 & million gallons per day (Mgal/d) \\
\hline \multicolumn{3}{|c|}{ Energy } \\
\hline gigawatt-hour (gWh) & $3,600,000$ & Megajoule (MJ) \\
\hline kilowatt-hour (kWh) & $3,600,000$ & joule (J) \\
\hline
\end{tabular}

\section{Abbreviations}

$\begin{array}{ll}\text { EPA } & \text { U.S. Environmental Protection Agency } \\ \text { NWC } & \text { National Water Census } \\ \text { NWUIP } & \text { National Water Use Information Program } \\ \text { SDWIS } & \text { Safe Drinking Water Information System } \\ \text { SECURE } & \text { Science and Engineering to Comprehensively Understand and Responsibly Enhance } \\ \text { USDA ARS } & \text { U.S. Department of Agriculture, Agricultural Research Service } \\ \text { USDA NASS } & \text { U.S. Department of Agriculture, National Agricultural Statistics Service } \\ \text { USDA NRCS } & \text { U.S. Department of Agriculture, Natural Resources Conservation Service } \\ \text { USDOE EIA } & \text { U.S. Department of Energy, Energy Information Administration } \\ \text { USGS } & \text { U.S. Geological Survey }\end{array}$




\title{
Estimated Use of Water in the United States in 2010
}

\author{
By Molly A. Maupin, Joan F. Kenny, Susan S. Hutson, John K. Lovelace, \\ Nancy L. Barber, and Kristin S. Linsey
}

\section{Abstract}

Water use in the United States in 2010 was estimated to be about 355 billion gallons per day (Bgal/d), which was 13 percent less than in 2005 . The 2010 estimates put total withdrawals at the lowest level since before 1970. Freshwater withdrawals were $306 \mathrm{Bgal} / \mathrm{d}$, or 86 percent of total withdrawals, and saline-water withdrawals were $48.3 \mathrm{Bgal} / \mathrm{d}$, or 14 percent of total withdrawals. Fresh surface-water withdrawals $(230 \mathrm{Bgal} / \mathrm{d})$ were almost 15 percent less than in 2005 , and fresh groundwater withdrawals (76.0 Bgal/d) were about 4 percent less than in 2005. Saline surface-water withdrawals were $45.0 \mathrm{Bgal} / \mathrm{d}$, or 24 percent less than in 2005 . Updates to the 2005 saline groundwater withdrawals, mostly for thermoelectric power, reduced total saline groundwater withdrawals to $1.51 \mathrm{Bgal} / \mathrm{d}$, down from the originally reported $3.02 \mathrm{Bgal} / \mathrm{d}$. Total saline groundwater withdrawals in 2010 were $3.29 \mathrm{Bgal} / \mathrm{d}$, mostly for mining use.

Thermoelectric power and irrigation remained the two largest uses of water in 2010, and total withdrawals for both were notably less than in 2005. Withdrawals in 2010 for thermoelectric power were 20 percent less and withdrawals for irrigation were 9 percent less than in 2005. Similarly, other uses showed reductions compared to 2005 , specifically public supply ( -5 percent), self-supplied domestic ( -3 percent), selfsupplied industrial ( -12 percent), and livestock ( -7 percent). Only mining (39 percent) and aquaculture ( 7 percent) reported larger withdrawals in 2010 compared to 2005. Thermoelectric power, irrigation, and public-supply withdrawals accounted for 90 percent of total withdrawals in 2010 .

Withdrawals for thermoelectric power were $161 \mathrm{Bgal} / \mathrm{d}$ in 2010 and represented the lowest levels since before 1970 . Surface-water withdrawals accounted for more than 99 percent of total thermoelectric-power withdrawals, and 73 percent of those surface-water withdrawals were from freshwater sources. Saline surface-water withdrawals for thermoelectric power accounted for 97 percent of total saline surface-water withdrawals for all uses. Thermoelectric-power withdrawals accounted for 45 percent of total withdrawals for all uses, and freshwater withdrawals for thermoelectric power accounted for 38 percent of the total freshwater withdrawals for all uses.

Irrigation withdrawals were $115 \mathrm{Bgal} / \mathrm{d}$ in 2010 and represented the lowest levels since before 1965. Irrigation withdrawals, all freshwater, accounted for 38 percent of total freshwater withdrawals for all uses, or 61 percent of total freshwater withdrawals for all uses excluding thermoelectric power. Surface-water withdrawals (65.9 Bgal/d) accounted for 57 percent of the total irrigation withdrawals, or about 12 percent less than in 2005. Groundwater withdrawals were $49.5 \mathrm{Bgal} / \mathrm{d}$ in 2010, about 6 percent less than in 2005. About 62,400 thousand acres were irrigated in 2010, an increase from 2005 of about 950 thousand acres ( 1.5 percent). The number of acres irrigated using sprinkler and microirrigation systems continued to increase and accounted for 58 percent of the total irrigated lands in 2010.

Public-supply withdrawals in 2010 were $42.0 \mathrm{Bgal} / \mathrm{d}$, or 5 percent less than in 2005, and represented the first declines in public-supply withdrawals since the 5-year reporting began in 1950. Total population in the United States increased from 300.7 million people in 2005 to 313.0 million people in 2010, an increase of 4 percent. Public-supply withdrawals accounted for 14 percent of the total freshwater withdrawals for all uses and 22 percent of freshwater withdrawals for all uses excluding thermoelectric power. The number of people that received potable water from public-supply facilities in 2010 was 268 million, or about 86 percent of the total U.S. population. This percentage was unchanged from 2005. Selfsupplied domestic withdrawals were $3.60 \mathrm{Bgal} / \mathrm{d}$, or 3 percent less than in 2005. More than 98 percent of the self-supplied domestic withdrawals were from groundwater sources.

Self-supplied industrial withdrawals were $15.9 \mathrm{Bgal} / \mathrm{d}$ in 2010, a 12 percent decline from 2005, and continued the downward trend since the peak of $47 \mathrm{Bgal} / \mathrm{d}$ in 1970 . Total self-supplied industrial withdrawals were 4 percent of total withdrawals for all uses and 8 percent of total withdrawals for all uses excluding thermoelectric power. Most of the total 
self-supplied industrial withdrawals were from surface-water sources ( 82 percent), and nearly all (93 percent) of those surface-water withdrawals were from freshwater sources. Nearly all of the groundwater withdrawals for self-supplied industrial use (98 percent) were from freshwater sources.

Total aquaculture withdrawals were $9.42 \mathrm{Bgal} / \mathrm{d}$ in 2010, or 7 percent more than in 2005, and surface water was the primary source ( 81 percent). Most of the surface-water withdrawals occurred at facilities that operated flowthrough raceways, which returned the water to the source directly after use. Aquaculture withdrawals accounted for 3 percent of the total withdrawals for all uses and 5 percent of the total withdrawals for all uses excluding thermoelectric.

Total mining withdrawals in 2010 were $5.32 \mathrm{Bgal} / \mathrm{d}$, or about 1 percent of total withdrawals from all uses and 3 percent of total withdrawals from all uses excluding thermoelectric. Mining withdrawals accounted for the largest percentage increase (39 percent) in water use between 2005 and 2010 among all the categories. Groundwater withdrawals accounted for 73 percent of the total mining withdrawals, and the majority of the groundwater was saline (71 percent). The majority ( 80 percent) of surface-water withdrawals for mining was freshwater.

Livestock withdrawals in 2010 were $2.00 \mathrm{Bgal} / \mathrm{d}$, or 7 percent less than in 2005. All livestock withdrawals were from freshwater sources, mostly from groundwater (60 percent). Livestock withdrawals accounted for about 1 percent of total freshwater withdrawals for all uses excluding thermoelectric power.

In 2010, more than 50 percent of the total withdrawals in the United States were accounted for by 12 States. California accounted for about 11 percent of the total withdrawals and 10 percent of freshwater withdrawals in the United States, predominantly for irrigation. Texas accounted for about 7 percent of total withdrawals, predominantly for thermoelectric power, irrigation, and public supply. Florida accounted for 18 percent of the total saline-water withdrawals in the United States, mostly from surface-water sources for thermoelectric power. Oklahoma and Texas accounted for about 70 percent of the total saline groundwater withdrawals in the United States, mostly for mining.

\section{Introduction}

This report, "Estimated use of water in the United States in 2010," is the 13th in a series of U.S. Geological Survey (USGS) Circular reports that have been published every 5 years since 1950 . The 60 -year span of national reports represents the longest compilation record of water-use data by a Federal agency in the United States. Estimates of withdrawals enable the depiction of trends in total water use for the Nation among different geographic areas, categories of use, and sources over time. The USGS is dedicated to providing reliable scientific information that accurately describes current and historic conditions and enables a better understanding of the Earth's precious water resources. Water-use information complements and supports surfacewater and groundwater availability studies and water budgets that are critical to these studies. This information is also essential to accurately understand how future water demands will be met while maintaining adequate water quality and quantities for human and ecosystem needs.

The National Water Use Information Program (NWUIP) is the USGS program (http://water.usgs.gov/watuse/) that facilitates the 5-year compilation of water use and over time has met various challenges in estimating water use in the United States. The program, however, has reduced some data collections over time to address limitations of available resources for analysis and limitations of capabilities for accurate interpolations. The National Water Census (NWC) is a recent USGS program, implemented as part of the SECURE (Science and Engineering to Comprehensively Understand and Responsibly Enhance) Water Act (Subtitle F of Public Law 111-11, the Omnibus Public Land Management Act) to study national water availability and use by integrating diverse research and building new water accounting tools, such as decision support capacity. These tools and research are designed to enable water managers to accurately assess water availability at regional and national scales (http://water.usgs.gov/watercensus/). To meet NWC goals of building water budget assessments at regional and national scales, accurate and complete water-use estimates are necessary. The NWUIP is working closely with the NWC to provide water-use data for accurate water budget assessments in the NWC study areas. To meet these goals, several water-use specific research studies supported by the NWC were begun, some are completed, and some are ongoing. Each study specifically addresses a water-use data collection challenge, such as improvement in the dissemination of information on data inventories, collection of more accurate information, use of better methods for analysis, and upgrade of data dissemination tools.

NWC-supported projects with direct relevance to water use were conducted concurrently with the NWUIP 2010 compilation efforts and focused on the three largest categories of water use, irrigation, thermoelectric power, and public supply. For irrigation water use, methods and documentation were synthesized into a national report using the 2000 and 2005 compilation data and suggested improved estimation methods (Dickens and others, 2011). Additionally, methods were developed to assist in estimating irrigation water use in humid Eastern States, using two predictive models that use climate, soils, and crop data to explain the potential for irrigation (Levin and Zarriello, 2013). For thermoelectric power, linked heat and water budget models were developed for 1,290 thermoelectric powerplants in the United States (Diehl and others, 2013). This project entailed a indepth inventory of powerplants and associated information. Data from this project considerably improved 


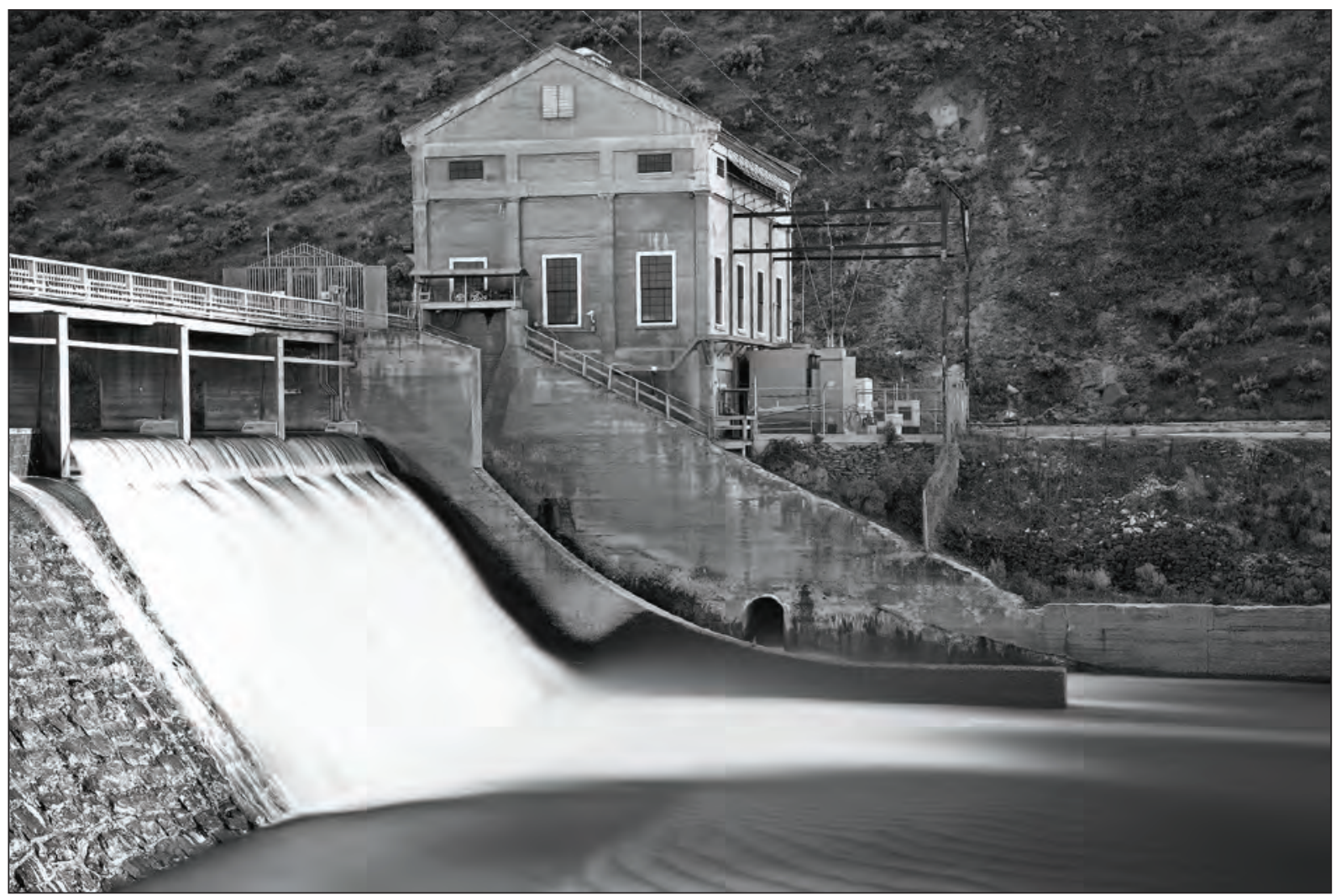

The Boise River Diversion Dam in Ada County, Idaho, was completed in 1909 and diverts water into the New York Canal, the primary irrigation canal for Ada and Canyon Counties. Photo by Jeff Woody, used with permission.

the NWUIP understanding of the cooling systems used at individual powerplants as well as provided a more complete inventory of powerplant locations and net power generation. On the basis of the water budget models, Diehl and Harris (2014) reported powerplant-specific estimates of withdrawals and consumptive use. For public supply, the U.S. Environmental Protection Agency (EPA) provided a publicsupply dataset from the Safe Drinking Water Information System (SDWIS). These data included site-specific well, surface-water intake, and distribution-system information, which was filtered through a USGS database (Price and Maupin, 2014) and enhanced for quality control using associated USGS data. These data were disseminated as State datasets to each USGS Water Science Center to help construct a site-specific database capable of storing public-supply withdrawal, distribution, use, and return data for each State.

Data dissemination capabilities and data-collection efforts have improved over the course of each 5-year compilation. The online resource, "USGS Water Use Data for the Nation" (http://waterdata.usgs.gov/nwis/wu), provides the best available county water-use data (1985-2010). These county-level estimates are the foundation for the statewide totals presented in each 5-year compilation report and are stored, updated, and disseminated using the USGS National Water Information System (NWIS) database. Data are retrievable as county, State, and national totals for each category of use as reported in the
5 -year compilation reports. Because data are updated periodically and revised during interim years, the Web site will enable quick and easy access to the most current water-use data.

Factors such as demographics, new manufacturing and cooling-system technologies, economic trends, legal decisions, and climatic fluctuations have varying effects on water use. Between 2000 and 2010, population growth in the U.S. was 9.7 percent, lower than the 13.2 percent growth for the 1990-2000 period (U.S. Census Bureau, 2011). More population growth was recorded in Southern and Western States (14.3 and 13.8 percent, respectively) between 2000 and 2010 compared to Midwestern States (3.9 percent) and Northeastern States (3.2 percent). Southern and Western States accounted for more than 84 percent of the total U.S. population growth from 2000 to 2010. Population growth puts additional pressure on existing public utilities and increases demand on sometimes already limited water supplies. In parts of the United States, communities have sought additional water sources or instituted water-conservation measures to meet increasing demands. New cooling-system technologies and wastewater management practices at thermoelectric powerplants and industrial facilities are examples of water-saving practices that are being implemented. Powerplants have reduced the demand for cooling water by implementing more efficient cooling systems, such as changing to recirculating systems or building new plants with dry-cooling systems. Industrial facilities are 
using more efficient water-conserving manufacturing technologies, driven by higher costs for water and energy. Industrial manufacturing has declined with more goods being produced outside of the United States. Increases in industrial reuse and recycling of wastewater help to reduce withdrawals from the available resources and treated discharges to surface waters over time.

Climate fluctuations affect water use, particularly for irrigation, power generation, and public supply. In 2010, the contiguous United States (CONUS) experienced average annual air temperatures slightly above normal and precipitation above the long-term average. An abnormally cold winter with abundant moisture resulted in record-breaking precipitation in the East and Northeast for December-February. While the East enjoyed an abnormally warm spring, the Western United States experienced below normal temperatures. The summer of 2010 was the fourth warmest on record for the CONUS, but was the ninth wettest in 116 years in the Upper Midwest and Great Lakes. The West and Southeast had below-normal precipitation during the summer. The fall of 2010 was warmer than normal, but the Upper Midwest and Northeast continued to receive above-average precipitation, while Florida suffered through the second driest September-November period on record (National Oceanic and Atmospheric Administration National Climatic Data Center, 2010).

Cooling-system technology in thermoelectric powerplants has dramatically improved in recent years, causing large changes in withdrawals between 2005 and 2010. Improvements driven by the Clean Water Act and other economic factors have changed the way industrial facilities use, reuse, and recycle water, resulting in reduced discharges to wastewater-treatment plants or surface-water bodies. Cooling water is essential for producing most of the thermoelectric power in the United States, and an increase in electric energy use has resulted in additional demands for water. Limitations on water supplies have led to the use of less water-intensive cooling technologies for producing thermoelectric power in newer powerplants.

\section{Purpose and Scope}

This report presents average daily withdrawals (in millions of gallons per day) for calendar year 2010, by source (groundwater and surface water) and quality (fresh and saline) for the 50 States, the District of Columbia, Puerto Rico, and the U.S. Virgin Islands (hereafter referred to as "States" for brevity). Withdrawals are reported by category of use: public supply, domestic (including self-supplied domestic and deliveries from public supply), irrigation, livestock, aquaculture, self-supplied industrial (referred to as "industrial" for brevity), mining, and self-supplied thermoelectric power (referred to as "thermoelectric power" for brevity). Saline water is defined as water containing dissolved solids of
1,000 milligrams per liter or more. All withdrawals for the public supply, domestic, irrigation, and livestock categories are reported as totals, although in some areas water is treated to reduce salinity for these uses. Aquaculture totals include a small amount of saline surface-water withdrawals for two States. Both freshwater and saline-water withdrawals are reported for industrial, mining, and thermoelectricpower uses.

The series of 5-year national water-use estimates compiled by the USGS serves as one of the few sources of information about regional and national trends in water withdrawals. These historical reports (MacKichan, 1951, 1957; MacKichan and Kammerer, 1961; Murray, 1968; Murray and Reeves, 1972, 1977; Solley and others, 1983, 1988, 1993, 1998; Hutson and others, 2004, Kenny and others, 2009) are available online at http://water.usgs.gov/watuse/50years.html. Statewide data between 1950 and 2010 produced for the 5 -year national water-use estimates are available online at http://waterdata.usgs.gov/nwis/wu/. County-level data are available only for 1985-2010 from the same Web site

\section{Terminology Used in This Report}

A glossary of the terms and units used in this report is located at the end of the report and is available online at http://water.usgs.gov/watuse/wuglossary.html. Terms and units depicting withdrawals and ancillary data for the 5-year compilations have not changed since 2000 . Withdrawal for each category of use represents the total amount of water removed from the water source for a particular use, regardless of how much of that total is consumptively used or returned to the hydrologic system for future use. In most cases, some fraction of the total withdrawal will be returned to a water source after use and will be available for other subsequent uses. Consumptive use, however, precludes the subsequent withdrawal for another use, at least temporarily, because it represents that fraction of water that is removed from availability due to evaporation, transpiration, or incorporation into products or crop, or consumed by livestock or human. Estimates of return flows and consumptive use were discontinued after 1995, primarily because of resource and data constraints on the USGS National Water Use Information Program (NWUIP). Recent efforts by other programs in coordination with NWUIP have been implemented to reinstate the consumptive-use estimates for thermoelectric power and irrigation, but those data are not included in this report. Estimates of wastewater reuse were compiled by some States for the industrial, thermoelectric power, and irrigation categories, but these estimates were not included in the totals reported in tables in this report because of the small volumes of water compared to the totals and the incomplete reporting across the Nation.

Withdrawals are expressed in terms of millions of gallons per day and thousands of acre-feet per year. The term billions 
of gallons per day is used in the Abstract and Trends in Water Use sections of this report to more simply express large numbers for total uses. Units of millions or billions of gallons per day do not represent actual daily rates, but rather are used to express total amounts as an average daily rate over a single year. Water demands fluctuate seasonally and may be very different between hot summer months and cold winter months. Therefore, withdrawal estimates in this report represent the total annual withdrawals averaged over 365 days.

Withdrawals are rounded to three significant figures. All values are rounded independently, so the sums of individual rounded numbers may not equal the totals. The percentage of changes discussed in the text are calculated from the unrounded data and are expressed as integers. All population data are rounded to three significant figures. In discussions of States that compose the majority of withdrawals for a given category, the State names are listed in order of decreasing magnitude of withdrawals.

\section{Changes for the 2010 Report}

A matrix showing the different categories of use and how the terminology has changed over time is available online at http://water.usgs.gov/watuse/WU-Category-Changes.html. Links to definitions of water-use categories are included in the matrix. This report includes the same categories of use that were reported in 2005 , and every category of use includes data from every State. Some States may have compiled their estimates for livestock, aquaculture, or mining categories by using methods described by Lovelace (2009a, b). Similarly, some States may have compiled their estimates for thermoelectric power by using methods derived from Diehl and others (2013). Data from the NWC-supported thermoelectric-power study represent a substantial change in how data reported by Energy Information Administration (EIA) were used to estimate thermoelectric-power withdrawals. As in 2005, deliveries from public supply for domestic use were again compiled in 2010, but public-supply deliveries for commercial, industrial, and thermoelectric-power uses were not. Data were not compiled for hydrologic units (watersheds). Data were not compiled for commercial water use, hydroelectric-power generation, wastewater treatment (returns), consumptive use by category of use, and conveyance losses. Some of these additional data may have been collected by individual States but are not compiled as a national dataset or included in this report.

The Trends in Water Use section of this report includes national totals for withdrawals by category of use and source of water from 1950 to 2010 . Totals have changed for some categories and years because of revisions to individual State data during interim years. Because of these revisions, some of the percentage changes in this report will be slightly different from data published previously by Kenny and others (2009).

\section{Sources of Data and Methods of Analysis}

Data presented in this report were compiled from various sources, depending on the category of use and the information available for each State. USGS personnel in each State determined the best sources of information available, then compiled or estimated the data and prepared documentation of the sources and methods used to determine the water use totals. Data in this report may have been derived from reported, estimated, or calculated means using different sources and methods and, therefore, will have varying levels of accuracy. Because the largest users and the most prominent categories of use within each State have the greatest effect on the totals, obtaining reliable information for these large users and categories was the primary focus of the compilation effort. 
Sources of information used in the compilation include national datasets, State agency data, individual questionnaires, and local contacts. National datasets available to each State include the EPA SDWIS data (U.S. Environmental Protection Agency, 2014), U.S. Census Bureau population estimates (U.S. Census Bureau, 2011), U.S. Department of Agriculture (USDA) Farm and Ranch Irrigation Survey, USDA Census of Agriculture, USDA National Agricultural Statistics Service (NASS) crop and livestock estimates, including digital datasets derived from satellite imagery (Cropland Data Layer) for 2010, with associated confidence interval datasets, and U.S. Department of Energy (USDOE) EIA facility reports. Additional data for thermoelectric power, specifically locations and cooling-system classifications for powerplants, were provided from internal USGS sources (Diehl and others, 2013) using USDOE and project ancillary data. A list of industrial and commercial establishments was provided to USGS personnel from a commercial database for use in the industrial estimates. Datasets and sources of information used to produce the national estimates for the livestock, aquaculture, and mining categories include the USDA NASS, USDA county extension agents, USGS Minerals Information Team, USDOE EIA, and the U.S. Bureau of Mines. Sources of information are discussed in greater detail in the individual category sections of this report.

Many of these data, such as those from NASS and USDOE EIA, are collected annually. Other data are provided for years other than 2010, but were used to develop the 2010 estimates in some States because they were the most complete data available. For example, the USDA Census of Agriculture is produced in years ending in 2 and 7, and the USDA Farm and Ranch Irrigation Survey is produced in years ending in 3 and 8 . Correlation of water-use data in this report with specific climatic conditions for 2010 is not recommended because some data for years other than 2010 may have been used to develop some water-use estimates.

Guidelines for preparing the 2010 water-use estimates were distributed to USGS personnel in each Water Science Center through workshops, Web-based seminars, and written documents. The same guidelines used in 2005 were implemented in 2010 without change and are published as USGS Techniques and Methods Book 4, Chapter E1, "Guidelines for preparation of State water-use estimates for 2005" (Hutson, 2007). Reports published by individual USGS Water Science Centers as part of the NWUIP, as well as a list of contact personnel in each USGS Water Science Center, also are available online at $h$ ttp://water.usgs.gov/watuse/.

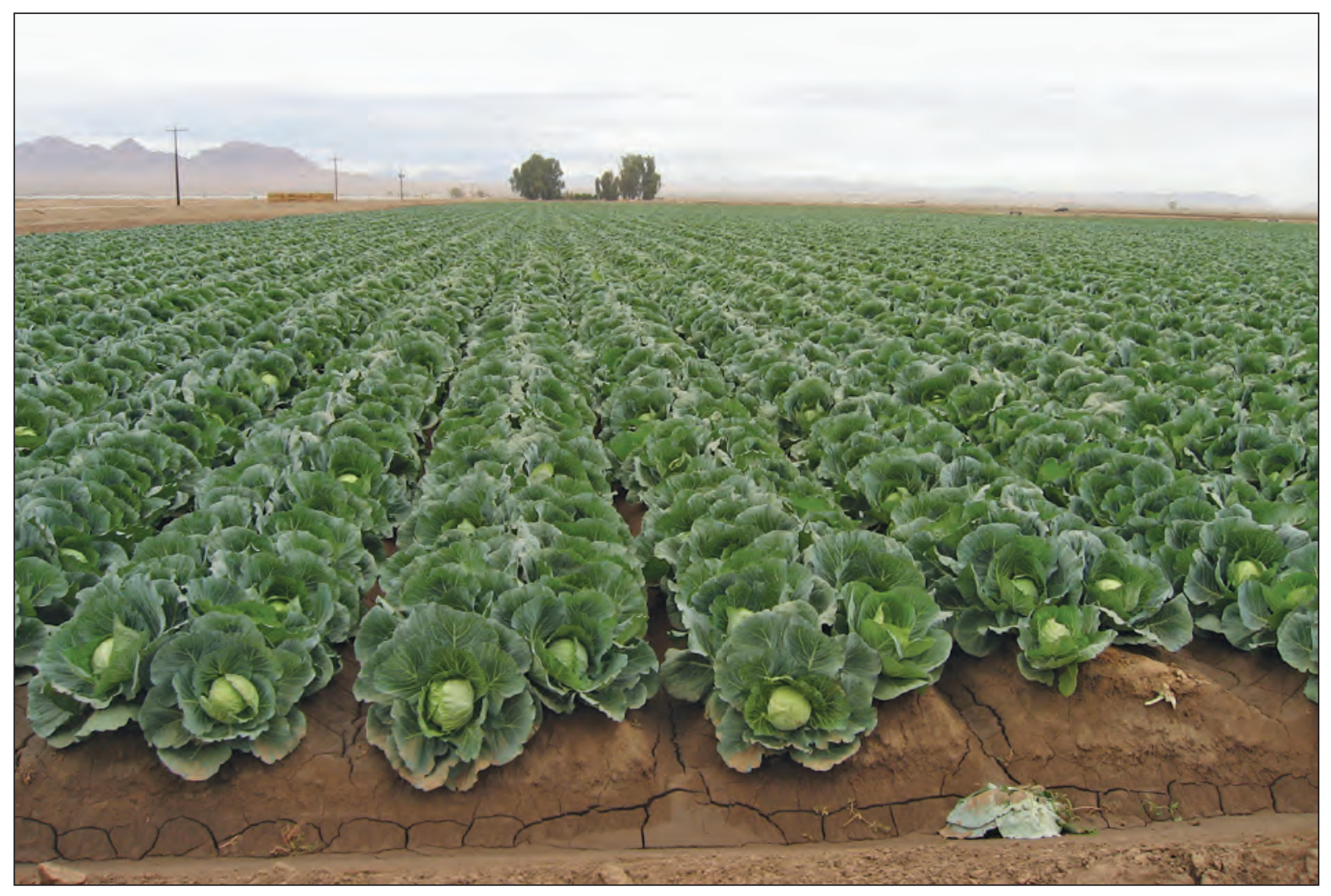

Cabbage field in Wellton Valley, Yuma County, Arizona. Photo by Saeid Tadayon, USGS. 
Total water withdrawals in the United States for 2010 were estimated for eight categories of use: public supply, domestic, irrigation, livestock, aquaculture, industrial, mining, and thermoelectric power (fig. 1). The three largest categories were thermoelectric power, irrigation, and public supply, cumulatively accounting for 90 percent of the national total. The remaining categories of industrial, aquaculture, mining, domestic, and livestock together were just about 10 percent of total water withdrawals estimated in this report.

Total State populations and withdrawals by source for 2010 are listed in table 1 . Total freshwater and saline-water withdrawals were estimated to be 355,000 million gallons per day $(\mathrm{Mgal} / \mathrm{d})$, or 397,000 thousand acre-feet per year (acre-ft/yr). Freshwater withdrawals of 306,000 Mgal/d made up 86 percent of the total, and saline-water withdrawals made up the remaining 48,300 Mgal/d (14 percent). Most salinewater withdrawals were seawater and brackish coastal water used for thermoelectric power. Total surface-water withdrawals were estimated to be $275,000 \mathrm{Mgal} / \mathrm{d}$, or 78 percent of the total. About 84 percent $(230,000 \mathrm{Mgal} / \mathrm{d})$ of total surface-water withdrawals were freshwater. Total groundwater withdrawals were $79,300 \mathrm{Mgal} / \mathrm{d}$, of which 96 percent (76,000 Mgal/d) was freshwater.

Total withdrawals by category and State are listed in table $2 A$, in million gallons per day, and in table $2 B$, in thousand acre-feet per year. Withdrawals for thermoelectric power $(161,000 \mathrm{Mgal} / \mathrm{d})$ are mostly derived from freshwater sources and accounted for 38 percent of the total freshwater withdrawals and about 91 percent of total saline-water withdrawals. Irrigation withdrawals totaled 115,000 Mgal/d and accounted for 38 percent of total freshwater withdrawals. Total withdrawals for public supply $(42,000 \mathrm{Mgal} / \mathrm{d})$ represented nearly 14 percent of the total freshwater withdrawals.

In 2010, more than 50 percent of the total withdrawals in the United States were accounted for by 12 States: California, Texas, Idaho, Florida, Illinois, North Carolina, Arkansas, Colorado, Michigan, New York, Alabama, and Ohio. California accounted for 11 percent of the total withdrawals for all categories and 10 percent of total freshwater withdrawals for all categories nationwide. Texas accounted for about 7 percent of total withdrawals for all categories, predominantly for thermoelectric power, irrigation, and public supply. Florida had the largest saline withdrawals, accounting for 18 percent of the total in the United States, mostly saline surface-water withdrawals for thermoelectric power. Oklahoma and Texas accounted for about 70 percent of the total saline groundwater withdrawals in the United States, mostly for mining.

Water withdrawals by category and State are listed for surface water in tables $3 A$ and $3 B$ and for groundwater in tables $4 A$ and $4 B$. In 2010, more surface water than groundwater was withdrawn for all uses except domestic, livestock, and mining. Thermoelectric power accounted for 51 percent of the total fresh surface-water withdrawals and irrigation accounted for 29 percent. The largest surfacewater withdrawals in the Nation were in California, where irrigation accounted for 76 percent of total fresh surfacewater withdrawals. Large quantities of fresh surface water were also withdrawn for thermoelectric power in Illinois, Texas, Michigan, and Alabama. Large saline surface-water withdrawals for thermoelectric power occurred in Florida, California, Maryland, and New York, which cumulatively accounted for 57 percent of the national total saline surfacewater withdrawals.

Of the total fresh groundwater withdrawals (76,000 Mgal/d), irrigation accounted for 65 percent, primarily in California, Arkansas, Texas, and Nebraska. Fresh groundwater irrigation withdrawals in these four States cumulatively accounted for 42 percent of the national total fresh groundwater withdrawals. Nearly all groundwater withdrawals (96 percent) were from freshwater, and irrigation used greater than three times more fresh groundwater than public supply, which was the next largest use of groundwater in the Nation. The largest withdrawals of saline groundwater occurred in Oklahoma and Texas.

The geographic distribution of total withdrawals in the United States is shown in figure 2. The geographic distribution of total surface water and groundwater, and total freshwater and saline-water withdrawals by State is shown in figure 3. 
Public supply, 12 percent
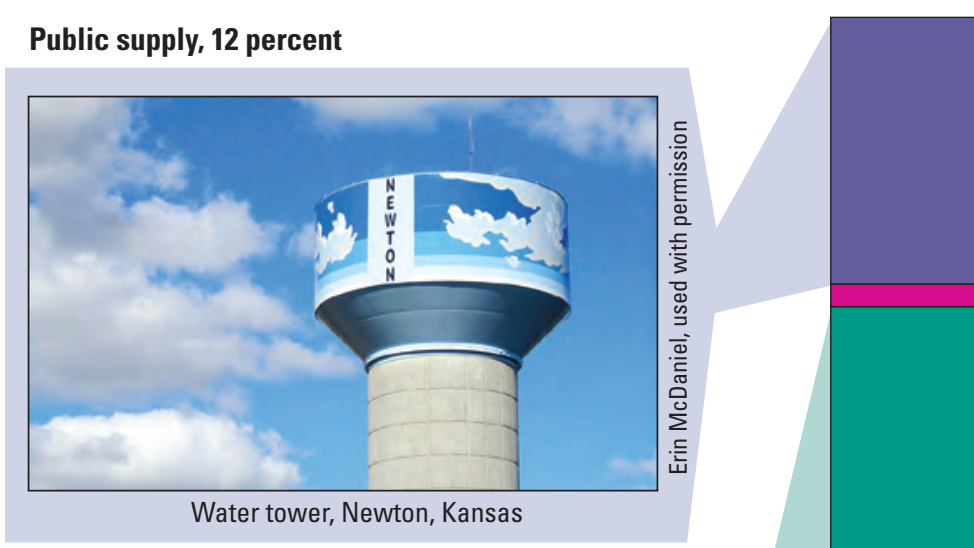

Domestic, 1 percent

\section{Irrigation, 33 percent}

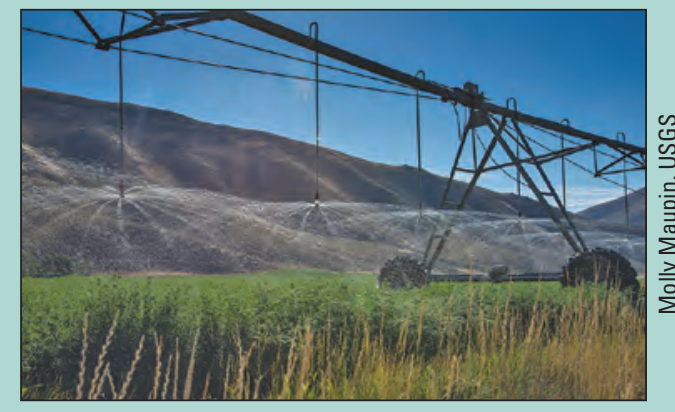

Sprinkler irrigation system, Blaine County, Idaho

\section{Aquaculture, 3 percent}

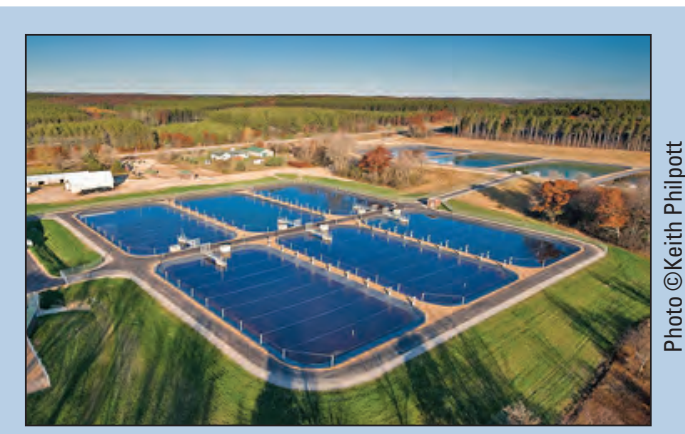

Wild Rose Fish Hatchery, Waushara County, Wisconsin

\section{Mining, 1 percent}

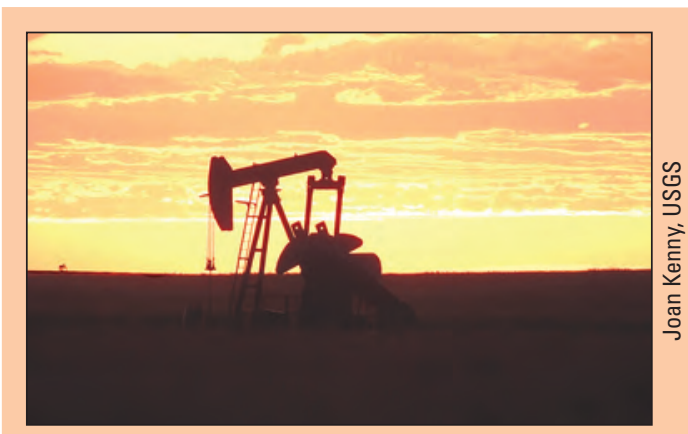

Pumpjack in Gove County, Kansas
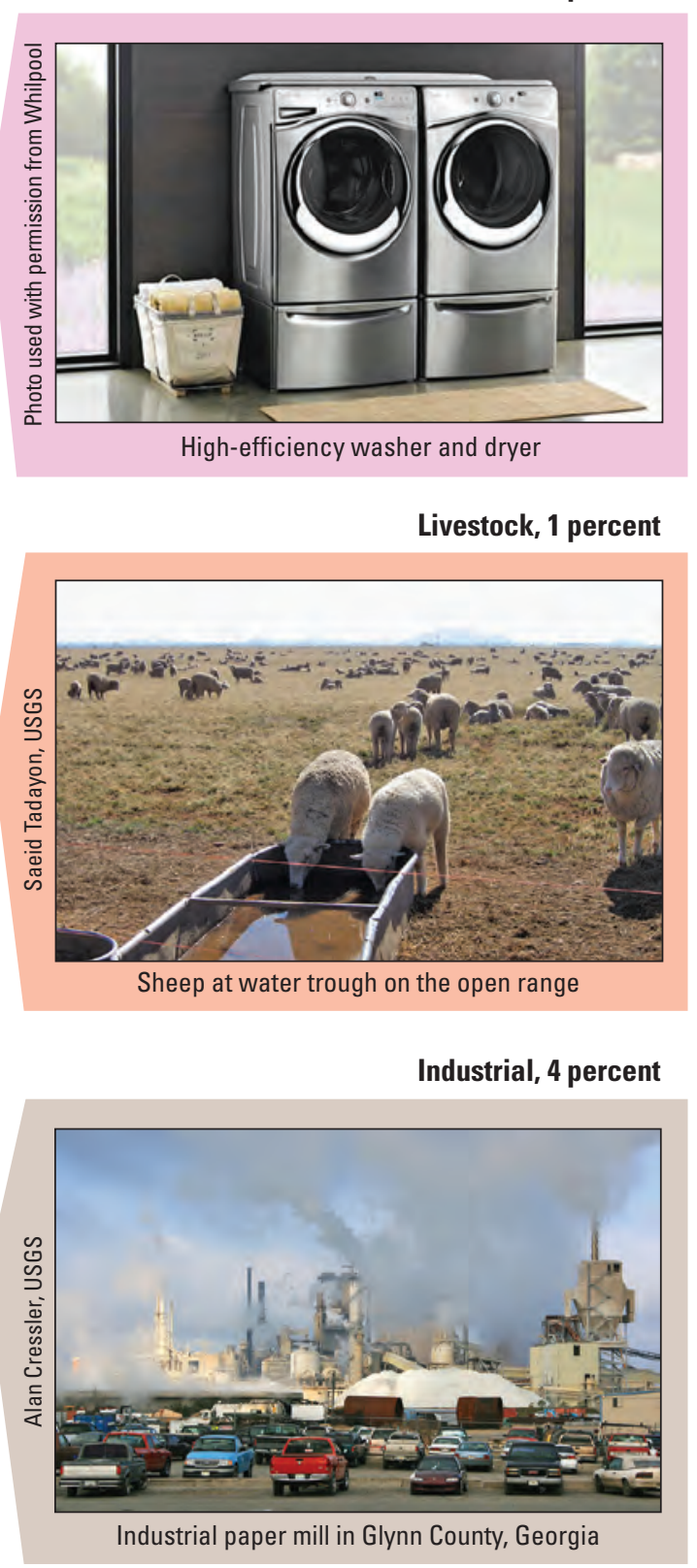

Thermoelectric power, 45 percent

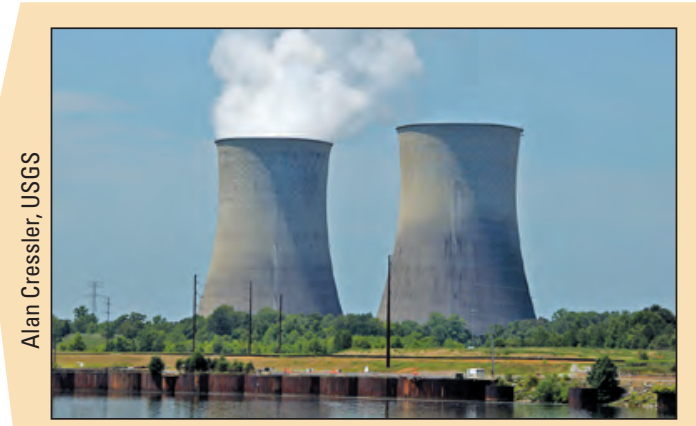

Watts Bar Nuclear Powerplant, Rhea County, Tennessee

Figure 1. Total water withdrawals by category, 2010. 
Table 1. Total water withdrawals by source and State, 2010.

[Values may not sum to totals because of independent rounding]

\begin{tabular}{|c|c|c|c|c|c|c|c|c|c|c|c|c|c|}
\hline \multirow{4}{*}{ State } & \multirow{4}{*}{$\begin{array}{l}\text { Population } \\
\text { (in } \\
\text { thousands) }\end{array}$} & \multicolumn{6}{|c|}{$\begin{array}{c}\text { Withdrawals } \\
\text { (in million gallons per day) }\end{array}$} & \multirow{3}{*}{\multicolumn{3}{|c|}{$\begin{array}{c}\text { Withdrawals } \\
\text { (in million gallons per day) } \\
\text { Total }\end{array}$}} & \multirow{3}{*}{\multicolumn{3}{|c|}{$\begin{array}{c}\begin{array}{c}\text { Withdrawals } \\
\text { (in thousand acre-feet per year) }\end{array} \\
\text { Total }\end{array}$}} \\
\hline & & \multirow{2}{*}{\multicolumn{6}{|c|}{ By source and type }} & & & & & & \\
\hline & & & \multicolumn{2}{|c|}{ Groundwater } & \multicolumn{2}{|c|}{ Surface water } & & & & & & & \\
\hline & & Fresh & Saline & Total & Fresh & Saline & Total & Fresh & Saline & Total & Fresh & Saline & Total \\
\hline Alabama ........................... & 4,780 & 494 & 0 & 494 & 9,470 & 0 & 9,470 & 9,960 & 0 & 9,960 & 11,200 & 0 & 11,200 \\
\hline Alaska .................................. & 710 & 478 & 144 & 622 & 391 & 80.7 & 472 & 869 & 225 & 1,090 & 975 & 252 & 1,230 \\
\hline Arizona........................... & 6,390 & 2,550 & 0 & 2,550 & 3,540 & 0 & 3,540 & 6,090 & 0 & 6,090 & 6,820 & 0 & 6,820 \\
\hline 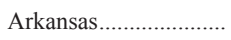 & 2,920 & 7,780 & 5.05 & 7,790 & 3,540 & 0 & 3,540 & 11,300 & 5.05 & 11,300 & 12,700 & 5.66 & 12,700 \\
\hline California ........................ & 37,300 & 12,300 & 369 & 12,700 & 18,800 & 6,490 & 25,300 & 31,100 & 6,860 & 38,000 & 34,900 & 7,690 & 42,600 \\
\hline 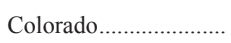 & 5,030 & 1,540 & 19.4 & 1,560 & 9,440 & 0 & 9,440 & 11,000 & 19.4 & 11,000 & 12,300 & 21.8 & 12,300 \\
\hline 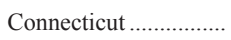 & 3,570 & 216 & 0 & 216 & 600 & 2,490 & 3,090 & 816 & 2,490 & 3,310 & 915 & 2,800 & 3,710 \\
\hline 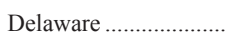 & 898 & 156 & 0 & 156 & 144 & 417 & 561 & 300 & 417 & 717 & 337 & 468 & 804 \\
\hline District of Columbia... & 602 & 0.05 & 0 & 0.05 & 0.05 & 0 & 0.05 & 0.10 & 0 & 0.10 & 0.11 & 0 & 0.11 \\
\hline Florida ............................. & 18,800 & 3,970 & 154 & 4,120 & 2,230 & 8,580 & 10,800 & 6,200 & 8,740 & 14,900 & 6,950 & 9,790 & 16,700 \\
\hline 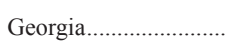 & 9,690 & 1,230 & 0 & 1,230 & 3,210 & 283 & 3,490 & 4,440 & 283 & 4,720 & 4,970 & 317 & 5,290 \\
\hline 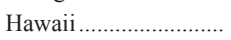 & 1,360 & 423 & 50.8 & 474 & 248 & 552 & 800 & 671 & 603 & 1,270 & 752 & 676 & 1,430 \\
\hline Idaho .................................... & 1,570 & 4,250 & 0 & 4,250 & 13,000 & 0 & 13,000 & 17,200 & 0 & 17,200 & 19,300 & 0 & 19,300 \\
\hline Illinois ............................... & 12,800 & 853 & 25.5 & 879 & 12,200 & 0 & 12,200 & 13,100 & 25.5 & 13,100 & 14,600 & 28.6 & 14,700 \\
\hline 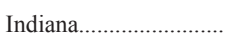 & 6,480 & 720 & 0 & 720 & 7,920 & 0 & 7,920 & 8,640 & 0 & 8,640 & 9,690 & 0 & 9,690 \\
\hline 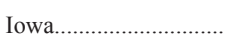 & 3,050 & 650 & 0 & 650 & 2,420 & 0 & 2,420 & 3,070 & 0 & 3,070 & 3,440 & 0 & 3,440 \\
\hline 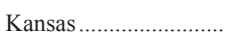 & 2,850 & 3,200 & 0 & 3,200 & 800 & 0 & 800 & 4,000 & 0 & 4,000 & 4,490 & 0 & 4,490 \\
\hline 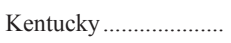 & 4,340 & 199 & 0 & 199 & 4,130 & 0 & 4,130 & 4,330 & 0 & 4,330 & 4,850 & 0 & 4,850 \\
\hline Louisiana........................ & 4,530 & 1,570 & 0 & 1,570 & 6,960 & 1.68 & 6,970 & 8,540 & 1.68 & 8,540 & 9,570 & 1.88 & 9,570 \\
\hline Maine .............................. & 1,330 & 99.4 & 0 & 99.4 & 309 & 40.8 & 350 & 408 & 40.8 & 449 & 458 & 45.8 & 504 \\
\hline 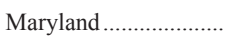 & 5,770 & 260 & 0 & 260 & 1,210 & 5,910 & 7,120 & 1,470 & 5,910 & 7,380 & 1,650 & 6,630 & 8,280 \\
\hline Massachusetts ................ & 6,550 & 361 & 0 & 361 & 703 & 1,930 & 2,640 & 1,060 & 1,930 & 3,000 & 1,190 & 2,170 & 3,360 \\
\hline Michigan ............................. & 9,880 & 693 & 0.57 & 694 & 10,100 & 0 & 10,100 & 10,800 & 0.57 & 10,800 & 12,100 & 0.64 & 12,100 \\
\hline Minnesota......................... & 5,300 & 736 & 0 & 736 & 3,080 & 0 & 3,080 & 3,820 & 0 & 3,820 & 4,280 & 0 & 4,280 \\
\hline Mississippi ...................... & 2,970 & 2,610 & 19.6 & 2,630 & 1,240 & 62.4 & 1,300 & 3,850 & 82.0 & 3,930 & 4,320 & 92.0 & 4,410 \\
\hline Missouri ... & 5,990 & 1,810 & 0 & 1,810 & 6,750 & 0 & 6,750 & 8,570 & 0 & 8,570 & 9,610 & 0 & 9,610 \\
\hline 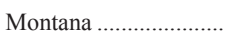 & 989 & 268 & 18.6 & 286 & 7,360 & 0 & 7,360 & 7,630 & 18.6 & 7,650 & 8,550 & 20.9 & 8,570 \\
\hline 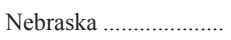 & 1,830 & 4,710 & 0.13 & 4,710 & 3,320 & 0 & 3,320 & 8,040 & 0.13 & 8,040 & 9,010 & 0.15 & 9,010 \\
\hline 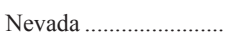 & 2,700 & 1,190 & 11.9 & 1,200 & 1,420 & 0 & 1,420 & 2,610 & 11.9 & 2,620 & 2,930 & 13.4 & 2,940 \\
\hline New Hampshire ........... & 1,320 & 89.7 & 0 & 89.7 & 277 & 848 & 1,120 & 367 & 848 & 1,210 & 411 & 951 & 1,360 \\
\hline New Jersey ....................... & 8,790 & 612 & 0 & 612 & 1,320 & 3,740 & 5,060 & 1,930 & 3,740 & 5,670 & 2,170 & 4,190 & 6,360 \\
\hline New Mexico..................... & 2,060 & 1,570 & 0 & 1,570 & 1,590 & 0 & 1,590 & 3,160 & 0 & 3,160 & 3,540 & 0 & 3,540 \\
\hline New York ............................... & 19,400 & 704 & 0 & 704 & 5,020 & 4,850 & 9,870 & 5,730 & 4,850 & 10,600 & 6,420 & 5,430 & 11,900 \\
\hline North Carolina ............. & 9,540 & 694 & 0 & 694 & 10,400 & 1,360 & 11,700 & 11,100 & 1,360 & 12,400 & 12,400 & 1,530 & 13,900 \\
\hline North Dakota.................... & 673 & 139 & 13.6 & 153 & 994 & 0 & 994 & 1,130 & 13.6 & 1,150 & 1,270 & 15.3 & 1,290 \\
\hline 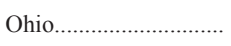 & 11,500 & 929 & 0 & 929 & 8,510 & 0 & 8,510 & 9,440 & 0 & 9,440 & 10,600 & 0 & 10,600 \\
\hline 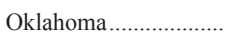 & 3,750 & 635 & 1,400 & 2,030 & 1,140 & 0 & 1,140 & 1,770 & 1,400 & 3,170 & 1,990 & 1,570 & 3,550 \\
\hline 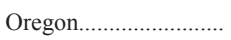 & 3,830 & 2,130 & 0 & 2,130 & 4,600 & 0 & 4,600 & 6,730 & 0 & 6,730 & 7,550 & 0 & 7,550 \\
\hline Pennsylvania .................. & 12,700 & 657 & 0 & 657 & 7,480 & 0 & 7,480 & 8,130 & 0 & 8,130 & 9,120 & 0 & 9,120 \\
\hline Rhode Island .................. & 1,050 & 36.5 & 0 & 36.5 & 98.0 & 241 & 339 & 134 & 241 & 376 & 151 & 270 & 421 \\
\hline South Carolina ............. & 4,630 & 339 & 0 & 339 & 6,440 & 0 & 6,440 & 6,780 & 0 & 6,780 & 7,600 & 0 & 7,600 \\
\hline South Dakota.................. & 814 & 339 & 0 & 339 & 287 & 0 & 287 & 626 & 0 & 626 & 701 & 0 & 701 \\
\hline Tennessee ........................ & 6,350 & 470 & 0 & 470 & 7,230 & 0 & 7,230 & 7,700 & 0 & 7,700 & 8,630 & 0 & 8,630 \\
\hline 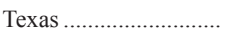 & 25,100 & 6,830 & 884 & 7,710 & 15,800 & 1,280 & 17,100 & 22,600 & 2,160 & 24,800 & 25,400 & 2,420 & 27,800 \\
\hline Utah & 2,760 & 1,030 & 92.6 & 1,120 & 3,110 & 238 & 3,340 & 4,130 & 331 & 4,460 & 4,630 & 371 & 5,000 \\
\hline 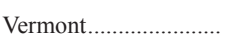 & 626 & 41.6 & 0 & 41.6 & 389 & 0 & 389 & 431 & 0 & 431 & 483 & 0 & 483 \\
\hline Virginia.............................. & 8,000 & 299 & 9.97 & 309 & 4,140 & 3,200 & 7,340 & 4,440 & 3,210 & 7,650 & 4,970 & 3,600 & 8,570 \\
\hline 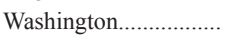 & 6,720 & 1,600 & 0 & 1,600 & 3,320 & 33.1 & 3,350 & 4,920 & 33.1 & 4,960 & 5,520 & 37.1 & 5,560 \\
\hline West Virginia................... & 1,850 & 121 & 4.82 & 125 & 3,410 & 0 & 3,410 & 3,530 & 4.82 & 3,530 & 3,960 & 5.40 & 3,960 \\
\hline Wisconsin ........................ & 5,690 & 754 & 0 & 754 & 5,400 & 0 & 5,400 & 6,160 & 0 & 6,160 & 6,900 & 0 & 6,900 \\
\hline 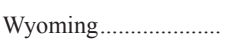 & 564 & 550 & 67.1 & 617 & 4,080 & 0 & 4,080 & 4,630 & 67.1 & 4,700 & 5,200 & 75.2 & 5,270 \\
\hline Puerto Rico..................... & 3,730 & 125 & 0.32 & 125 & 611 & 2,270 & 2,880 & 736 & 2,270 & 3,010 & 825 & 2,550 & 3,370 \\
\hline U.S. Virgin Islands.. & 106 & 1.14 & 0 & 1.14 & 2.85 & 124 & 127 & 3.99 & 124 & 128 & 4.47 & 139 & 143 \\
\hline TOTAL & 313,000 & 76,000 & 3,290 & 79,300 & 230,000 & 45,000 & 275,000 & 306,000 & 48,300 & 355,000 & 343,000 & 54,200 & 397,000 \\
\hline
\end{tabular}


Table 2A. Total water withdrawals by water-use category, 2010, in million gallons per day.

[Values may not sum to totals because of independent rounding]

\begin{tabular}{|c|c|c|c|c|c|c|c|c|c|c|c|c|c|c|}
\hline \multirow[t]{2}{*}{ State } & \multirow{2}{*}{$\begin{array}{l}\text { Public } \\
\text { supply }\end{array}$} & \multirow{2}{*}{$\begin{array}{c}\text { Self- } \\
\text { supplied } \\
\text { domestic }\end{array}$} & \multirow{2}{*}{$\begin{array}{l}\text { Irriga- } \\
\text { tion }\end{array}$} & \multirow{2}{*}{$\begin{array}{l}\text { Live- } \\
\text { stock }\end{array}$} & \multirow{2}{*}{$\begin{array}{l}\text { Aqua- } \\
\text { culture }\end{array}$} & \multicolumn{2}{|c|}{$\begin{array}{l}\text { Self-supplied } \\
\text { industrial }\end{array}$} & \multicolumn{2}{|c|}{ Mining } & \multicolumn{2}{|c|}{$\begin{array}{c}\text { Thermoelectric } \\
\text { power }\end{array}$} & \multicolumn{3}{|c|}{ Total } \\
\hline & & & & & & Fresh & Saline & Fresh & Saline & Fresh & Saline & Fresh & Saline & Total \\
\hline 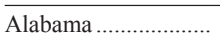 & 831 & 38.0 & 159 & 26.5 & 59.1 & 574 & 0 & 20.2 & 0 & 8,250 & 0 & 9,960 & 0 & 9,960 \\
\hline 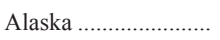 & 79.0 & 14.8 & 1.59 & 0.25 & 684 & 7.78 & 4.30 & 24.1 & 221 & 58.0 & 0 & 869 & 225 & 1,090 \\
\hline 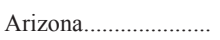 & 1,210 & 27.2 & 4,570 & 27.0 & 47.3 & 12.9 & 0 & 86.6 & 0 & 104 & 0 & 6,090 & 0 & 6,090 \\
\hline 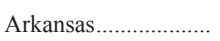 & 429 & 12.8 & 8,720 & 39.0 & 268 & 271 & 5.05 & 44.3 & 0 & 1,540 & 0 & 11,300 & 5.05 & 11,300 \\
\hline California ...................... & 6,300 & 172 & 23,100 & 188 & 973 & 400 & 0 & 36.4 & 236 & 65.4 & 6,540 & 31,100 & 6,860 & 38,000 \\
\hline Colorado & 848 & 37.9 & 9,710 & 36.9 & 122 & 130 & 0 & 8.51 & 19.4 & 77 & 0 & 11,000 & 19.4 & 11,000 \\
\hline 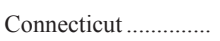 & 427 & 65.4 & 24.0 & 1.01 & 29.7 & 66.5 & 38.5 & 4.72 & 0 & 198 & 2,460 & 816 & 2,490 & 3,310 \\
\hline 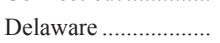 & 78.1 & 14.8 & 101 & 1.31 & 0.06 & 96.0 & 0 & 0.85 & 0 & 7.82 & 417 & 300 & 417 & 717 \\
\hline District of Columbia & 0 & 0 & 0.10 & 0 & 0 & 0 & 0 & 0 & 0 & 0 & 0 & 0.10 & 0 & 0.10 \\
\hline Florida .......................... & 2,270 & 214 & 2,920 & 21.3 & 1.86 & 213 & 0 & 113 & 0 & 613 & 8,570 & 6,200 & 8,740 & 14,900 \\
\hline 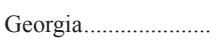 & 1,120 & 115 & 839 & 29.3 & 49.8 & 487 & 0 & 27.7 & 0 & 1,770 & 283 & 4,440 & 283 & 4,720 \\
\hline Hawaii .............................. & 274 & 8.02 & 323 & 1.83 & 4.54 & 4.63 & 0 & 1.51 & 0 & 53.2 & 603 & 671 & 603 & 1,270 \\
\hline Idaho ................................... & 239 & 79.0 & 14,000 & 47.5 & 2,750 & 49.7 & 0 & 20.2 & 0 & 0.88 & 0 & 17,200 & 0 & 17,200 \\
\hline 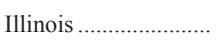 & 1,500 & 92.4 & 226 & 36.1 & 32.0 & 390 & 0 & 70.9 & 25.5 & 10,700 & 0 & 13,100 & 25.5 & 13,100 \\
\hline 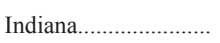 & 656 & 126 & 137 & 39.2 & 8.57 & 2,210 & 0 & 88.2 & 0 & 5,380 & 0 & 8,640 & 0 & 8,640 \\
\hline Iowa................................... & 393 & 38.4 & 42.8 & 136 & 18.9 & 125 & 0 & 79.6 & 0 & 2,240 & 0 & 3,070 & 0 & 3,070 \\
\hline 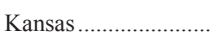 & 391 & 14.9 & 3,040 & 114 & 12.9 & 40.3 & 0 & 13.3 & 0 & 377 & 0 & 4,000 & 0 & 4,000 \\
\hline Kentucky ……............ & 572 & 33.2 & 29.0 & 43.8 & 34.1 & 228 & 0 & 30.8 & 0 & 3,360 & 0 & 4,330 & 0 & 4,330 \\
\hline Louisiana ...................... & 746 & 47.0 & 928 & 8.03 & 311 & 2,060 & 0 & 11.3 & 0 & 4,430 & 1.68 & 8,540 & 1.68 & 8,540 \\
\hline Maine ……………............ & 91.3 & 33.0 & 11.3 & 2.29 & 46.9 & 192 & 14.8 & 4.87 & 0 & 26.8 & 26.0 & 408 & 40.8 & 449 \\
\hline 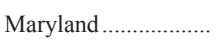 & 790 & 85.6 & 72.1 & 8.25 & 20.8 & 50.0 & 146 & 9.43 & 0 & 436 & 5,760 & 1,470 & 5,910 & 7,380 \\
\hline Massachusetts ........... & 679 & 37.9 & 139 & 1.40 & 49.6 & 16.3 & 0 & 6.60 & 0 & 134 & 1,930 & 1,060 & 1,930 & 3,000 \\
\hline 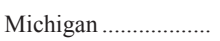 & 1,090 & 231 & 209 & 19.6 & 82.7 & 612 & 0 & 76.2 & 0.57 & 8,520 & 0 & 10,800 & 0.57 & 10,800 \\
\hline 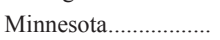 & 542 & 79.0 & 197 & 59.3 & 16.9 & 134 & 0 & 285 & 0 & 2,510 & 0 & 3,820 & 0 & 3,820 \\
\hline Mississippi .................... & 395 & 44.6 & 2,090 & 18.4 & 133 & 203 & 0 & 8.78 & 12.6 & 956 & 69.5 & 3,850 & 82.0 & 3,930 \\
\hline 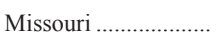 & 836 & 61.8 & 1,400 & 72.9 & 181 & 68.4 & 0 & 32.9 & 0 & 5,910 & 0 & 8,570 & 0 & 8,570 \\
\hline Montana .......................... & 138 & 22.2 & 7,160 & 41.8 & 18.9 & 66.4 & 0 & 27.9 & 18.6 & 151 & 0 & 7,630 & 18.6 & 7,650 \\
\hline Nebraska ....................... & 296 & 44.0 & 5,660 & 114 & 88.3 & 31.1 & 0 & 8.86 & 0.13 & 1,790 & 0 & 8,040 & 0.13 & 8,040 \\
\hline Nevada .......................... & 581 & 29.8 & 1,570 & 5.06 & 49.5 & 5.23 & 0 & 345 & 0.95 & 21.6 & 11.0 & 2,610 & 11.9 & 2,620 \\
\hline New Hampshire ........ & 91.2 & 33.3 & 1.92 & 0.89 & 16.6 & 17.7 & 0 & 2.85 & 0 & 202 & 848 & 367 & 848 & 1,210 \\
\hline New Jersey ................. & 1,080 & 98.3 & 138 & 0.98 & 9.16 & 83.3 & 0 & 8.64 & 0 & 513 & 3,740 & 1,930 & 3,740 & 5,670 \\
\hline New Mexico..................... & 283 & 25.8 & 2,700 & 35.8 & 20.1 & 11.1 & 0 & 37.1 & 0 & 51.9 & 0 & 3,160 & 0 & 3,160 \\
\hline 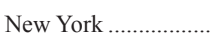 & 2,260 & 152 & 70.4 & 22.6 & 40.2 & 352 & 0 & 72.4 & 0 & 2,760 & 4,850 & 5,730 & 4,850 & 10,600 \\
\hline North Carolina .......... & 960 & 231 & 367 & 72.0 & 1,470 & 271 & 0 & 32.6 & 0 & 7,660 & 1,360 & 11,100 & 1,360 & 12,400 \\
\hline North Dakota.................. & 68.8 & 3.68 & 165 & 21.6 & 5.92 & 18.7 & 0 & 13.4 & 13.6 & 837 & 0 & 1,130 & 13.6 & 1,150 \\
\hline Ohio..................................... & 1,370 & 137 & 52.6 & 24.0 & 34.3 & 489 & 0 & 115 & 0 & 7,220 & 0 & 9,440 & 0 & 9,440 \\
\hline 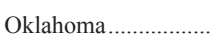 & 657 & 26.8 & 564 & 88.8 & 10.7 & 20.8 & 0 & 18.0 & 1,400 & 385 & 0 & 1,770 & 1,400 & 3,170 \\
\hline Oregon............................ & 534 & 67.1 & 5,260 & 17.0 & 712 & 126 & 0 & 8.64 & 0 & 12.7 & 0 & 6,730 & 0 & 6,730 \\
\hline Pennsylvania .............. & 1,420 & 201 & 27.1 & 52.3 & 108 & 866 & 0 & 62.0 & 0 & 5,390 & 0 & 8,130 & 0 & 8,130 \\
\hline 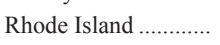 & 108 & 8.02 & 2.69 & 0.18 & 14.5 & 7.52 & 0 & 0.92 & 0 & 1.44 & 232 & 135 & 241 & 376 \\
\hline South Carolina .......... & 619 & 115 & 125 & 12.0 & 11.0 & 388 & 0 & 8.43 & 0 & 5,500 & 0 & 6,780 & 0 & 6,780 \\
\hline South Dakota.................. & 124 & 5.37 & 362 & 47.4 & 48.4 & 9.48 & 0 & 18.2 & 0 & 10.3 & 0 & 626 & 0 & 626 \\
\hline Tennessee ................... & 918 & 38.7 & 71.9 & 27.5 & 52.6 & 776 & 0 & 14.6 & 0 & 5,800 & 0 & 7,700 & 0 & 7,700 \\
\hline 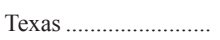 & 3,990 & 259 & 6,830 & 259 & 31.4 & 680 & 610 & 203 & 810 & 10,500 & 661 & 22,600 & 2,160 & 24,800 \\
\hline 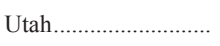 & 673 & 8.44 & 3,220 & 16.5 & 97.1 & 47.6 & 70.6 & 4.19 & 246 & 69.6 & 11.0 & 4,130 & 331 & 4,460 \\
\hline Vermont............................ & 43.1 & 13.6 & 2.45 & 5.63 & 10.9 & 5.69 & 0 & 3.85 & 0 & 345 & 0 & 431 & 0 & 431 \\
\hline Virginia........................... & 665 & 124 & 61.4 & 27.4 & 295 & 383 & 56.1 & 34.9 & 0 & 2,860 & 3,150 & 4,440 & 3,210 & 7,650 \\
\hline 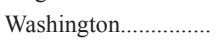 & 910 & 113 & 3,150 & 27.8 & 213 & 458 & 33.1 & 16.7 & 0 & 37.9 & 0 & 4,920 & 33.1 & 4,960 \\
\hline West Virginia.................. & 189 & 31.5 & 0.09 & 5.08 & 52.3 & 764 & 3.80 & 14.5 & 1.02 & 2,470 & 0 & 3,530 & 4.82 & 3,530 \\
\hline Wisconsin ......................... & 481 & 78.4 & 379 & 73.1 & 55.8 & 436 & 0 & 19.6 & 0 & 4,630 & 0 & 6,160 & 0 & 6,160 \\
\hline 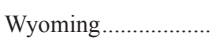 & 99.0 & 8.55 & 4,370 & 16.5 & 20.8 & 6.74 & 0 & 50.1 & 67.1 & 63.4 & 0 & 4,630 & 67.1 & 4,700 \\
\hline Puerto Rico....................... & 677 & 2.41 & 38.2 & 7.81 & 0.41 & 4.30 & 0 & 1.61 & 0.32 & 3.78 & 2,270 & 736 & 2,270 & 3,010 \\
\hline U.S. Virgin Islands... & 5.86 & 2.67 & 0 & 0.02 & 0 & 0.22 & 2.62 & 0 & 0.04 & 0.17 & 116 & 3.99 & 124 & 128 \\
\hline TOTAL & 42,000 & 3,600 & 5,000 & 2,000 & 9,420 & 15,000 & 986 & 2,250 & 3,070 & 17,000 & 43,900 & 306,000 & 48,300 & 355,000 \\
\hline
\end{tabular}


Table 2B. Total water withdrawals by water-use category, 2010 , in thousand acre-feet per year.

[Values may not sum to totals because of independent rounding]

\begin{tabular}{|c|c|c|c|c|c|c|c|c|c|c|c|c|c|c|}
\hline \multirow[t]{2}{*}{ State } & \multirow{2}{*}{$\begin{array}{l}\text { Public } \\
\text { supply }\end{array}$} & \multirow{2}{*}{$\begin{array}{c}\text { Self- } \\
\text { supplied } \\
\text { domestic }\end{array}$} & \multirow{2}{*}{$\begin{array}{l}\text { Irriga- } \\
\text { tion }\end{array}$} & \multirow{2}{*}{$\begin{array}{l}\text { Live- } \\
\text { stock }\end{array}$} & \multirow{2}{*}{$\begin{array}{l}\text { Aqua- } \\
\text { culture }\end{array}$} & \multicolumn{2}{|c|}{$\begin{array}{l}\text { Self-supplied } \\
\text { industrial }\end{array}$} & \multicolumn{2}{|c|}{ Mining } & \multicolumn{2}{|c|}{$\begin{array}{c}\text { Thermoelectric } \\
\text { power }\end{array}$} & \multicolumn{3}{|c|}{ Total } \\
\hline & & & & & & Fresh & Saline & Fresh & Saline & Fresh & Saline & Fresh & Saline & Total \\
\hline Alabama ............................ & 932 & 42.6 & 178 & 29.7 & 66.3 & 644 & 0 & 22.7 & 0 & 9,250 & 0 & 11,200 & 0 & 11,200 \\
\hline Alaska ......................... & 88.6 & 16.6 & 1.78 & 0.28 & 767 & 8.72 & 4.82 & 27.0 & 248 & 65.0 & 0 & 975 & 252 & 1,230 \\
\hline Arizona............................. & 1,360 & 30.5 & 5,120 & 30.2 & 53 & 14.5 & 0 & 97.1 & 0 & 117 & 0 & 6,820 & 0 & 6,820 \\
\hline 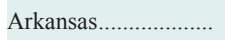 & 481 & 14.4 & 9,770 & 43.7 & 300 & 303 & 5.66 & 49.6 & 0 & 1,730 & 0 & 12,700 & 5.66 & 12,700 \\
\hline 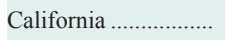 & 7,060 & 193 & 25,800 & 211 & 1,090 & 449 & 0 & 40.8 & 265 & 73.3 & 7,330 & 34,900 & 7,690 & 42,600 \\
\hline 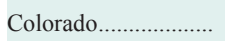 & 950 & 42.5 & 10,900 & 41.3 & 137 & 146 & 0 & 9.54 & 21.8 & 86.3 & 0 & 12,300 & 21.8 & 12,300 \\
\hline Connecticut ................ & 479 & 73.3 & 26.9 & 1.13 & 33.3 & 74.5 & 43.1 & 5.29 & 0 & 222 & 2,750 & 915 & 2,800 & 3,710 \\
\hline 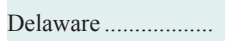 & 87.5 & 16.6 & 114 & 1.47 & 0.07 & 108 & 0 & 0.95 & 0 & 8.77 & 468 & 337 & 468 & 804 \\
\hline District of Columbia & 0 & 0 & 0.11 & 0 & 0 & 0 & 0 & 0 & 0 & 0 & 0 & 0.11 & 0 & 0.11 \\
\hline Florida …........................... & 2,540 & 240 & 3,270 & 23.9 & 2.09 & 239 & 0 & 127 & 0 & 687 & 9,610 & 6,950 & 9,790 & 16,700 \\
\hline 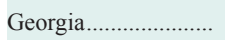 & 1,250 & 129 & 940 & 32.8 & 55.8 & 546 & 0 & 31.0 & 0 & 1,990 & 317 & 4,970 & 317 & 5,290 \\
\hline 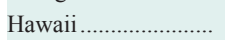 & 307 & 8.99 & 363 & 2.05 & 5.09 & 5.19 & 0 & 1.69 & 0 & 59.6 & 676 & 752 & 676 & 1,430 \\
\hline Idaho ................................... & 267 & 88.6 & 15,700 & 53.3 & 3,090 & 55.7 & 0 & 22.6 & 0 & 0.99 & 0 & 19,300 & 0 & 19,300 \\
\hline Illinois ............................... & 1,690 & 104 & 253 & 40.4 & 35.8 & 438 & 0 & 79.4 & 28.6 & 12,000 & 0 & 14,600 & 28.6 & 14,700 \\
\hline 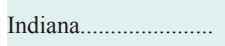 & 735 & 141 & 154 & 43.9 & 9.61 & 2,470 & 0 & 98.9 & 0 & 6,030 & 0 & 9,690 & 0 & 9,690 \\
\hline 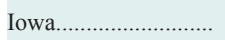 & 440 & 43.0 & 48.0 & 152 & 21.2 & 140 & 0 & 89.2 & 0 & 2,510 & 0 & 3,440 & 0 & 3,440 \\
\hline 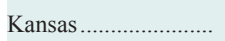 & 439 & 16.7 & 3,410 & 128 & 14.5 & 45.2 & 0 & 14.9 & 0 & 423 & 0 & 4,490 & 0 & 4,490 \\
\hline Kentucky ..................... & 641 & 37.2 & 32.5 & 49.1 & 38.2 & 255 & 0 & 34.5 & 0 & 3,760 & 0 & 4,850 & 0 & 4,850 \\
\hline 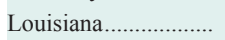 & 837 & 52.7 & 1,040 & 9.00 & 349 & 2,310 & 0 & 12.6 & 0 & 4,960 & 1.88 & 9,570 & 1.88 & 9,570 \\
\hline Maine …............................... & 102 & 37.0 & 12.6 & 2.57 & 52.6 & 215 & 16.6 & 5.46 & 0 & 30.1 & 29.1 & 458 & 45.8 & 504 \\
\hline 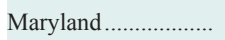 & 885 & 95.9 & 80.8 & 9.25 & 23.3 & 56.0 & 164 & 10.6 & 0 & 489 & 6,460 & 1,650 & 6,630 & 8,280 \\
\hline Massachusetts ........... & 761 & 42.5 & 156 & 1.57 & 55.7 & 18.3 & 0 & 7.40 & 0 & 151 & 2,160 & 1,190 & 2,170 & 3,360 \\
\hline Michigan ........................ & 1,220 & 259 & 235 & 22.0 & 92.7 & 686 & 0 & 85.4 & 0.64 & 9,550 & 0 & 12,100 & 0.64 & 12,100 \\
\hline Minnesota......................... & 607 & 88.5 & 221 & 66.5 & 18.9 & 150 & 0 & 319 & 0 & 2,810 & 0 & 4,280 & 0 & 4,280 \\
\hline Mississippi .................... & 443 & 50.0 & 2,350 & 20.6 & 149 & 227 & 0 & 9.84 & 14.1 & 1,070 & 77.9 & 4,320 & 92.0 & 4,410 \\
\hline Missouri ......................... & 938 & 69.3 & 1,570 & 81.7 & 202 & 76.7 & 0 & 36.8 & 0 & 6,630 & 0 & 9,610 & 0 & 9,610 \\
\hline Montana .......................... & 155 & 24.9 & 8,030 & 46.9 & 21.2 & 74.5 & 0 & 31.3 & 20.9 & 169 & 0 & 8,550 & 20.9 & 8,570 \\
\hline Nebraska ..................... & 331 & 49.3 & 6,340 & 128 & 99.0 & 34.9 & 0 & 9.93 & 0.15 & 2,010 & 0 & 9,010 & 0.15 & 9,010 \\
\hline 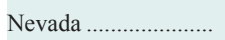 & 651 & 33.4 & 1,760 & 5.67 & 55.4 & 5.86 & 0 & 387 & 1.06 & 24.2 & 12.3 & 2,930 & 13.4 & 2,940 \\
\hline New Hampshire ........ & 102 & 37.4 & 2.15 & 1.00 & 18.6 & 19.8 & 0 & 3.19 & 0 & 227 & 951 & 411 & 951 & 1,360 \\
\hline New Jersey .................. & 1,210 & 110 & 154 & 1.10 & 10.3 & 93.3 & 0 & 9.69 & 0 & 575 & 4,190 & 2,170 & 4,190 & 6,360 \\
\hline New Mexico................. & 318 & 28.9 & 3,020 & 40.1 & 22.5 & 12.4 & 0 & 41.6 & 0 & 58.1 & 0 & 3,540 & 0 & 3,540 \\
\hline New York ...................... & 2,540 & 171 & 78.9 & 25.3 & 45.0 & 395 & 0 & 81.1 & 0 & 3,090 & 5,430 & 6,420 & 5,430 & 11,900 \\
\hline North Carolina .......... & 1,080 & 259 & 411 & 80.7 & 1,640 & 304 & 0 & 36.6 & 0 & 8,580 & 1,530 & 12,400 & 1,530 & 13,900 \\
\hline North Dakota................. & 77.2 & 4.13 & 185 & 24.2 & 6.64 & 20.9 & 0 & 15.0 & 15.3 & 938 & 0 & 1,270 & 15.3 & 1,290 \\
\hline 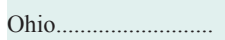 & 1,540 & 154 & 58.9 & 26.9 & 38.5 & 548 & 0 & 129 & 0 & 8,090 & 0 & 10,600 & 0 & 10,600 \\
\hline 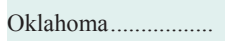 & 737 & 30.1 & 632 & 99.6 & 12.0 & 23.3 & 0 & 20.2 & 1,570 & 432 & 0 & 1,990 & 1,570 & 3,550 \\
\hline Oregon & 598 & 75.2 & 5,890 & 19.1 & 798 & 141 & 0 & 9.69 & 0 & 14.2 & 0 & 7550 & 0 & 7,550 \\
\hline Pennsylvania ................ & 1,600 & 225 & 30.4 & 58.6 & 121 & 971 & 0 & 69.5 & 0 & 6,050 & 0 & 9,120 & 0 & 9,120 \\
\hline Rhode Island ................ & 121 & 8.99 & 3.02 & 0.20 & 16.3 & 8.43 & 0 & 1.03 & 0 & 1.61 & 260 & 151 & 270 & 421 \\
\hline South Carolina ........... & 693 & 129 & 140 & 13.5 & 12.3 & 435 & 0 & 9.45 & 0 & 6,170 & 0 & 7,600 & 0 & 7,600 \\
\hline South Dakota................. & 139 & 6.02 & 406 & 53.1 & 54.3 & 10.6 & 0 & 20.4 & 0 & 11.5 & 0 & 701 & 0 & 701 \\
\hline Tennessee ..................... & 1,030 & 43.4 & 80.6 & 30.8 & 59.0 & 870 & 0 & 16.4 & 0 & 6,500 & 0 & 8,630 & 0 & 8,630 \\
\hline Texas .............................. & 4,480 & 290 & 7,660 & 290 & 35.2 & 762 & 684 & 227 & 908 & 11,700 & 741 & 25,400 & 2,420 & 27,800 \\
\hline 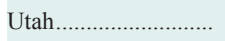 & 754 & 9.46 & 3,610 & 18.5 & 109 & 53.3 & 79.2 & 4.70 & 276 & 78.0 & 12.3 & 4,630 & 371 & 5,000 \\
\hline 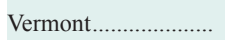 & 48.3 & 15.3 & 2.75 & 6.31 & 12.3 & 6.38 & 0 & 4.32 & 0 & 387 & 0 & 483 & 0 & 483 \\
\hline Virginia.............................. & 745 & 139 & 68.8 & 30.7 & 331 & 429 & 62.9 & 39.1 & 0 & 3,200 & 3,530 & 4,970 & 3,600 & 8,570 \\
\hline 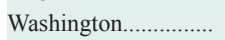 & 1,020 & 126 & 3,530 & 31.1 & 239 & 513 & 37.1 & 18.7 & 0 & 42.5 & 0 & 5,520 & 37.1 & 5,560 \\
\hline West Virginia................... & 212 & 35.3 & 0.10 & 5.69 & 58.7 & 857 & 4.26 & 16.3 & 1.14 & 2,770 & 0 & 3,960 & 5.40 & 3,960 \\
\hline Wisconsin ...................... & 540 & 87.8 & 425 & 81.9 & 62.5 & 489 & 0 & 21.9 & 0 & 5,200 & 0 & 6,900 & 0 & 6,900 \\
\hline Wyoming ......................... & 111 & 9.58 & 4,900 & 18.5 & 23.4 & 7.56 & 0 & 56.2 & 75.2 & 71.0 & 0 & 5,200 & 75.2 & 5,270 \\
\hline Puerto Rico................... & 759 & 2.70 & 42.8 & 8.76 & 0.46 & 4.82 & 0 & 1.80 & 0.36 & 4.24 & 2,540 & 825 & 2,550 & 3,370 \\
\hline U.S. Virgin Islands ... & 6.57 & 2.99 & 0 & 0.02 & 0 & 0.25 & 2.94 & 0 & 0.04 & 0.19 & 130 & 4.47 & 139 & 143 \\
\hline TOTAL & 47,100 & 4,040 & 129,000 & 2,240 & 10,600 & 16,800 & 1,100 & 2,520 & 3,440 & 131,000 & 49,200 & 343,000 & 54,200 & 397,000 \\
\hline
\end{tabular}


Table 3A. Surface-water withdrawals by water-use category, 2010, in million gallons per day.

[Values may not sum to totals because of independent rounding]

\begin{tabular}{|c|c|c|c|c|c|c|c|c|c|c|c|c|c|c|}
\hline \multirow[t]{2}{*}{ State } & \multirow{2}{*}{$\begin{array}{l}\text { Public } \\
\text { supply }\end{array}$} & \multirow{2}{*}{$\begin{array}{c}\text { Self- } \\
\text { supplied } \\
\text { domestic }\end{array}$} & \multirow{2}{*}{ Irrigation } & \multirow{2}{*}{$\begin{array}{l}\text { Live- } \\
\text { stock }\end{array}$} & \multirow{2}{*}{$\begin{array}{l}\text { Aqua- } \\
\text { culture }\end{array}$} & \multicolumn{2}{|c|}{$\begin{array}{l}\text { Self-supplied } \\
\text { industrial }\end{array}$} & \multicolumn{2}{|c|}{ Mining } & \multicolumn{2}{|c|}{$\begin{array}{c}\text { Thermoelectric } \\
\text { power }\end{array}$} & \multicolumn{3}{|c|}{ Total } \\
\hline & & & & & & Fresh & Saline & Fresh & Saline & Fresh & Saline & Fresh & Saline & Total \\
\hline Alabama .................... & 551 & 0 & 74.0 & 14.8 & 26.6 & 540 & 0 & 7.49 & 0 & 8,250 & 0 & 9,470 & 0 & 9,470 \\
\hline Alaska ............................ & 51.8 & 0.66 & 0.02 & 0.15 & 255 & 4.40 & 4.30 & 24.1 & 76.4 & 55.8 & 0 & 391 & 80.7 & 472 \\
\hline 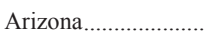 & 628 & 0 & 2,880 & 0 & 7.77 & 0 & 0 & 0 & 0 & 27.1 & 0 & 3,540 & 0 & 3,540 \\
\hline 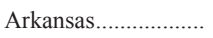 & 295 & 0 & 1,340 & 23.4 & 86.5 & 214 & 0 & 44.1 & 0 & 1,540 & 0 & 3,540 & 0 & 3,540 \\
\hline California ..................... & 3,470 & 29.4 & 14,400 & 103 & 802 & 1.13 & 0 & 12.2 & 0.05 & 32.2 & 6,490 & 18,800 & 6,490 & 25,300 \\
\hline 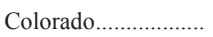 & 717 & 0 & 8,420 & 11.8 & 99.0 & 127 & 0 & 3.05 & 0 & 60.2 & 0 & 9,440 & 0 & 9,440 \\
\hline Connecticut ................. & 292 & 0 & 23.1 & 0 & 23.0 & 60.2 & 38.5 & 3.80 & 0 & 198 & 2,460 & 600 & 2,490 & 3,090 \\
\hline 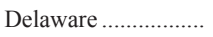 & 33.3 & 0 & 15.2 & 0 & 0 & 87.5 & 0 & 0.41 & 0 & 7.45 & 417 & 144 & 417 & 561 \\
\hline District of Columbia & 0 & 0 & 0.05 & 0 & 0 & 0 & 0 & 0 & 0 & 0 & 0 & 0.05 & 0 & 0.05 \\
\hline 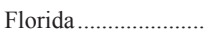 & 256 & 0 & 1,340 & 2.22 & 0 & 47.7 & 0 & 34.1 & 0 & 570 & 8,570 & 2,230 & 8,580 & 10,800 \\
\hline 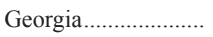 & 873 & 0 & 202 & 26.9 & 45.9 & 281 & 0 & 8.41 & 0 & 1,770 & 283 & 3,210 & 283 & 3,490 \\
\hline Hawaii ......................... & 15.8 & 6.17 & 223 & 1.20 & 2.40 & 0 & 0 & 0.11 & 0 & 0 & 552 & 248 & 552 & 800 \\
\hline 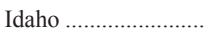 & 27.1 & 0 & 10,200 & 9.01 & 2,690 & 17.2 & 0 & 18.9 & 0 & 0 & 0 & 13,000 & 0 & 13,000 \\
\hline Illinois ....................... & 1,140 & 0 & 17.5 & 0.03 & 27.2 & 267 & 0 & 55.4 & 0 & 10,700 & 0 & 12,200 & 0 & 12,200 \\
\hline 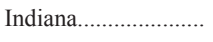 & 304 & 0 & 38.7 & 13.0 & 1.97 & 2,120 & 0 & 83.7 & 0 & 5,360 & 0 & 7,920 & 0 & 7,920 \\
\hline Iowa.................................... & 84.3 & 0 & 1.18 & 33.8 & 4.45 & 2.70 & 0 & 78.1 & 0 & 2,220 & 0 & 2,420 & 0 & 2,420 \\
\hline 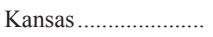 & 231 & 0 & 160 & 23.0 & 8.57 & 6.79 & 0 & 3.98 & 0 & 366 & 0 & 800 & 0 & 800 \\
\hline Kentucky .................... & 501 & 13.5 & 27.4 & 41.6 & 33.5 & 146 & 0 & 23.0 & 0 & 3,340 & 0 & 4,130 & 0 & 4,130 \\
\hline 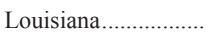 & 368 & 0 & 258 & 3.88 & 114 & 1,830 & 0 & 5.94 & 0 & 4,390 & 1.68 & 6,960 & 1.68 & 6,970 \\
\hline Maine ........................... & 63.6 & 0 & 8.77 & 0.58 & 21.2 & 185 & 14.8 & 3.73 & 0 & 25.9 & 26.0 & 309 & 40.8 & 350 \\
\hline 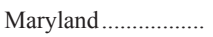 & 701 & 0 & 18.6 & 2.23 & 15.7 & 38.6 & 146 & 2.18 & 0 & 434 & 5,760 & 1,210 & 5,910 & 7,120 \\
\hline Massachusetts .......... & 489 & 0 & 21.4 & 0.50 & 42.4 & 12.1 & 0 & 4.78 & 0 & 134 & 1,930 & 703 & 1,930 & 2,640 \\
\hline Michigan .................... & 883 & 0 & 62.6 & 1.90 & 78.5 & 537 & 0 & 66.0 & 0 & 8,510 & 0 & 10,100 & 0 & 10,100 \\
\hline Minnesota.................... & 188 & 0 & 26.7 & 0 & 15.2 & 71.7 & 0 & 276 & 0 & 2,510 & 0 & 3,080 & 0 & 3,080 \\
\hline Mississippi ................ & 46.3 & 0 & 133 & 11.1 & 19.3 & 125 & 0 & 0.55 & 0 & 905 & 62.4 & 1,240 & 62.4 & 1,300 \\
\hline 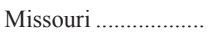 & 543 & 0 & 49.6 & 54.4 & 170 & 34.1 & 0 & 8.41 & 0 & 5,890 & 0 & 6,750 & 0 & 6,750 \\
\hline Montana ...................... & 72.4 & 1.04 & 7,030 & 29.5 & 16.4 & 29.6 & 0 & 26.2 & 0 & 150 & 0 & 7,360 & 0 & 7,360 \\
\hline Nebraska ..................... & 61.4 & 0 & 1,360 & 21.2 & 82.2 & 2.33 & 0 & 8.77 & 0 & 1,790 & 0 & 3,320 & 0 & 3,320 \\
\hline Nevada ........................ & 448 & 0 & 921 & 0 & 38.8 & 4.53 & 0 & 4.11 & 0 & 3.68 & 0 & 1,420 & 0 & 1,420 \\
\hline New Hampshire ....... & 56.6 & 0 & 0.67 & 0.22 & 8.48 & 7.06 & 0 & 2.84 & 0 & 201 & 848 & 277 & 848 & 1,120 \\
\hline New Jersey ................... & 682 & 0 & 70.1 & 0 & 0 & 48.5 & 0 & 6.91 & 0 & 512 & 3,740 & 1,320 & 3,740 & 5,060 \\
\hline New Mexico.............. & 72.4 & 0 & 1,460 & 3.03 & 4.32 & 0.83 & 0 & 9.68 & 0 & 42.3 & 0 & 1,590 & 0 & 1,590 \\
\hline New York .................... & 1,810 & 0 & 40.2 & 8.00 & 36.8 & 316 & 0 & 64.0 & 0 & 2,750 & 4,850 & 5,020 & 4,850 & 9,870 \\
\hline North Carolina ......... & 766 & 0 & 279 & 15.0 & 1,450 & 188 & 0 & 4.87 & 0 & 7,660 & 1,360 & 10,400 & 1,360 & 11,700 \\
\hline North Dakota............ & 38.3 & 0 & 87.2 & 8.62 & 5.92 & 12.9 & 0 & 4.63 & 0 & 837 & 0 & 994 & 0 & 994 \\
\hline Ohio.............................. & 918 & 2.75 & 35.4 & 16.3 & 19.0 & 293 & 0 & 35.8 & 0 & 7,190 & 0 & 8,510 & 0 & 8,510 \\
\hline Oklahoma .................... & 527 & 0 & 135 & 56.3 & 7.43 & 14.3 & 0 & 13.3 & 0 & 384 & 0 & 1,140 & 0 & 1,140 \\
\hline Oregon....................... & 420 & 7.07 & 3,350 & 14.0 & 679 & 123 & 0 & 1.17 & 0 & 11.2 & 0 & 4,600 & 0 & 4,600 \\
\hline Pennsylvania ............ & 1,200 & 0 & 19.8 & 6.75 & 59.7 & 792 & 0 & 10.5 & 0 & 5,390 & 0 & 7,480 & 0 & 7,480 \\
\hline Rhode Island .............. & 92.2 & 0 & 0.39 & 0.01 & 8.90 & 3.35 & 0 & 0.49 & 0 & 1.44 & 232 & 98.0 & 241 & 339 \\
\hline South Carolina ......... & 504 & 0 & 57.4 & 6.79 & 8.97 & 365 & 0 & 1.74 & 0 & 5,500 & 0 & 6,440 & 0 & 6,440 \\
\hline South Dakota............ & 49.9 & 0 & 165 & 28.3 & 23.6 & 2.63 & 0 & 11.0 & 0 & 6.93 & 0 & 287 & 0 & 287 \\
\hline Tennessee .................... & 618 & 0 & 27.6 & 13.4 & 37.2 & 728 & 0 & 7.73 & 0 & 5,800 & 0 & 7,230 & 0 & 7,230 \\
\hline 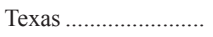 & 2,860 & 0 & 1,730 & 127 & 22.2 & 571 & 608 & 81.2 & 0.49 & 10,400 & 661 & 15,800 & 1,280 & 17,100 \\
\hline 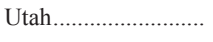 & 309 & 0 & 2,730 & 8.76 & 0 & 16.4 & 33.1 & 1.60 & 205 & 45.6 & 0.47 & 3,110 & 238 & 3,340 \\
\hline Vermont........................ & 29.2 & 0 & 1.68 & 1.41 & 4.96 & 3.69 & 0 & 3.53 & 0 & 344 & 0 & 389 & 0 & 389 \\
\hline 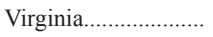 & 594 & 0 & 45.4 & 20.8 & 286 & 309 & 56.1 & 28.4 & 0 & 2,850 & 3,150 & 4,140 & 3,200 & 7,340 \\
\hline Washington................. & 439 & 0.02 & 2,350 & 8.55 & 127 & 358 & 33.1 & 3.36 & 0 & 36.4 & 0 & 3,320 & 33.1 & 3,350 \\
\hline West Virginia............. & 155 & 0.63 & 0.04 & 3.42 & 40.6 & 729 & 0 & 9.00 & 0 & 2,470 & 0 & 3,410 & 0 & 3,410 \\
\hline Wisconsin .................... & 221 & 0 & 123 & 7.30 & 30.2 & 382 & 0 & 8.63 & 0 & 4,630 & 0 & 5,400 & 0 & 5,400 \\
\hline 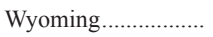 & 47.5 & 0 & 3,930 & 10.3 & 18.7 & 1.82 & 0 & 13.0 & 0 & 61.1 & 0 & 4,080 & 0 & 4,080 \\
\hline Puerto Rico................ & 590 & 0 & 15.7 & 2.24 & 0.40 & 0 & 0 & 0.18 & 0 & 2.61 & 2,270 & 611 & 2,270 & 2,880 \\
\hline U.S. Virgin Islands.. & 4.95 & 2.67 & 0 & 0.01 & 0 & 0 & 2.62 & 0 & 0.04 & 0.17 & 116 & 2.85 & 124 & 127 \\
\hline TOTAL & 26,300 & 63.9 & 65,900 & 797 & 7,610 & 12,100 & 937 & 1,130 & 282 & 116,000 & 43,800 & 230,000 & 45,000 & 275,000 \\
\hline
\end{tabular}


Table 3B. Surface-water withdrawals by water-use category, 2010, in thousand acre-feet per year.

[Values may not sum to totals because of independent rounding]

\begin{tabular}{|c|c|c|c|c|c|c|c|c|c|c|c|c|c|c|}
\hline \multirow[t]{2}{*}{ State } & \multirow{2}{*}{$\begin{array}{l}\text { Public } \\
\text { supply }\end{array}$} & \multirow{2}{*}{$\begin{array}{c}\text { Self- } \\
\text { supplied } \\
\text { domestic }\end{array}$} & \multirow{2}{*}{ Irrigation } & \multirow{2}{*}{$\begin{array}{l}\text { Live- } \\
\text { stock }\end{array}$} & \multirow{2}{*}{$\begin{array}{l}\text { Aqua- } \\
\text { culture }\end{array}$} & \multicolumn{2}{|c|}{$\begin{array}{l}\text { Self-supplied } \\
\text { industrial }\end{array}$} & \multicolumn{2}{|c|}{ Mining } & \multicolumn{2}{|c|}{$\begin{array}{c}\text { Thermoelectric } \\
\text { power }\end{array}$} & \multicolumn{3}{|c|}{ Total } \\
\hline & & & & & & Fresh & Saline & Fresh & Saline & Fresh & Saline & Fresh & Saline & Total \\
\hline Alabama ......................... & 617 & 0 & 83.0 & 16.6 & 29.9 & 606 & 0 & 8.40 & 0 & 9,250 & 0 & 10,600 & 0 & 10,600 \\
\hline Alaska ............................. & 58.1 & 0.74 & 0.02 & 0.17 & 285 & 4.93 & 4.82 & 27.0 & 85.6 & 62.6 & 0 & 439 & 90.4 & 529 \\
\hline Arizona.......................... & 704 & 0 & 3,220 & 0 & 8.71 & 0 & 0 & 0 & 0 & 30.4 & 0 & 3,970 & 0 & 3,970 \\
\hline Arkansas........................ & 331 & 0 & 1,500 & 26.2 & 96.9 & 240 & 0 & 49.4 & 0 & 1,720 & 0 & 3,970 & 0 & 3,970 \\
\hline 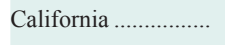 & 3,890 & 33.0 & 16,100 & 116 & 899 & 1.27 & 0 & 13.7 & 0.06 & 36.1 & 7,270 & 21,100 & 7,270 & 28,400 \\
\hline Colorado......................... & 804 & 0 & 9,440 & 13.2 & 111 & 142 & 0 & 3.42 & 0 & 67.5 & 0 & 10,600 & 0 & 10,600 \\
\hline Connecticut ................. & 327 & 0 & 25.9 & 0 & 25.8 & 67.5 & 43.1 & 4.26 & 0 & 222 & 2,750 & 673 & 2,800 & 3,470 \\
\hline Delaware ..................... & 37.3 & 0 & 17.1 & 0 & 0 & 98.1 & 0 & 0.46 & 0 & 8.35 & 468 & 161 & 468 & 629 \\
\hline District of Columbia & 0 & 0 & 0.06 & 0 & 0 & 0 & 0 & 0 & 0 & 0 & 0 & 0.06 & 0 & 0.06 \\
\hline Florida ............................. & 287 & 0 & 1,500 & 2.49 & 0 & 53.5 & 0 & 38.2 & 0 & 639 & 9,600 & 2,500 & 9,620 & 12,100 \\
\hline Georgia ............................ & 979 & 0 & 227 & 30.1 & 51.4 & 315 & 0 & 9.43 & 0 & 1,980 & 317 & 3,600 & 317 & 3,910 \\
\hline 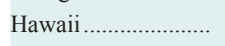 & 17.7 & 6.92 & 249 & 1.35 & 2.69 & 0 & 0 & 0.12 & 0 & 0 & 619 & 278 & 619 & 897 \\
\hline 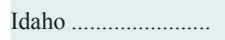 & 30.4 & 0 & 11,500 & 10.1 & 3,010 & 19.2 & 0 & 21.2 & 0 & 0 & 0 & 14,600 & 0 & 14,600 \\
\hline Illinois ........................... & 1,280 & 0 & 19.6 & 0.03 & 30.5 & 299 & 0 & 62.1 & 0 & 12,000 & 0 & 13,700 & 0 & 13,700 \\
\hline Indiana........................... & 341 & 0 & 43.4 & 14.6 & 2.21 & 2,380 & 0 & 93.9 & 0 & 6,010 & 0 & 8,880 & 0 & 8,880 \\
\hline 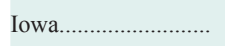 & 94.5 & 0 & 1.32 & 37.9 & 4.99 & 3.03 & 0 & 87.5 & 0 & 2,480 & 0 & 2,710 & 0 & 2,710 \\
\hline 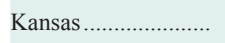 & 259 & 0 & 179 & 25.8 & 9.61 & 7.61 & 0 & 4.46 & 0 & 410 & 0 & 897 & 0 & 897 \\
\hline Kentucky .................... & 562 & 15.1 & 30.7 & 46.6 & 37.6 & 164 & 0 & 25.7 & 0 & 3,740 & 0 & 4,630 & 0 & 4,630 \\
\hline 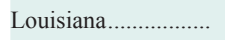 & 413 & 0 & 289 & 4.35 & 127 & 2,050 & 0 & 6.66 & 0 & 4,920 & 1.88 & 7,810 & 1.88 & 7,810 \\
\hline Maine ….......................... & 71.3 & 0 & 9.83 & 0.65 & 23.7 & 208 & 16.6 & 4.18 & 0 & 29.0 & 29.1 & 347 & 45.8 & 392 \\
\hline Maryland ....................... & 785 & 0 & 20.9 & 2.50 & 17.6 & 43.3 & 164 & 2.44 & 0 & 486 & 6,460 & 1,360 & 6,630 & 7,980 \\
\hline Massachusetts .......... & 548 & 0 & 24.0 & 0.56 & 47.6 & 13.5 & 0 & 5.36 & 0 & 150 & 2,160 & 788 & 2,170 & 2,950 \\
\hline Michigan ..................... & 990 & 0 & 70.1 & 2.13 & 88.0 & 602 & 0 & 74.0 & 0 & 9,540 & 0 & 11,400 & 0 & 11,400 \\
\hline 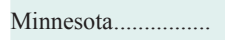 & 211 & 0 & 29.9 & 0 & 17.0 & 80.4 & 0 & 310 & 0 & 2,810 & 0 & 3,460 & 0 & 3,460 \\
\hline Mississippi ................... & 51.9 & 0 & 149 & 12.4 & 21.6 & 140 & 0 & 0.62 & 0 & 1,020 & 70.0 & 1,390 & 70.0 & 1,460 \\
\hline Missouri ........................... & 609 & 0 & 55.6 & 61.0 & 191 & 38.3 & 0 & 9.43 & 0 & 6,610 & 0 & 7,570 & 0 & 7,570 \\
\hline Montana ....................... & 81.1 & 1.17 & 7,880 & 33.0 & 18.4 & 33.1 & 0 & 29.3 & 0 & 168 & 0 & 8,250 & 0 & 8,250 \\
\hline 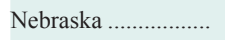 & 68.8 & 0 & 1,520 & 23.7 & 92.1 & 2.61 & 0 & 9.83 & 0 & 2,010 & 0 & 3,730 & 0 & 3,730 \\
\hline 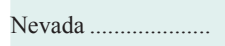 & 502 & 0 & 1,030 & 0 & 43.5 & 5.08 & 0 & 4.61 & 0 & 4.13 & 0 & 1,590 & 0 & 1,590 \\
\hline New Hampshire ....... & 63.4 & 0 & 0.75 & 0.25 & 9.51 & 7.91 & 0 & 3.18 & 0 & 225 & 951 & 310 & 951 & 1,260 \\
\hline New Jersey ................... & 765 & 0 & 78.5 & 0 & 0 & 54.4 & 0 & 7.75 & 0 & 574 & 4,190 & 1,480 & 4,190 & 5,670 \\
\hline New Mexico............... & 81.1 & 0 & 1,640 & 3.40 & 4.84 & 0.93 & 0 & 10.9 & 0 & 47.4 & 0 & 1,780 & 0 & 1,780 \\
\hline 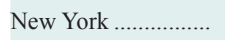 & 2,020 & 0 & 45.0 & 8.97 & 41.3 & 354 & 0 & 71.8 & 0 & 3,090 & 5,430 & 5,630 & 5,430 & 11,100 \\
\hline North Carolina ......... & 858 & 0 & 312 & 16.8 & 1,630 & 210 & 0 & 5.46 & 0 & 8,580 & 1,530 & 11,600 & 1,530 & 13,100 \\
\hline North Dakota............ & 42.9 & 0 & 97.8 & 9.66 & 6.64 & 14.5 & 0 & 5.19 & 0 & 938 & 0 & 1,110 & 0 & 1,110 \\
\hline Ohio...................................... & 1,030 & 3.08 & 39.7 & 18.2 & 21.3 & 328 & 0 & 40.2 & 0 & 8,060 & 0 & 9,540 & 0 & 9,540 \\
\hline 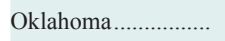 & 591 & 0 & 151 & 63.1 & 8.33 & 16.0 & 0 & 14.9 & 0 & 431 & 0 & 1,270 & 0 & 1,270 \\
\hline Oregon................................... & 471 & 7.93 & 3,750 & 15.7 & 761 & 138 & 0 & 1.31 & 0 & 12.6 & 0 & 5,160 & 0 & 5,160 \\
\hline Pennsylvania .............. & 1,340 & 0 & 22.1 & 7.57 & 66.9 & 888 & 0 & 11.8 & 0 & 6,040 & 0 & 8,380 & 0 & 8,380 \\
\hline Rhode Island ............... & 103 & 0 & 0.44 & 0.01 & 9.98 & 3.76 & 0 & 0.55 & 0 & 1.61 & 260 & 110 & 270 & 380 \\
\hline South Carolina .......... & 565 & 0 & 64.4 & 7.61 & 10.1 & 409 & 0 & 1.95 & 0 & 6,160 & 0 & 7,220 & 0 & 7,220 \\
\hline South Dakota............. & 56.0 & 0 & 185 & 31.7 & 26.4 & 2.95 & 0 & 12.3 & 0 & 7.77 & 0 & 322 & 0 & 322 \\
\hline Tennessee ................... & 692 & 0 & 31.0 & 15.1 & 41.7 & 817 & 0 & 8.67 & 0 & 6,500 & 0 & 8,100 & 0 & 8,100 \\
\hline 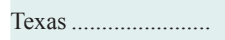 & 3,200 & 0 & 1,940 & 143 & 24.9 & 641 & 682 & 91.0 & 0.55 & 11,700 & 741 & 17,700 & 1,430 & 19,200 \\
\hline 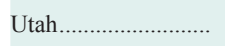 & 346 & 0 & 3,060 & 9.82 & 0 & 18.4 & 37.1 & 1.79 & 229 & 51.1 & 0.53 & 3,480 & 267 & 3,750 \\
\hline Vermont......................... & 32.7 & 0 & 1.88 & 1.58 & 5.56 & 4.14 & 0 & 3.96 & 0 & 386 & 0 & 436 & 0 & 436 \\
\hline 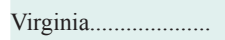 & 666 & 0 & 50.8 & 23.4 & 320 & 346 & 62.9 & 31.8 & 0 & 3,200 & 3,530 & 4,640 & 3,590 & 8,230 \\
\hline 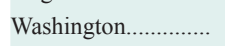 & 492 & 0.02 & 2,630 & 9.58 & 142 & 402 & 37.1 & 3.77 & 0 & 40.8 & 0 & 3,720 & 37.1 & 3,760 \\
\hline West Virginia.............. & 174 & 0.71 & 0.04 & 3.83 & 45.5 & 817 & 0 & 10.1 & 0 & 2,770 & 0 & 3,820 & 0 & 3,820 \\
\hline Wisconsin ...................... & 247 & 0 & 138 & 8.18 & 33.9 & 428 & 0 & 9.67 & 0 & 5,190 & 0 & 6,060 & 0 & 6,060 \\
\hline 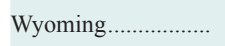 & 53.3 & 0 & 4,410 & 11.6 & 21.0 & 2.04 & 0 & 14.6 & 0 & 68.4 & 0 & 4,580 & 0 & 4,580 \\
\hline Puerto Rico................... & 661 & 0 & 17.6 & 2.51 & 0.45 & 0 & 0 & 0.20 & 0 & 2.93 & 2,540 & 685 & 2,540 & 3,230 \\
\hline U.S. Virgin Islands.. & 5.55 & 2.99 & 0 & 0.01 & 0 & 0 & 2.94 & 0 & 0.04 & 0.19 & 130 & 3.19 & 139 & 142 \\
\hline TOTAL & 29,500 & 71.7 & 73,900 & 893 & 8,530 & 13,500 & 1,050 & 1,270 & 316 & 130,000 & 49,100 & 258,000 & 50,500 & 309,000 \\
\hline
\end{tabular}


Table 4A. Groundwater withdrawals by water-use category, 2010, in million gallons per day.

[Values may not sum to totals because of independent rounding]

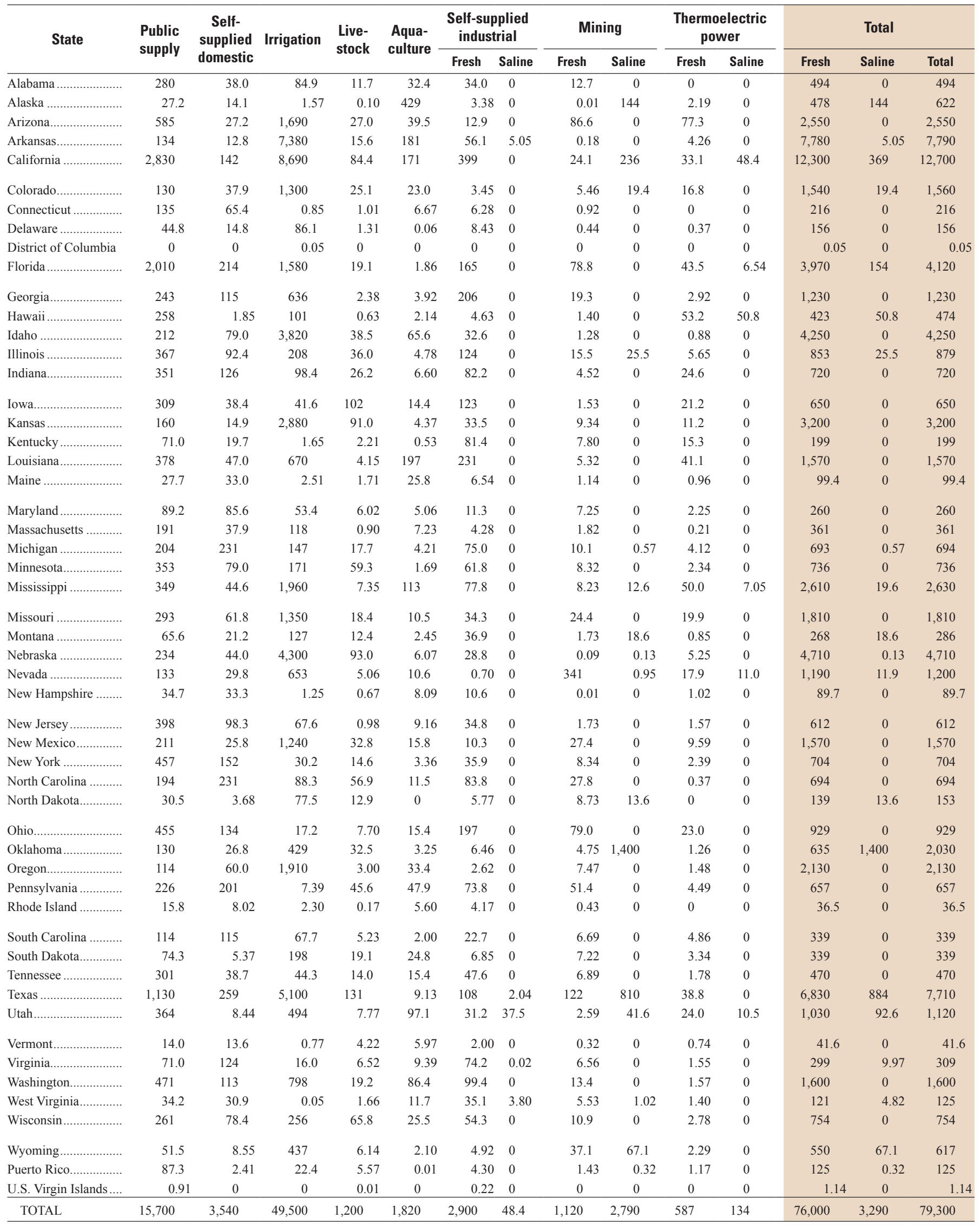


Table 4B. Groundwater withdrawals by water-use category, 2010, in thousand acre-feet per year.

[Values may not sum to totals because of independent rounding]

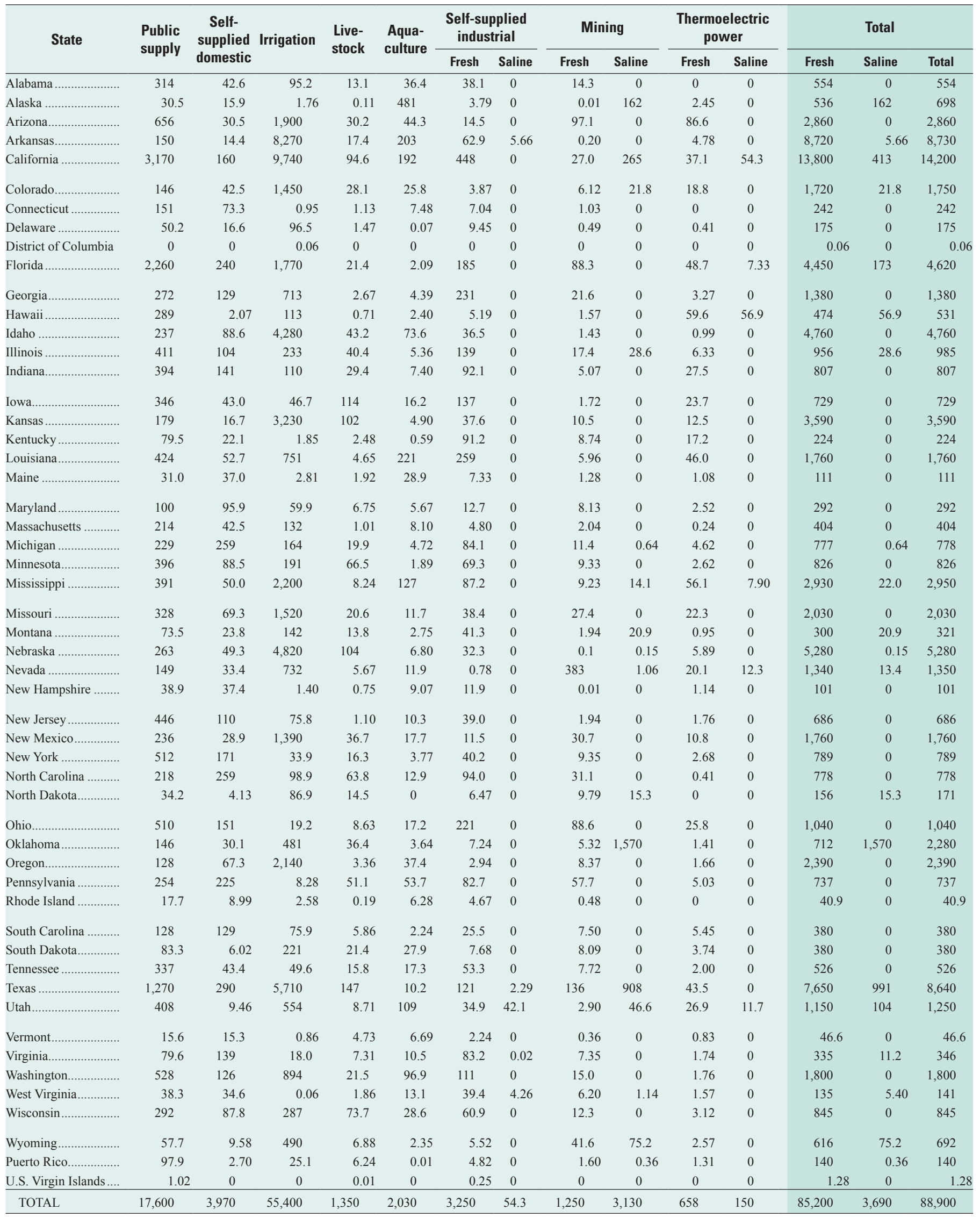



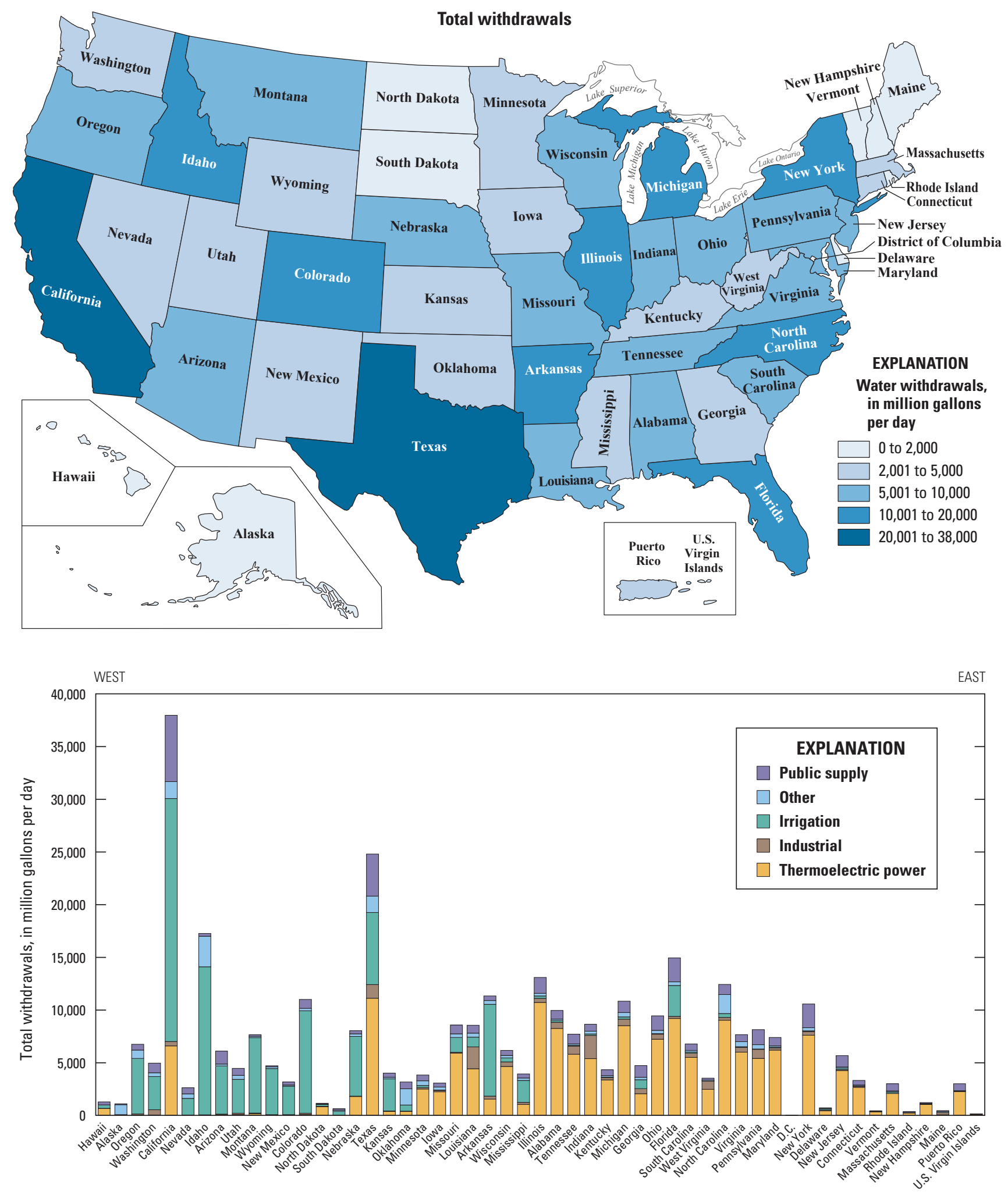

Figure 2. Total water withdrawals by State and barchart showing categories by State from west to east, 2010 . 

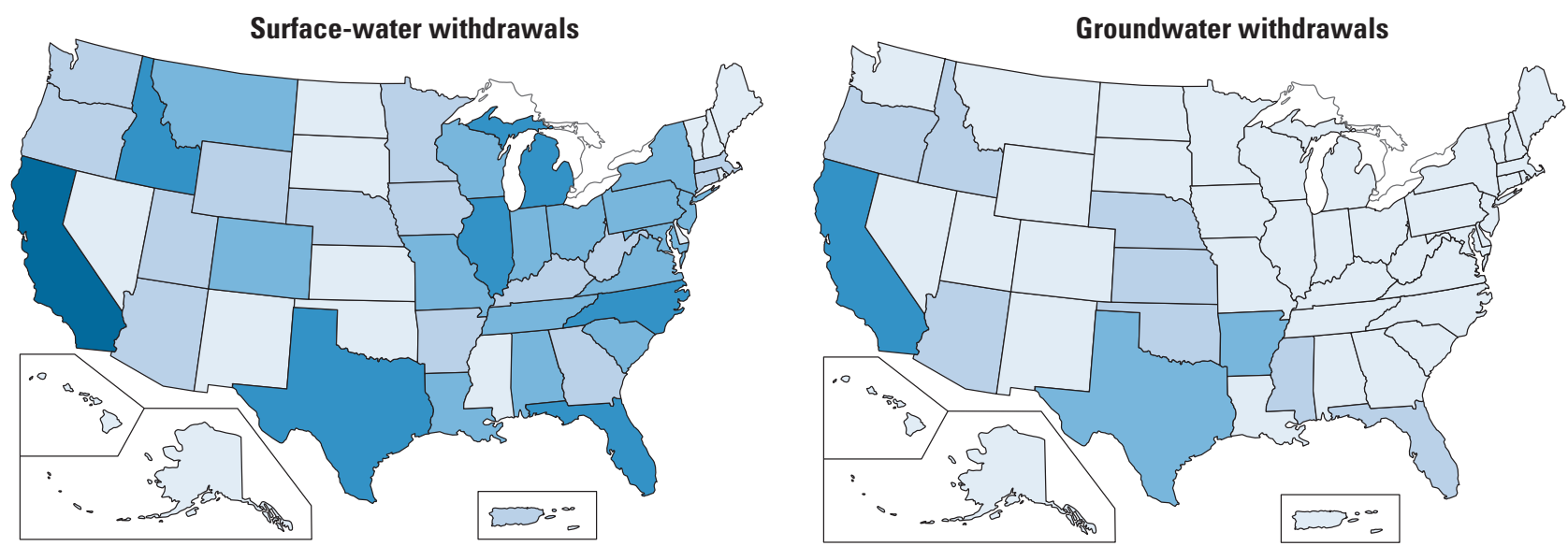

EXPLANATION

Water withdrawals, in million gallons per day

\begin{tabular}{|l}
\hline \\
0 to 2,000 \\
2,001 to 5,000 \\
5,001 to 10,000 \\
10,001 to 20,000 \\
20,001 to 38,000
\end{tabular}
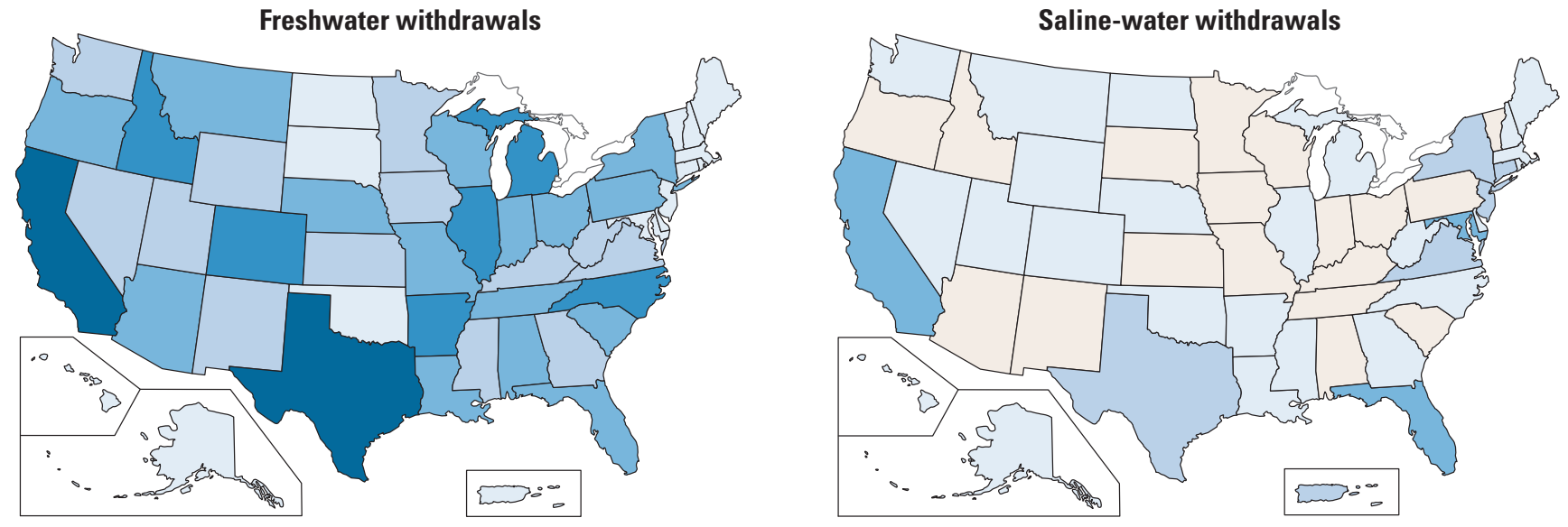

EXPLANATION

Water withdrawals, in million gallons per day

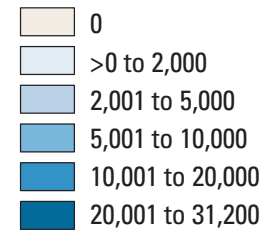

Figure 3. Surface-water and groundwater, and freshwater and saline-water withdrawals, 2010. 
Public supply refers to water withdrawn by public and private water suppliers that provide water to at least 25 people or have a minimum of 15 connections. Public-supply water is delivered to users for domestic, commercial, and industrial purposes, and also is used for public services and system losses.

Approximately $42,000 \mathrm{Mgal} / \mathrm{d}$ (table 5), or 47,100 thousand acre-ft/yr (table $2 B$ ), of water were withdrawn for public supply in 2010. This amount is 5 percent less than the estimated amount of water withdrawn for public supply in 2005 . Public supply represents about 14 percent of total freshwater withdrawals and 22 percent of all withdrawals excluding thermoelectric power. In some States, public-supply water sources include desalinated seawater or brackish groundwater that has been treated to reduce dissolved solids. A combined total of 23.5 Mgal/d saline surface-water withdrawals for public-supply use were reported in Florida, the U.S. Virgin Islands, Massachusetts, and Texas. A combined total of $317 \mathrm{Mgal} / \mathrm{d}$ saline groundwater withdrawals for public-supply use were identified in Florida, California, Texas, Virginia, and Utah. Because these saline withdrawals were identified for only seven States and represent less than 1 percent of total public-supply withdrawals, they are not listed separately in table 5 but were included in the calculations.

An estimated 268 million people relied on publicsupply water for their household use in 2010. This number represents about 86 percent of the total U.S. population. About 35 percent of all public-supply withdrawals were in the four States with the largest populations: California, Texas, New York, and Florida (fig. 4). Sixty-three percent of water withdrawn for public supply in 2010 was from surface sources, such as lakes and streams; the other 37 percent was from groundwater.

Five States-California, Texas, New York, Pennsylvania, and Illinois - each withdrew more than $1,000 \mathrm{Mgal} / \mathrm{d}$ of surface water for public supply in 2010 and together accounted for 40 percent of the total surface-water withdrawals for public supply. In 36 States, including Puerto Rico and the U.S. Virgin Islands, surface-water sources provided more than half of the total public-supply withdrawals.

Three States - California, Florida, and Texas - each withdrew more than $1,000 \mathrm{Mgal} / \mathrm{d}$ of groundwater for public supply in 2010 and accounted for 38 percent of total groundwater withdrawals for public supply. States that relied on groundwater for 75 percent or more of their public-supply withdrawals were Hawaii, Florida, Idaho, Mississippi, Nebraska, and Iowa.

Most of the public-supply withdrawals are delivered to customers for domestic, commercial, and industrial needs. Part of the total is used for public services, such as public pools, parks, firefighting, water and wastewater treatment, and municipal buildings, and some is unaccounted for because of leaks, flushing, tower maintenance, and other system losses. Domestic deliveries represent the largest single component of public-supply withdrawals, averaging 57 percent of the total nationally. Estimates of publicsupply deliveries to domestic use, representing indoor and outdoor water uses at occupied residences, are identified in table 5. Estimates for commercial and industrial deliveries, public use, and system losses were not available for all States and, therefore, are included in table 5 as an aggregate number.

Methods for estimating public-supply withdrawals, source of water, population served, and domestic deliveries varied by State. Common sources of information about withdrawals by source included data collected from water suppliers by State water regulatory agencies or through surveys. Estimates of the population served by public supply were derived using various sources, including reports from State agencies, the EPA SDWIS database, U.S. Census data, and information on service connections from public suppliers. Methods for estimating domestic deliveries included surveys of publicsupply sales information, calculations using coefficients for per capita use, and development of average percentages of deliveries to various customer categories. 
Table 5. Public-supply water withdrawals, 2010.

[Values may not sum to totals because of independent rounding; Mgal/d, million gallons per day; n/a, not applicable]

\begin{tabular}{|c|c|c|c|c|c|c|c|c|c|}
\hline \multirow{3}{*}{ State } & \multicolumn{3}{|c|}{ Population (in thousands) } & \multicolumn{3}{|c|}{ Withdrawals (in Mgal/d) } & \multicolumn{3}{|c|}{ Public-supply deliveries } \\
\hline & \multirow[b]{2}{*}{ Total } & \multicolumn{2}{|c|}{ Served by public supply } & \multicolumn{2}{|c|}{ By source } & \multirow[b]{2}{*}{ Total } & \multirow{2}{*}{$\begin{array}{l}\text { Domestic use } \\
\text { (in Mgal/d) }\end{array}$} & \multirow{2}{*}{$\begin{array}{c}\text { Domestic use } \\
\text { (in percent) }\end{array}$} & \multirow{2}{*}{$\begin{array}{c}\text { All other uses } \\
\text { and system } \\
\text { losses (in Mgal/d) }\end{array}$} \\
\hline & & Population & $\begin{array}{l}\text { Population } \\
\text { (in percent) }\end{array}$ & $\begin{array}{l}\text { Ground- } \\
\text { water }\end{array}$ & $\begin{array}{c}\text { Surface } \\
\text { water }\end{array}$ & & & & \\
\hline Alabama .............................. & 4,780 & 4,240 & 89 & 280 & 551 & 831 & 327 & 39 & 504 \\
\hline Alaska .............................. & 710 & 450 & 63 & 27.2 & 51.8 & 79.0 & 49.2 & 62 & 29.9 \\
\hline 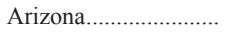 & 6,390 & 6,170 & 97 & 585 & 628 & 1,210 & 912 & 75 & 301 \\
\hline Arkansas........................... & 2,920 & 2,770 & 95 & 134 & 295 & 429 & 295 & 69 & 133 \\
\hline California ...................... & 37,300 & 34,800 & 93 & 2,830 & 3,470 & 6,300 & 3,870 & 61 & 2,430 \\
\hline Colorado........................ & 5,030 & 4,720 & 94 & 130 & 717 & 848 & 521 & 61 & 327 \\
\hline Connecticut ................ & 3,570 & 2,700 & 76 & 135 & 292 & 427 & 203 & 47 & 224 \\
\hline Delaware ......................... & 898 & 713 & 79 & 44.8 & 33.3 & 78.1 & 57.0 & 73 & 21.0 \\
\hline District of Columbia & 602 & 602 & 100 & 0 & 0 & 0 & 74.9 & $\mathrm{n} / \mathrm{a}$ & $\mathrm{n} / \mathrm{a}$ \\
\hline 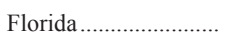 & 18,800 & 16,900 & 90 & 2,010 & 256 & 2,270 & 1,430 & 63 & 838 \\
\hline 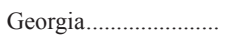 & 9,690 & 8,160 & 84 & 243 & 873 & 1,120 & 651 & 58 & 465 \\
\hline 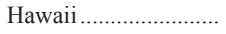 & 1,360 & 1,300 & 96 & 258 & 15.8 & 274 & 188 & 69 & 86.0 \\
\hline Idaho ................................ & 1,570 & 1,140 & 72 & 212 & 27.1 & 239 & 184 & 77 & 54.2 \\
\hline Illinois .......................... & 12,800 & 11,700 & 91 & 367 & 1,140 & 1,500 & 934 & 62 & 571 \\
\hline Indiana........................... & 6,480 & 4,830 & 74 & 351 & 304 & 656 & 367 & 56 & 289 \\
\hline 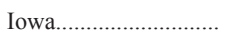 & 3,050 & 2,450 & 81 & 309 & 84.3 & 393 & 160 & 41 & 233 \\
\hline 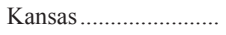 & 2,850 & 2,700 & 95 & 160 & 231 & 391 & 194 & 50 & 197 \\
\hline Kentucky .......................... & 4,340 & 3,680 & 85 & 71.0 & 501 & 572 & 257 & 45 & 315 \\
\hline Louisiana....................... & 4,530 & 3,950 & 87 & 378 & 368 & 746 & 426 & 57 & 321 \\
\hline Maine ….......................... & 1,330 & 768 & 58 & 27.7 & 63.6 & 91.3 & 39.4 & 43 & 51.9 \\
\hline 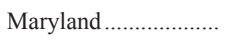 & 5,770 & 4,700 & 81 & 89.2 & 701 & 790 & 506 & 64 & 283 \\
\hline Massachusetts ............. & 6,550 & 6,010 & 92 & 191 & 489 & 679 & 385 & 57 & 294 \\
\hline Michigan ............................ & 9,880 & 7,210 & 73 & 204 & 883 & 1,090 & 548 & 50 & 540 \\
\hline Minnesota..................... & 5,300 & 4,180 & 79 & 353 & 188 & 542 & 248 & 46 & 294 \\
\hline 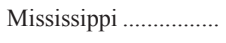 & 2,970 & 2,520 & 85 & 349 & 46.3 & 395 & 252 & 64 & 143 \\
\hline 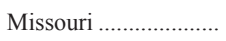 & 5,990 & 5,110 & 85 & 293 & 543 & 836 & 467 & 56 & 369 \\
\hline Montana ....................... & 989 & 704 & 71 & 65.6 & 72.4 & 138 & 83.2 & 60 & 54.7 \\
\hline Nebraska ....................... & 1,830 & 1,480 & 81 & 234 & 61.4 & 296 & 130 & 44 & 165 \\
\hline 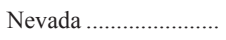 & 2,700 & 2,540 & 94 & 133 & 448 & 581 & 331 & 57 & 250 \\
\hline New Hampshire .......... & 1,320 & 871 & 66 & 34.7 & 56.6 & 91.2 & 58.6 & 64 & 32.6 \\
\hline 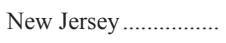 & 8,790 & 7,830 & 89 & 398 & 682 & 1,080 & 605 & 56 & 475 \\
\hline New Mexico................. & 2,060 & 1,760 & 85 & 211 & 72.4 & 283 & 160 & 57 & 123 \\
\hline New York ...................... & 19,400 & 17,300 & 89 & 457 & 1,810 & 2,260 & 1,370 & 61 & 889 \\
\hline North Carolina ............ & 9,540 & 6,230 & 65 & 194 & 766 & 960 & 436 & 45 & 524 \\
\hline North Dakota................... & 673 & 623 & 93 & 30.5 & 38.3 & 68.8 & 49.9 & 72 & 19.0 \\
\hline Ohio.............................. & 11,500 & 9,710 & 84 & 455 & 918 & 1,370 & 619 & 45 & 755 \\
\hline Oklahoma ...................... & 3,750 & 3,440 & 92 & 130 & 527 & 657 & 292 & 44 & 365 \\
\hline Oregon.......................... & 3,830 & 3,220 & 84 & 114 & 420 & 534 & 365 & 68 & 169 \\
\hline Pennsylvania ................. & 12,700 & 9,360 & 74 & 226 & 1,200 & 1,420 & 548 & 38 & 877 \\
\hline Rhode Island .................. & 1,050 & 940 & 89 & 15.8 & 92.2 & 108 & 67.6 & 63 & 40.4 \\
\hline South Carolina ............. & 4,630 & 3,470 & 75 & 114 & 504 & 619 & 347 & 56 & 271 \\
\hline South Dakota................. & 814 & 739 & 91 & 74.3 & 49.9 & 124 & 70.7 & 57 & 53.5 \\
\hline Tennessee ..................... & 6,350 & 5,810 & 92 & 301 & 618 & 918 & 470 & 51 & 448 \\
\hline Texas ................................... & 25,100 & 22,700 & 90 & 1,130 & 2,860 & 3,990 & 2,050 & 51 & 1,940 \\
\hline Utah & 2,760 & 2,710 & 98 & 364 & 309 & 673 & 453 & 67 & 220 \\
\hline 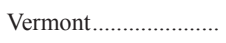 & 626 & 444 & 71 & 14.0 & 29.2 & 43.1 & 26.3 & 61 & 16.8 \\
\hline 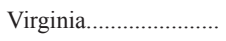 & 8,000 & 6,350 & 79 & 71.0 & 594 & 665 & 476 & 72 & 188 \\
\hline Washington.................... & 6,720 & 5,720 & 85 & 471 & 439 & 910 & 634 & 70 & 276 \\
\hline West Virginia.................. & 1,850 & 1,460 & 79 & 34.2 & 155 & 189 & 117 & 62 & 72.4 \\
\hline Wisconsin ........................ & 5,690 & 4,040 & 71 & 261 & 221 & 481 & 211 & 44 & 270 \\
\hline Wyoming ............................ & 564 & 450 & 80 & 51.5 & 47.5 & 99.0 & 72.9 & 74 & 26.1 \\
\hline Puerto Rico................... & 3,730 & 3,690 & 99 & 87.3 & 590 & 677 & 230 & 34 & 447 \\
\hline U.S. Virgin Islands .... & 106 & 69.0 & 65 & 0.91 & 4.95 & 5.86 & 3.67 & 63 & 2.19 \\
\hline TOTAL & 313,000 & 268,000 & 86 & 15,700 & 26,300 & 42,000 & 23,800 & 57 & 18,200 \\
\hline
\end{tabular}



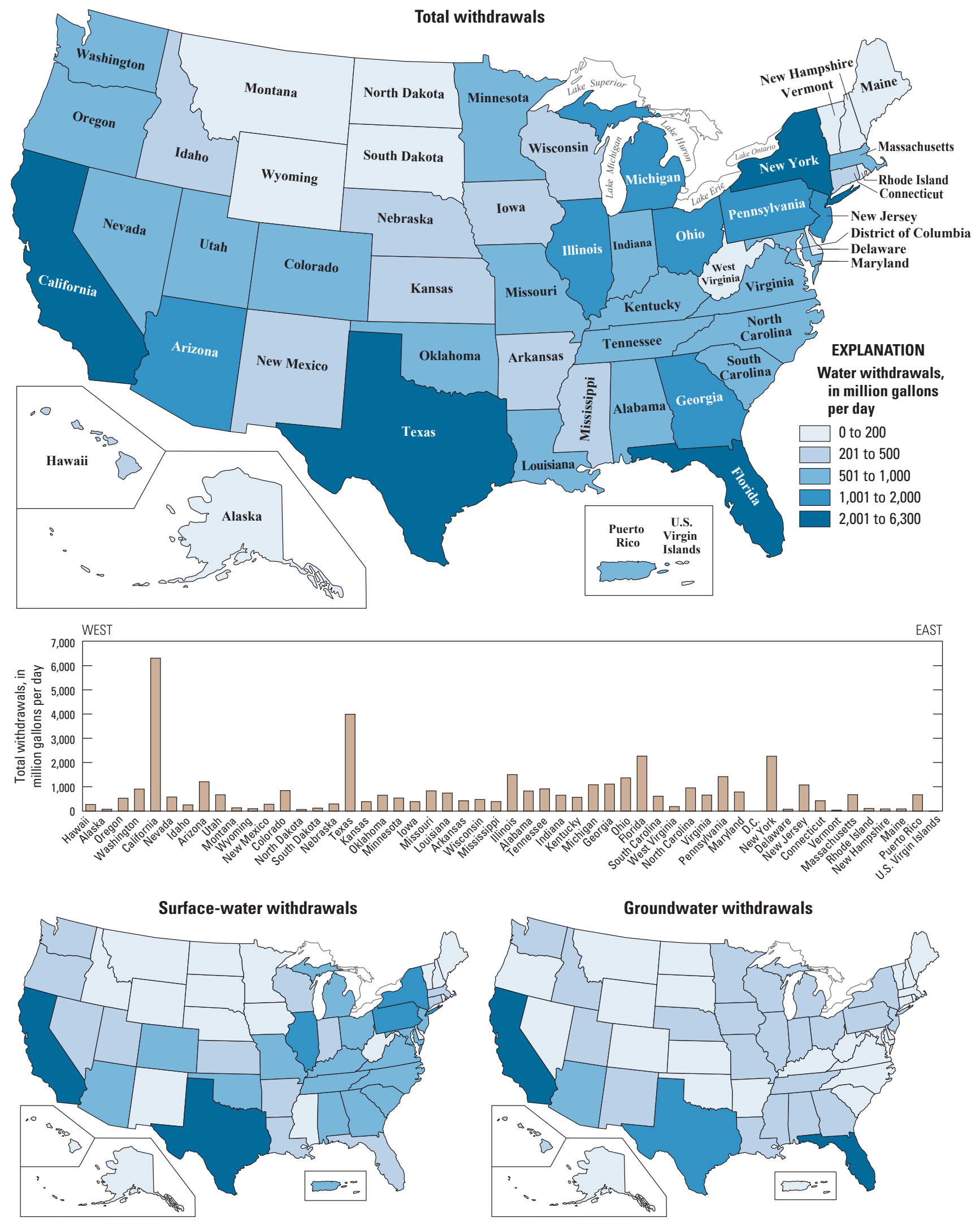

Figure 4. Public-supply withdrawals by source and State, 2010. 


\section{Domestic}

Domestic water use includes indoor and outdoor uses at residences. Common indoor water uses are drinking, food preparation, washing clothes and dishes, and flushing toilets. Common outdoor uses are watering lawns and gardens or maintaining pools, ponds, or other landscape features in a domestic environment. Domestic water is either self-supplied or provided by public suppliers. Self-supplied domestic water use is typically withdrawn from a private source, such as a well, or captured as rainwater in a cistern. Domestic deliveries are provided to homes by public suppliers. Figure 5 illustrates the proportions of total domestic water from public-supply deliveries and selfsupply domestic withdrawals.

Table 6 lists the estimated self-supplied and public-supplied population in each State, as well as the amounts used by each segment of the population for domestic needs and the respective per capita use in gallons per day (gallons per capita daily, gped). Domestic self-supplied withdrawals and public-supplied deliveries also are combined in table 6 to show the total estimated domestic use in 2010 and the weighted per capita use in gallons per day calculated for all domestic use.

An estimated 44.5 million people in the United States, or 14 percent of the population, provided their own water for domestic use in 2010. These self-supplied withdrawals were estimated at 3,600 Mgal/d (4,040 thousand acre-ft/yr), or about 1 percent of total withdrawals for all uses in 2010 . Nearly all (98 percent) of these self-supplied withdrawals were from fresh groundwater sources. Self-supplied domestic withdrawals are rarely metered or reported; typically this usage is calculated by multiplying an estimate of the population not served by public supply by a coefficient for daily per capita use. For some States, these coefficients were county-specific averages derived from observed residential water use and population estimates in nearby areas served by public suppliers. Other States used the same coefficient for all counties, commonly one used by State regulatory or planning agencies. Self-supplied domestic per capita use ranged from 48 gpcd in Wisconsin to 189 gpcd in Nevada. Generally, per capita use is least in the Northern and Eastern States and greatest in the Mountain and Western States where outdoor watering is more common. The national average selfsupplied domestic per capita use in 2010 was 81 gpcd (table 6).

The majority of people in the United States used water provided by public suppliers. Domestic deliveries by public water suppliers totaled 23,800 Mgal/d in 2010 and represented water provided to 268 million people at single-family and multifamily dwellings. Per capita water use for domestic deliveries ranged from 51 gpcd in Maine to 167 gpcd in Utah. The national average was 89 gpcd for public-supplied domestic water use. This per capita usage is less than the rate of $101 \mathrm{gpcd}$ observed in 1995 and 100 gpcd in 2005. Domestic deliveries from public supply were not compiled nationally in 2000.

Combined self-supplied domestic withdrawals and public-supply deliveries totaled 27,400 Mgal/d in 2010, and the national average per capita usage was 88 gpcd. The corresponding average per capita use for total domestic use in 2005 was 98 gpcd. The geographic distribution of total domestic water use by State is shown in figure $6 A$. Self-supplied domestic population in each State, in thousands of people and as a percentage of total State population, are shown in figure $6 B$. Self-supplied domestic populations were largest in Pennsylvania, North Carolina, and Michigan. States with the largest percentages of their population that were selfsupplied were Maine, Alaska, and the U.S. Virgin Islands.

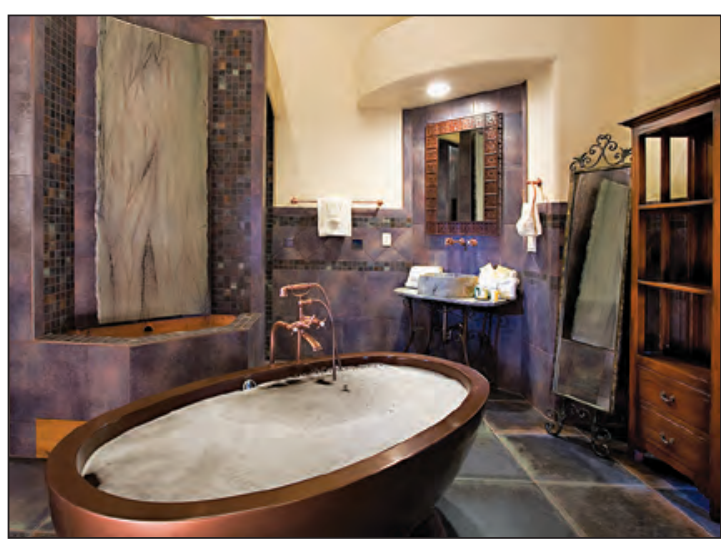

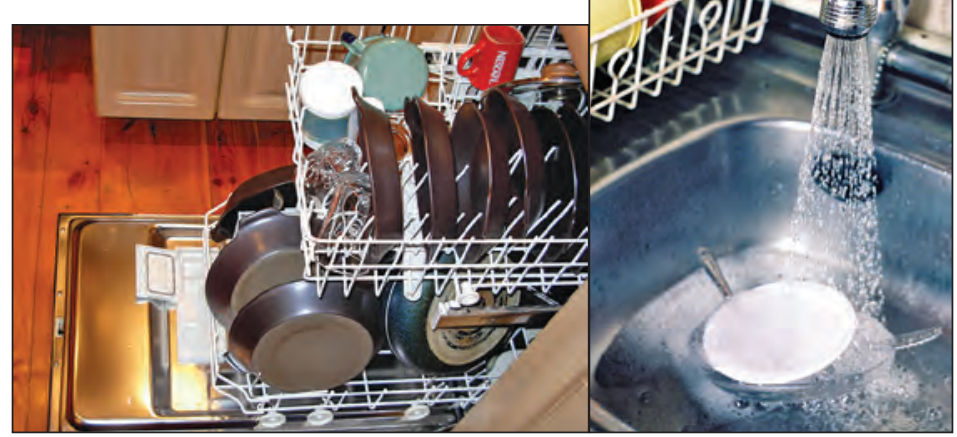

Domestic water use in the bathroom (left, photo used with permission from Tyrrell and Laning International, Inc.) and in the kitchen (right, photos from Wikimedia Commons). 
Table 6. Domestic water withdrawals and deliveries, 2010.

[Values may not sum to totals because of independent rounding; Mgal/d, million gallons per day; gal/d, gallons per day]

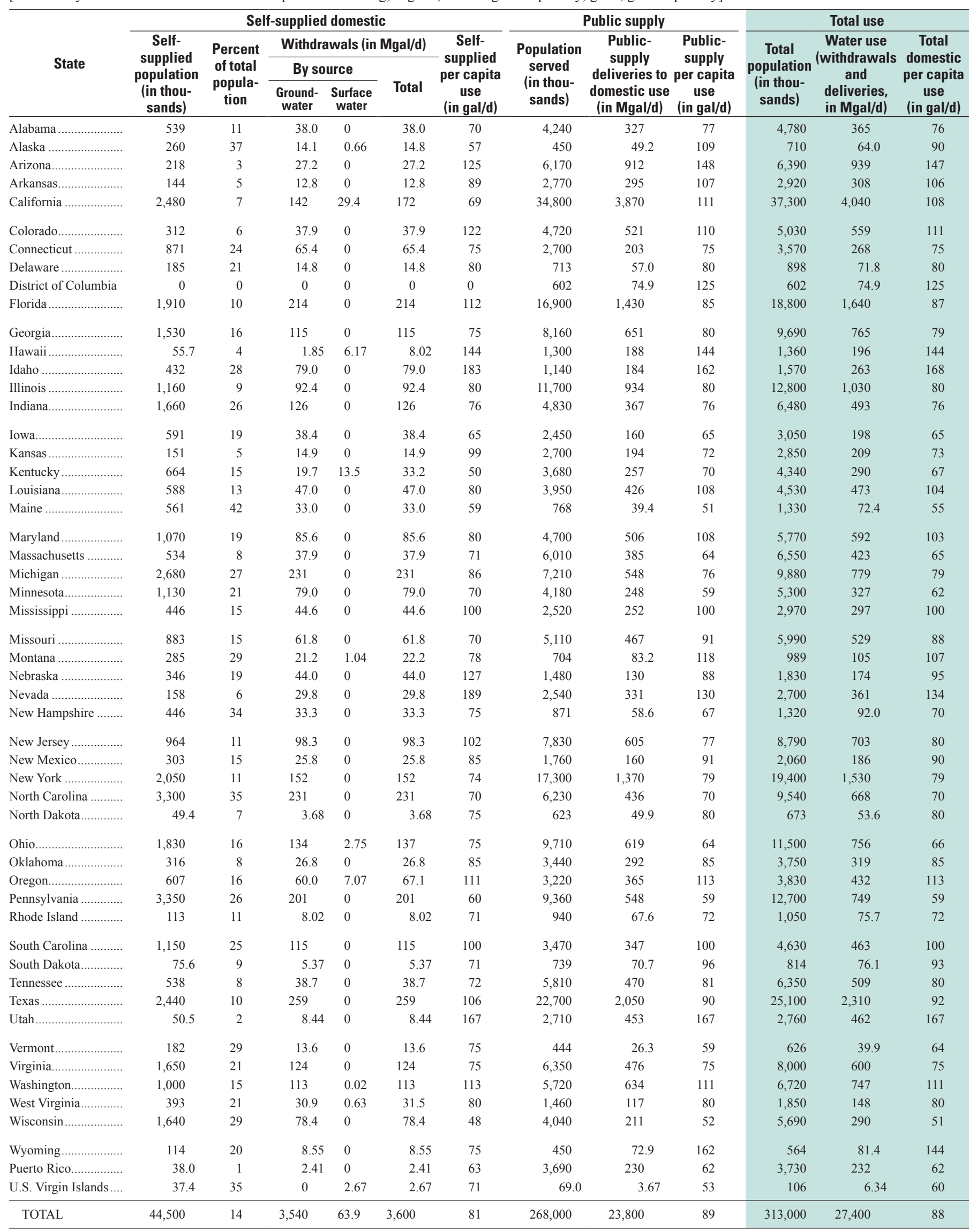



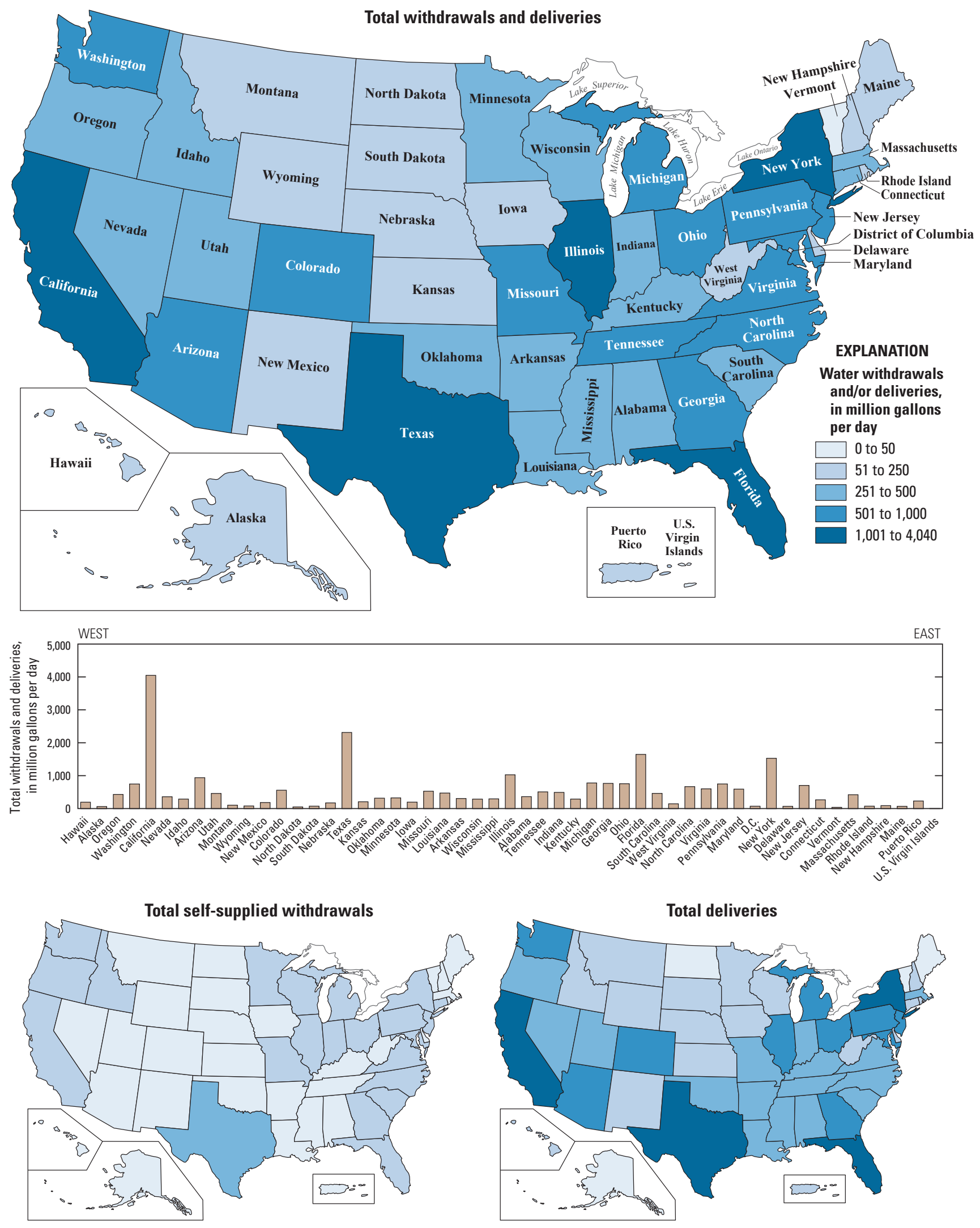

Figure 6A. Domestic withdrawals and deliveries by State, 2010. 

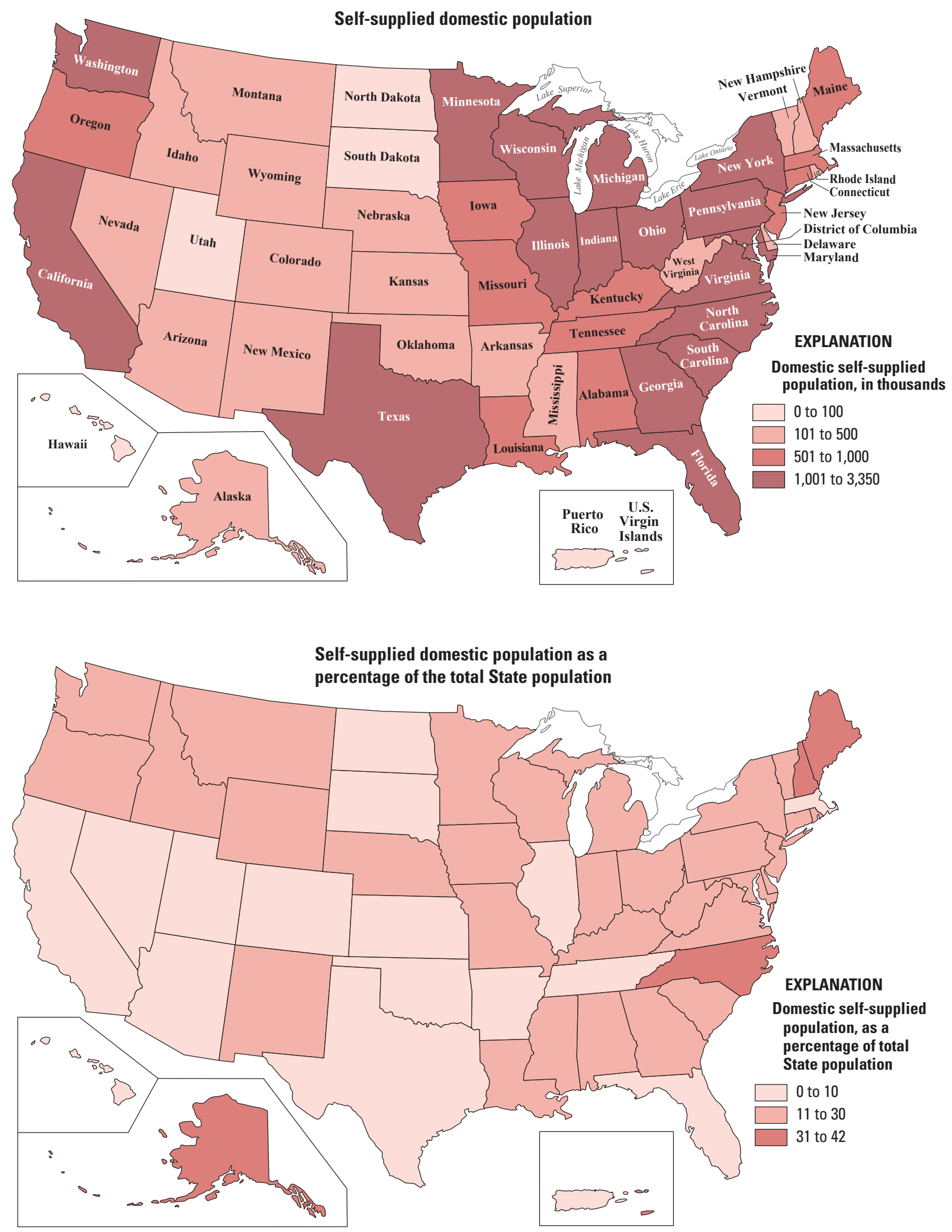

Figure 6B. Self-supplied domestic population and percentage of total population by State, 2010. 
Irrigation water use includes water that is applied by an irrigation system to sustain plant growth in all agricultural and horticultural practices. Irrigation also includes water that is used for pre-irrigation, frost protection, application of chemicals, weed control, field preparation, crop cooling, harvesting, dust suppression, and leaching salts from the root zone. Estimates of irrigation withdrawals include water that is lost in conveyance prior to application on fields as well as water that may subsequently return to a surface-water body as runoff after application, water consumed as evapotranspiration (ET) from plants and ground surfaces, or water that recharges aquifers as it seeps past the root zone. Irrigation of golf courses, parks, nurseries, turf farms, cemeteries, and other self-supplied landscape-watering uses also are included in the estimates. Irrigation water use includes self-supplied withdrawals and deliveries from irrigation companies or districts, cooperatives, or governmental entities. Some irrigation water is reclaimed wastewater from nearby treatment facilities or industries although these quantities are not included in irrigation withdrawals reported here. All irrigation withdrawals are considered freshwater. Irrigated acres are reported by three types of irrigation methods: sprinkler, microirrigation, and surface (flood) systems.

Irrigation withdrawals and irrigated acres by type of irrigation system are listed by State in table 7. For 2010, total irrigation withdrawals were $115,000 \mathrm{Mgal} / \mathrm{d}$, or 129,000 thousand acre-ft/yr, which accounted for 38 percent of total freshwater withdrawals and 61 percent of total freshwater withdrawals for all categories excluding thermoelectric power. Total irrigation withdrawals were 9 percent less than in 2005. Withdrawals from surface-water sources were $65,900 \mathrm{Mgal} / \mathrm{d}$, which accounted for 57 percent of the total irrigation withdrawals, and were almost 12 percent less than in 2005. Groundwater withdrawals for 2010 were 49,500 Mgal/d, or 6 percent less than in 2005 .

About 62,400 thousand acres were irrigated in 2010, an increase of about 950 thousand acres (1.5 percent) from 2005. About 31,600 thousand acres (51 percent) were irrigated with sprinkler systems, 26,200 thousand acres with surface (flood), and 4,610 thousand acres with microirrigation systems. The national average application rate for 2010 was 2.07 acrefeet per acre, or 11 percent less than the 2005 average of 2.32 acre-feet per acre.

The geographic distribution of total, surface-water, and groundwater withdrawals for irrigation is shown in figure 7. The majority of total U.S. irrigation withdrawals (83 percent) and irrigated acres ( 74 percent) were in the 17 conterminous Western States (west of solid line in figure 7), which are typical of areas where average annual precipitation is less than 20 inches and generally insufficient to support crops without supplemental water. Surface water was the primary source of water in the arid West, except in Kansas, Oklahoma, Nebraska, Texas, and South Dakota, where more groundwater was used. The 17 Western States cumulatively accounted for 93 percent of total surface-water irrigation withdrawals and 69 percent of total groundwater irrigation withdrawals.

Because the 17 Western States accounted for the majority of total irrigation withdrawals, changes in those States had a great effect on the overall total. Total irrigation withdrawals declined noticeably in Nebraska, Montana, Idaho, Colorado, and California. Groundwater irrigation withdrawals declined in the West and increased in the East, and surface-water irrigation withdrawals declined in both regions. Total irrigated acres increased in both regions - 1 percent (568 thousand acres) in the West, and 2 percent (381 thousand acres) in the East. In the West, the total number of acres irrigated by the less-efficient surface-irrigation methods decreased by about 500 thousand acres, and the number of acres irrigated by more efficient sprinkler (including microirrigation) methods increased by about 1,080 thousand acres.

Average application rates are calculated as a function of total irrigation withdrawals and total irrigated acres. The highest application rates are found in arid Western States, where more surface water than groundwater is used for irrigation and water typically is conveyed longer distances in canals between the points of diversion and use. Among the Western States, cumulatively, more lands were irrigated with sprinkler (including microirrigation) systems than surface methods, and land using the microirrigation system are increasing at a faster rate than the other two types of systems. Several States that used the large quantities of water for irrigation in 2010, such as California, Idaho, Colorado, Texas, and Nebraska, showed declines in application rates from 2005 levels, and in all of these States the number of acres irrigated by sprinkler or microirrigation systems increased in 2010.

Sources of data for irrigation withdrawals and irrigated acres included State and Federal crop reporting programs, irrigation districts, canal companies, incorporated management areas, and satellite data depicting 2010 cropland landscapes. Withdrawals also were estimated using information on irrigated crop acreages by crop type and specific crop water-consumption coefficients, or irrigation-system application rates, as well as soil moisture balance models. Estimation methods varied from one State to the next and sometimes between geographic areas within a State. Estimation methods ideally included adjustments for climate, system efficiencies, conveyance losses, and other irrigation practices such as pre-irrigation, salt leaching, or frost protection. Other methods for estimating irrigation withdrawals included extrapolation of sample data on crop water-application rates or power-consumption coefficients. 
Table 7. Irrigation water withdrawals, 2010.

[Values may not sum to totals because of independent rounding]

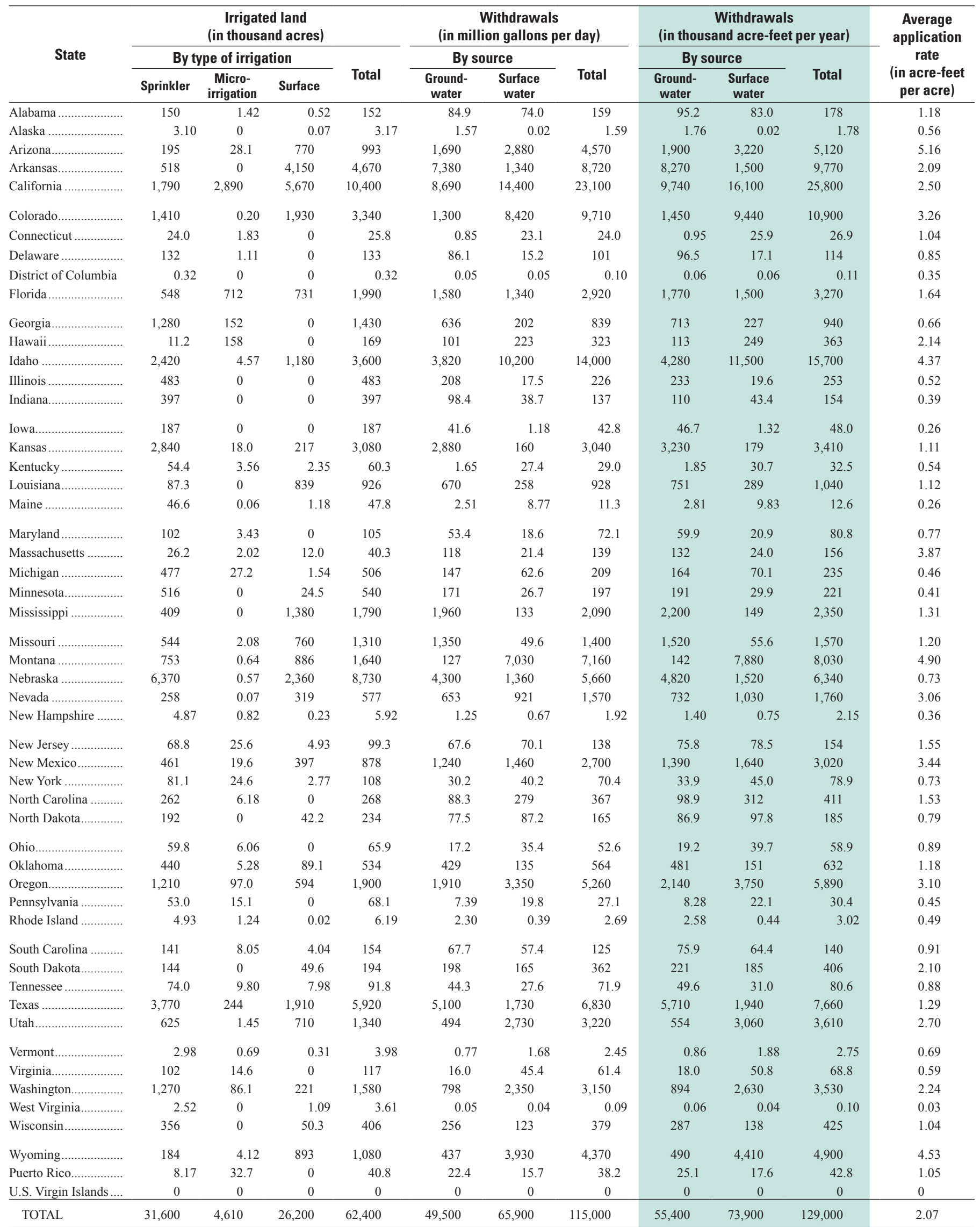



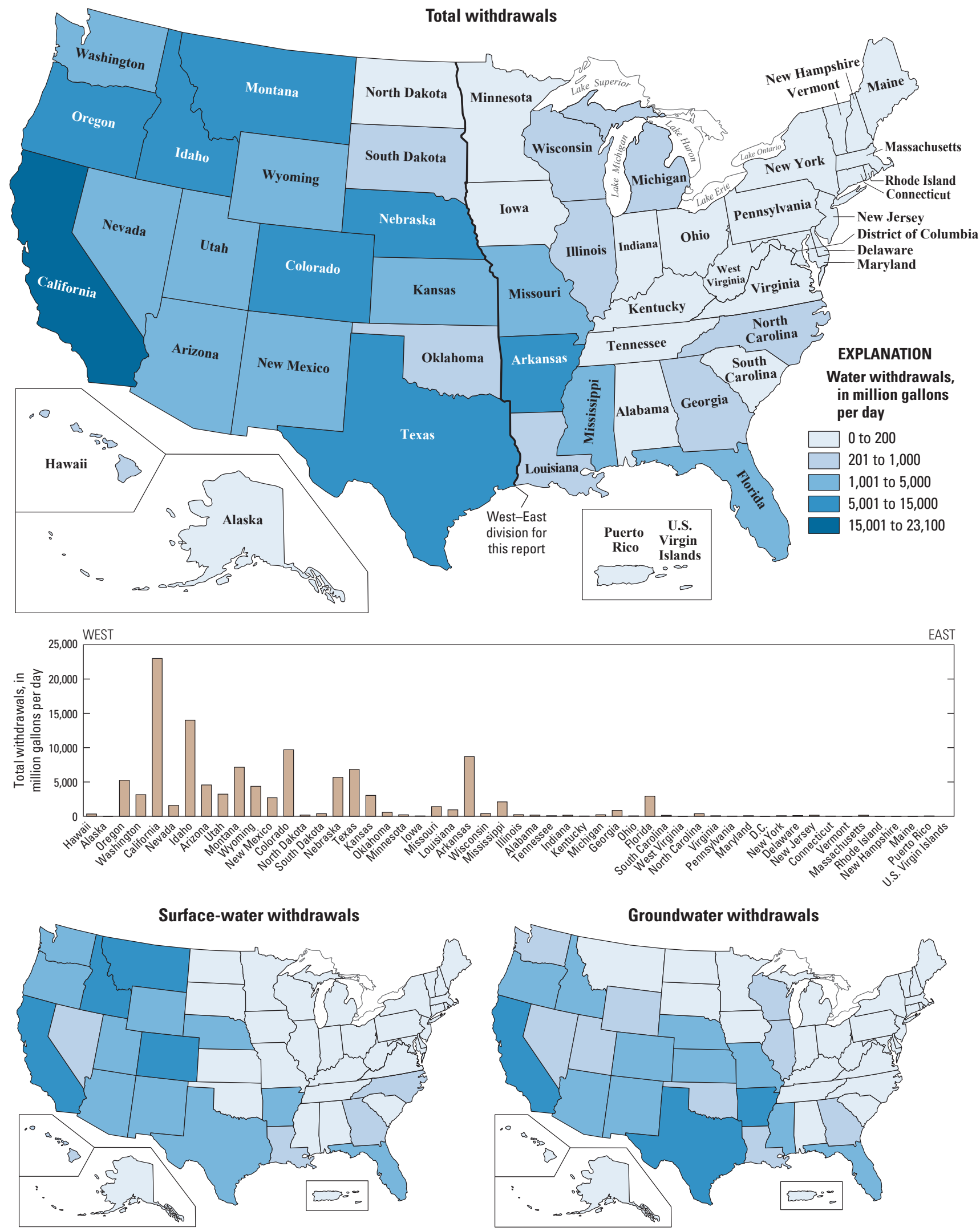

Figure 7. Irrigation withdrawals by source and State, 2010. 


\section{Livestock}

Livestock water use is water associated with livestock watering, feedlots, dairy operations, and other on-farm needs. Livestock includes dairy cows and heifers, beef cattle and calves, sheep and lambs, goats, hogs and pigs, horses, and poultry. Other livestock water uses include cooling of facilities for the animals and products, dairy sanitation and wash down of facilities, animal waste-disposal systems, and incidental water losses. All withdrawals were considered freshwater and self supplied. The livestock category excludes on-farm domestic use, lawn and garden watering, and irrigation water use.

Livestock withdrawals for 2010 are listed by State in table 8. During 2010, withdrawals for livestock use were an estimated 2,000 Mgal/d, or 2,240 thousand acre-ft/yr (table 2B). Livestock withdrawals were about 1 percent of total freshwater withdrawals and about 1 percent of total freshwater withdrawals for all categories excluding thermoelectric power. Groundwater was the source for 60 percent of total livestock withdrawals. Estimated total livestock withdrawals for 2010 were 7 percent less than in 2005 .

The geographic distribution of total, surface-water, and groundwater livestock withdrawals is shown in figure 8 . Texas, California, Iowa, Nebraska, and Kansas each used more than $100 \mathrm{Mgal} / \mathrm{d}$ for livestock and together accounted for 41 percent of total livestock withdrawals in 2010. Texas, Iowa, Nebraska, Kansas, and California each used more than $80 \mathrm{Mgal} / \mathrm{d}$ of groundwater for livestock and accounted for 42 percent of groundwater withdrawals for this use. Texas and California each used more than $100 \mathrm{Mgal} / \mathrm{d}$ of surface water for livestock, and accounted for 29 percent of surface-water withdrawals for livestock.

Few State agencies require livestock operations to report water withdrawals; therefore, most estimates of livestock withdrawals were derived using animal population data and water-use coefficients, in gallons per head per day for each animal type. Animal population data generally are available from State agricultural agencies and the NASS. Coefficients vary by State and, for many States, were provided by agricultural extension agents or water-permitting agencies. Coefficients may reflect facility maintenance needs and effects of climate on animal watering. Many of the 2010 withdrawals for livestock were estimated according to methods described by Lovelace (2009a), using livestock population data compiled for the NASS 2007 Census of Agriculture (Robert Hunt, National Agricultural Statistics Service, written commun., 2013) and water-use coefficients.

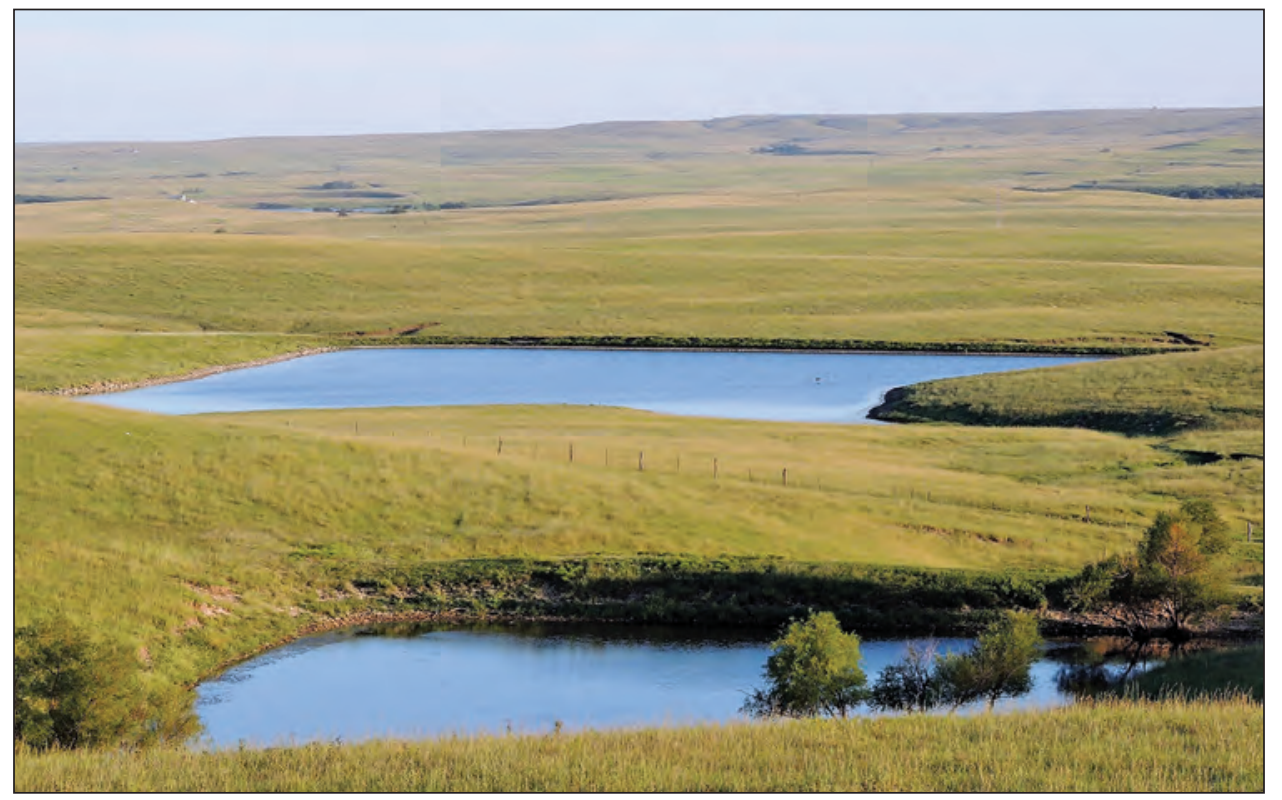

Stock ponds, Chase County, Kansas. Photo by Joan Kenny, USGS. 
Table 8. Livestock water withdrawals, 2010.

[Values may not sum to totals because of independent rounding]

\begin{tabular}{|c|c|c|c|}
\hline \multirow{3}{*}{ State } & \multicolumn{3}{|c|}{$\begin{array}{c}\text { Withdrawals } \\
\text { (in million gallons per day) }\end{array}$} \\
\hline & \multicolumn{2}{|c|}{ By source } & \multirow{2}{*}{ Total } \\
\hline & Groundwater & Surface water & \\
\hline Alabama .............................. & 11.7 & 14.8 & 26.5 \\
\hline Alaska ……........................ & 0.10 & 0.15 & 0.25 \\
\hline Arizona............................. & 27.0 & 0 & 27.0 \\
\hline Arkansas.......................... & 15.6 & 23.4 & 39.0 \\
\hline California ..................... & 84.4 & 103 & 188 \\
\hline Colorado............................ & 25.1 & 11.8 & 36.9 \\
\hline Connecticut ..................... & 1.01 & 0 & 1.01 \\
\hline Delaware ............................ & 1.31 & 0 & 1.31 \\
\hline District of Columbia & 0 & 0 & 0 \\
\hline 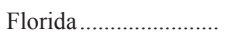 & 19.1 & 2.22 & 21.3 \\
\hline Georgia.............................. & 2.38 & 26.9 & 29.3 \\
\hline Hawaii ................................ & 0.63 & 1.20 & 1.83 \\
\hline Idaho …….................... & 38.5 & 9.01 & 47.5 \\
\hline Illinois .............................. & 36.0 & 0.03 & 36.1 \\
\hline Indiana.................................. & 26.2 & 13.0 & 39.2 \\
\hline Iowa & 102 & 33.8 & 136 \\
\hline Kansas ............................... & 91.0 & 23.0 & 114 \\
\hline Kentucky ......................... & 2.21 & 41.6 & 43.8 \\
\hline Louisiana......................... & 4.15 & 3.88 & 8.03 \\
\hline Maine …………................. & 1.71 & 0.58 & 2.29 \\
\hline Maryland ............................ & 6.02 & 2.23 & 8.25 \\
\hline Massachusetts .............. & 0.90 & 0.50 & 1.40 \\
\hline 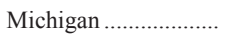 & 17.7 & 1.90 & 19.6 \\
\hline 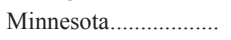 & 59.3 & 0 & 59.3 \\
\hline Mississippi ..................... & 7.35 & 11.1 & 18.4 \\
\hline Missouri …….................... & 18.4 & 54.4 & 72.9 \\
\hline Montana ............................ & 12.4 & 29.5 & 41.8 \\
\hline Nebraska ...................... & 93.0 & 21.2 & 114 \\
\hline Nevada ............................ & 5.06 & 0 & 5.06 \\
\hline New Hampshire ......... & 0.67 & 0.22 & 0.89 \\
\hline New Jersey ....................... & 0.98 & 0 & 0.98 \\
\hline New Mexico................. & 32.8 & 3.03 & 35.8 \\
\hline New York ....................... & 14.6 & 8.00 & 22.6 \\
\hline North Carolina ........... & 56.9 & 15.0 & 72.0 \\
\hline North Dakota.................. & 12.9 & 8.62 & 21.6 \\
\hline Ohio.................................... & 7.70 & 16.3 & 24.0 \\
\hline 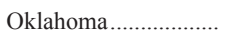 & 32.5 & 56.3 & 88.8 \\
\hline 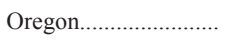 & 3.00 & 14.0 & 17.0 \\
\hline Pennsylvania .................. & 45.6 & 6.75 & 52.3 \\
\hline Rhode Island ..................... & 0.17 & 0.01 & 0.18 \\
\hline South Carolina ............. & 5.23 & 6.79 & 12.0 \\
\hline South Dakota................. & 19.1 & 28.3 & 47.4 \\
\hline Tennessee ...................... & 14.0 & 13.4 & 27.5 \\
\hline 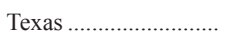 & 131 & 127 & 259 \\
\hline 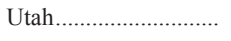 & 7.77 & 8.76 & 16.5 \\
\hline Vermont ............................ & 4.22 & 1.41 & 5.63 \\
\hline 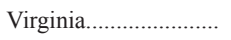 & 6.52 & 20.8 & 27.4 \\
\hline 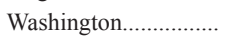 & 19.2 & 8.55 & 27.8 \\
\hline West Virginia................... & 1.66 & 3.42 & 5.08 \\
\hline Wisconsin ........................ & 65.8 & 7.30 & 73.1 \\
\hline 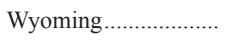 & 6.14 & 10.3 & 16.5 \\
\hline Puerto Rico.................... & 5.57 & 2.24 & 7.81 \\
\hline U.S. Virgin Islands .... & 0.01 & 0.01 & 0.02 \\
\hline TOTAL & 1,200 & 797 & 2,000 \\
\hline
\end{tabular}

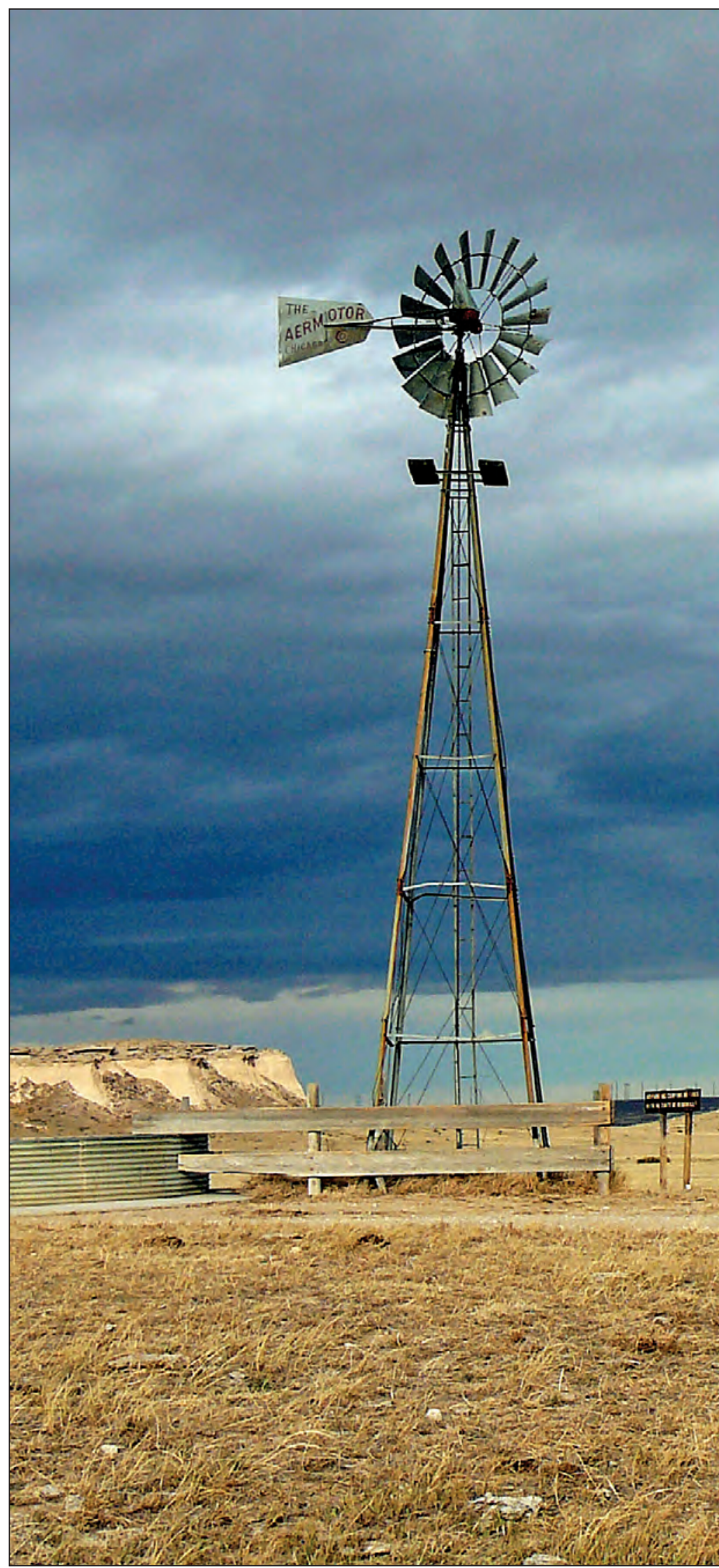

Windmill on Pawnee Butte Grasslands. Photo by Ray Klocek, used with permission. 

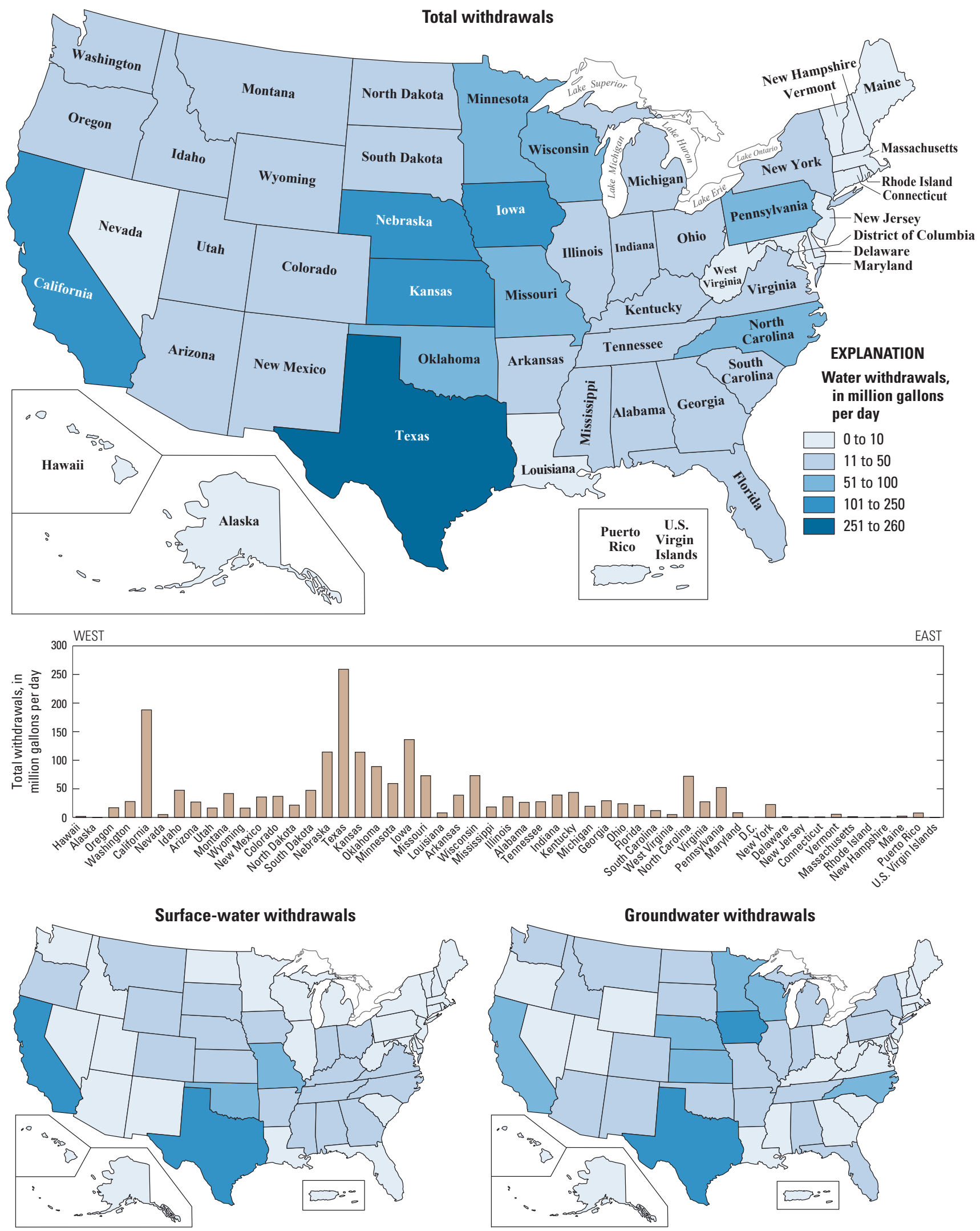

Figure 8. Livestock withdrawals by source and State, 2010. 
Aquaculture water use is water associated with raising organisms that live in water - such as finfish and shellfishfor food, restoration, conservation, or sport. Aquaculture production occurs under controlled feeding, sanitation, and harvesting procedures primarily in ponds, flowthrough raceways, and, to a lesser extent, cages, net pens, and closedrecirculation tanks. All withdrawals were considered self supplied.

Total withdrawals for aquaculture during 2010 are listed by State in table 9 at 9,420 Mgal/d, or 10,600 thousand acre-ft/yr (table 2B). Surface water was the source for about 81 percent of the withdrawals for this category. Much of the surface water was used for flowthrough raceways and was returned to the source after use. A combined total of 14.2 Mgal/d saline surface-water withdrawals, less than 0.2 percent of total aquaculture withdrawals, were reported in Rhode Island $(8.80 \mathrm{Mgal} / \mathrm{d})$ and Texas $(5.37 \mathrm{Mgal} / \mathrm{d})$; these amounts are not shown separately in table 9 but are included in the total. Aquaculture withdrawals were 3 percent of total withdrawals and 5 percent of total withdrawals for all categories excluding thermoelectric power. Estimated aquaculture withdrawals in 2010 were 7 percent more than in 2005.

The geographic distribution of total, surface-water, and groundwater withdrawals for aquaculture is shown in figure 9 . Idaho, North Carolina, California, and Oregon used the most water for aquaculture, about 63 percent of the total and about 74 percent of the surface-water withdrawals for aquaculture. Alaska, Louisiana, Arkansas, California, and Mississippi combined accounted for 60 percent of the total groundwater withdrawals for aquaculture.
Several sources of information were used to estimate 2010 aquaculture withdrawals. Some estimates were derived from State permits that reported water withdrawals or return flows for aquaculture facilities. The EPA Permit Compliance System database also was a source of return-flow data that were used to estimate water withdrawals. Individual aquaculture operations, State regulatory agencies, State offices of the NASS, and Cooperative Extension Service offices also provided information that was used to estimate aquaculture withdrawals in some States.

Many of the 2010 withdrawals for aquaculture were estimated by multiplying the number of aquaculture farms in operation in each county during 2007 by the average groundwater and surface-water withdrawal rates for aquaculture farms in the county. For the purpose of these estimates, the change in the number of aquaculture farms in each county from 2002 to 2007 was assumed to be representative of withdrawal changes from 2005 to 2010. The average groundwater and surface-water withdrawal rates for each county were calculated by dividing the estimated groundwater and surfacewater withdrawals for aquaculture in 2005 in the county by the number of aquaculture farms in operation in the county in 2002. The numbers of aquaculture farms in operation in 2002 and 2007 in each county were provided by the NASS (Robert Hunt, National Agricultural Statistics Service, written commun., 2013). In counties where no aquaculture operations existed in 2002, but one or more farms existed in 2007, the State average groundwater and surface-water withdrawal rates per farm were multiplied by the number of farms in the county.

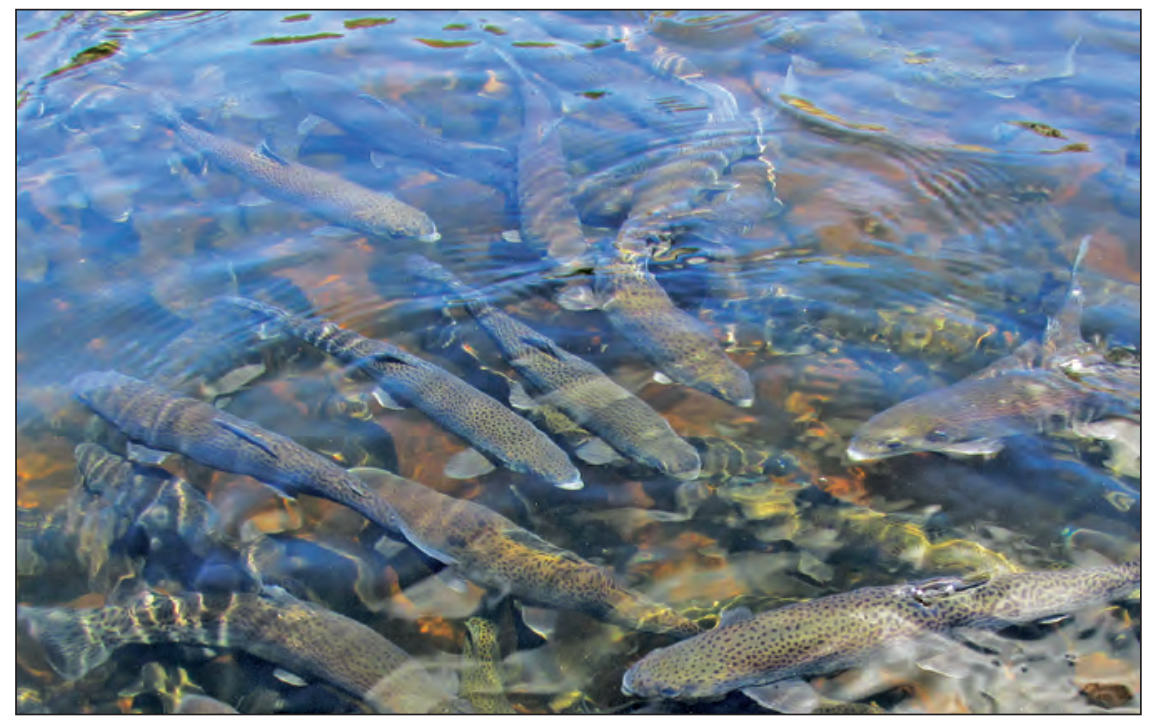

Left, trout in raceways at the historic Leadville National Fish Hatchery, Leadville, Colorado. Photo by Christopher Brown, USGS. Above, student with trout at the Aquaculture Research Institute in Hagerman, Idaho. Photo from University of Idaho, used with permission. 


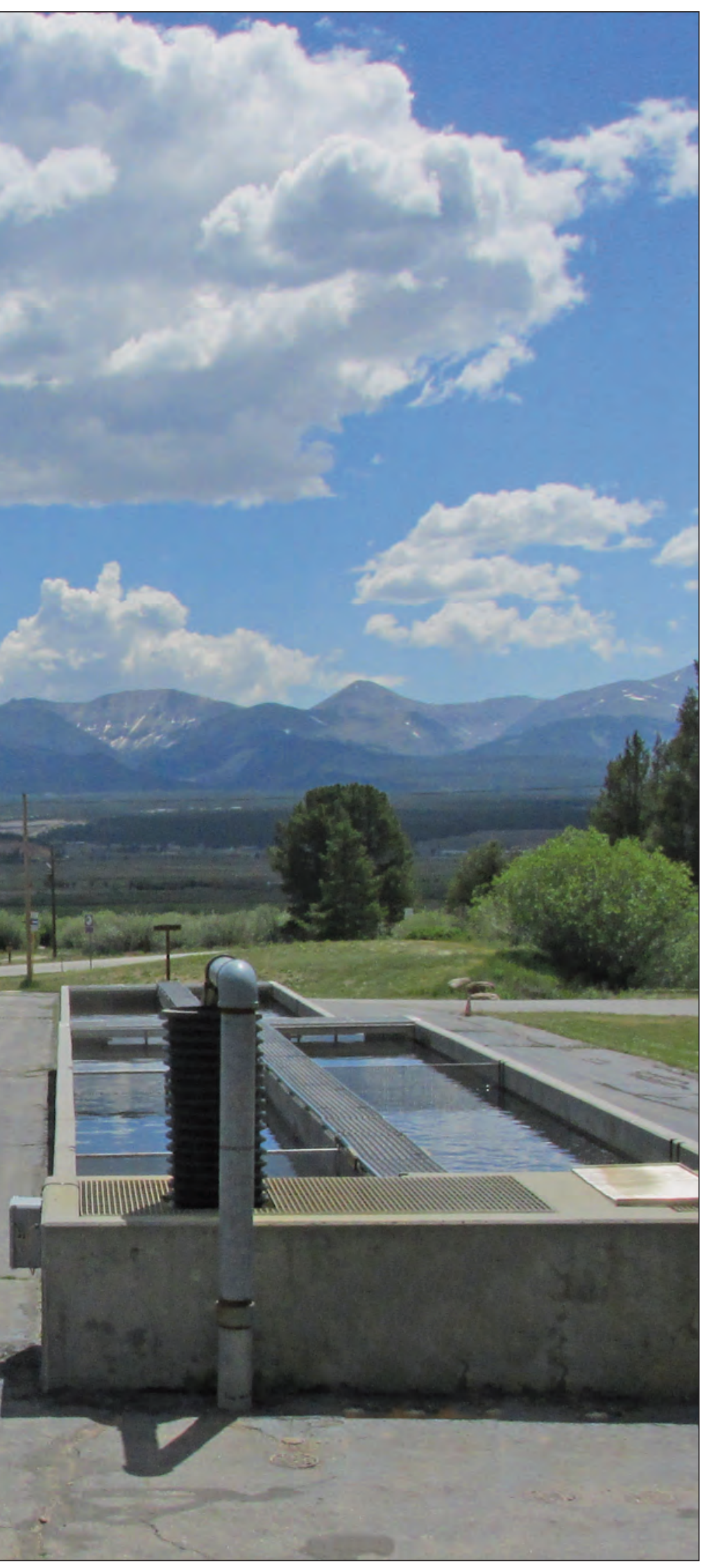

Raceway at historic Leadville National Fish Hatchery, Leadville, Colorado. Photo by Christopher Brown, USGS.
Table 9. Aquaculture water withdrawals, 2010.

[Values may not sum to totals because of independent rounding]

\begin{tabular}{|c|c|c|c|}
\hline \multirow{3}{*}{ State } & \multicolumn{3}{|c|}{$\begin{array}{c}\text { Withdrawals } \\
\text { (in million gallons per day) }\end{array}$} \\
\hline & \multicolumn{2}{|c|}{ By source } & \multirow{2}{*}{ Total } \\
\hline & Groundwater & Surface water & \\
\hline Alabama ........................ & 32.4 & 26.6 & 59.1 \\
\hline Alaska ............................ & 429 & 255 & 684 \\
\hline Arizona........................... & 39.5 & 7.77 & 47.3 \\
\hline Arkansas......................... & 181 & 86.5 & 268 \\
\hline California ....................... & 171 & 802 & 973 \\
\hline Colorado......................... & 23.0 & 99.0 & 122 \\
\hline Connecticut .................... & 6.67 & 23.0 & 29.7 \\
\hline Delaware ......................... & 0.06 & 0 & 0.06 \\
\hline District of Columbia & 0 & 0 & 0 \\
\hline 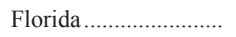 & 1.86 & 0 & 1.86 \\
\hline Georgia........................... & 3.92 & 45.9 & 49.8 \\
\hline Hawaii ............................ & 2.14 & 2.40 & 4.54 \\
\hline 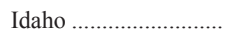 & 65.6 & 2,690 & 2,750 \\
\hline Illinois ............................ & 4.78 & 27.2 & 32.0 \\
\hline Indiana............................. & 6.60 & 1.97 & 8.57 \\
\hline Iowa............................... & 14.4 & 4.45 & 18.9 \\
\hline 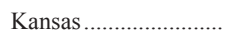 & 4.37 & 8.57 & 12.9 \\
\hline Kentucky ........................ & 0.53 & 33.5 & 34.1 \\
\hline Louisiana....................... & 197 & 114 & 311 \\
\hline Maine ........................... & 25.8 & 21.2 & 46.9 \\
\hline Maryland ......................... & 5.06 & 15.7 & 20.8 \\
\hline Massachusetts ............ & 7.23 & 42.4 & 49.6 \\
\hline Michigan ..................... & 4.21 & 78.5 & 82.7 \\
\hline Minnesota....................... & 1.69 & 15.2 & 16.9 \\
\hline Mississippi ................. & 113 & 19.3 & 133 \\
\hline Missouri ......................... & 10.5 & 170 & 181 \\
\hline Montana ......................... & 2.45 & 16.4 & 18.9 \\
\hline Nebraska ........................ & 6.07 & 82.2 & 88.3 \\
\hline Nevada ........................... & 10.6 & 38.8 & 49.5 \\
\hline New Hampshire ......... & 8.09 & 8.48 & 16.6 \\
\hline New Jersey ................... & 9.16 & 0 & 9.16 \\
\hline New Mexico................... & 15.8 & 4.32 & 20.1 \\
\hline New York .................... & 3.36 & 36.8 & 40.2 \\
\hline North Carolina ........... & 11.5 & 1,450 & 1,470 \\
\hline North Dakota............... & 0 & 5.92 & 5.92 \\
\hline 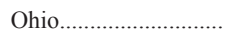 & 15.4 & 19.0 & 34.3 \\
\hline Oklahoma ........................ & 3.25 & 7.43 & 10.7 \\
\hline 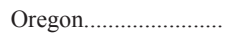 & 33.4 & 679 & 712 \\
\hline Pennsylvania ............... & 47.9 & 59.7 & 108 \\
\hline Rhode Island ............... & 5.60 & 8.90 & 14.5 \\
\hline South Carolina ........... & 2.00 & 8.97 & 11.0 \\
\hline South Dakota............... & 24.8 & 23.6 & 48.4 \\
\hline Tennessee ....................... & 15.4 & 37.2 & 52.6 \\
\hline Texas .............................. & 9.13 & 22.2 & 31.4 \\
\hline 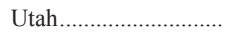 & 97.1 & 0 & 97.1 \\
\hline Vermont ......................... & 5.97 & 4.96 & 10.9 \\
\hline Virginia.......................... & 9.39 & 286 & 295 \\
\hline Washington...................... & 86.4 & 127 & 213 \\
\hline West Virginia............... & 11.7 & 40.6 & 52.3 \\
\hline Wisconsin ..................... & 25.5 & 30.2 & 55.8 \\
\hline Wyoming........................ & 2.10 & 18.7 & 20.8 \\
\hline Puerto Rico.................. & 0.01 & 0.40 & 0.41 \\
\hline U.S. Virgin Islands .... & 0 & 0 & 0 \\
\hline TOTAL & 1,820 & 7,610 & 9,420 \\
\hline
\end{tabular}



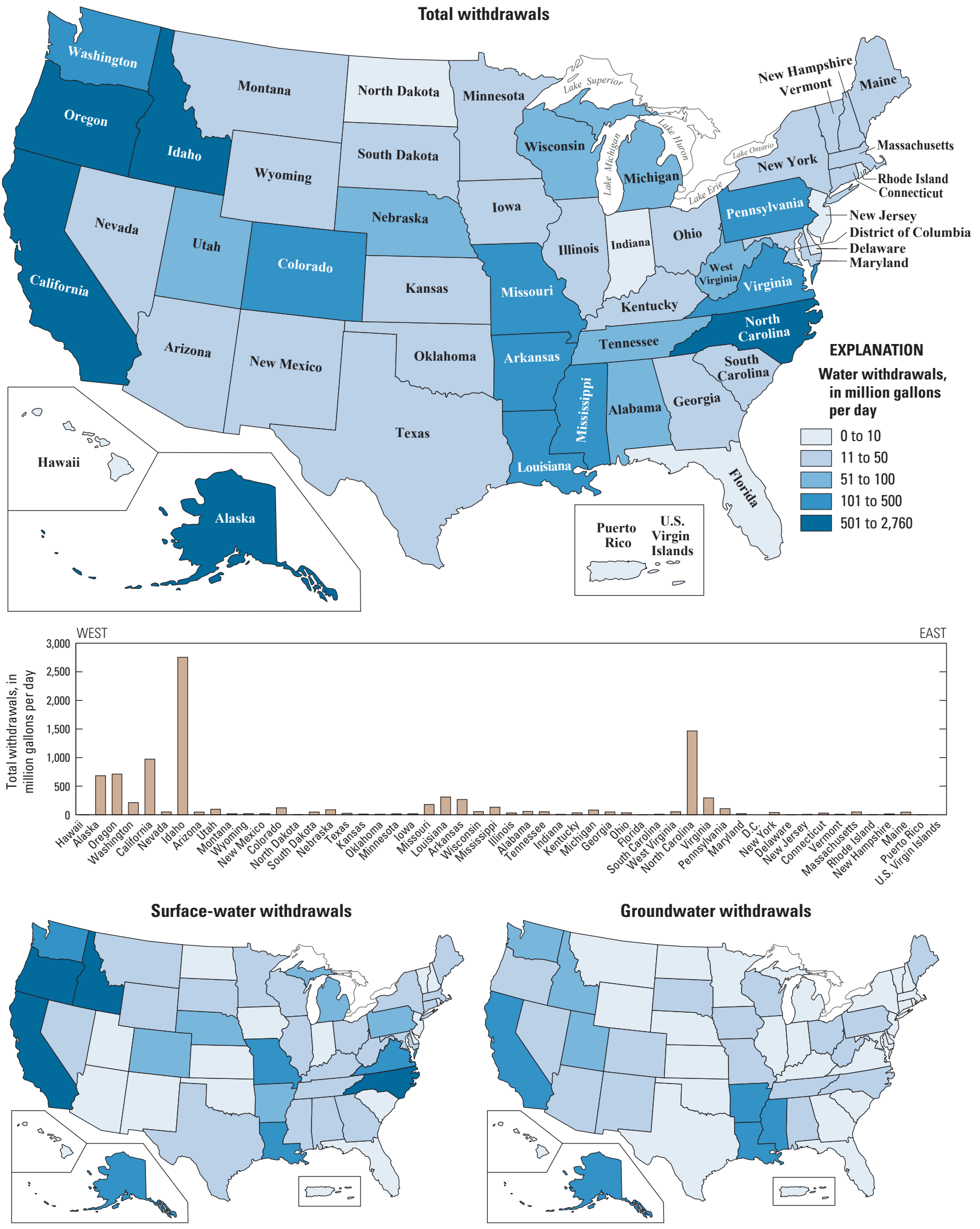

Figure 9. Aquaculture withdrawals by source and State, 2010. 
Industrial withdrawals provide water for such purposes as fabricating, processing, washing, diluting, cooling, or transporting a product; incorporating water into a product; or for sanitation needs within the manufacturing facility. Some industries that use large amounts of water produce such commodities as food, paper, chemicals, refined petroleum, or primary metals. Water for industrial use may be delivered from a public supplier or be self supplied. In this report, industrial use refers to self-supplied industrial withdrawals only. Withdrawals were reported as freshwater or saline water. As in the 2000 and 2005 reports, public-supply deliveries for industrial and consumptive uses were not reported for 2010 .

Industrial withdrawals are listed by State in table 10 . For 2010, withdrawals were an estimated 15,900 Mgal/d, or 17,900 thousand acre-ft/yr (table $2 B$ ). Industrial withdrawals were about 4 percent of total withdrawals and about 8 percent of total withdrawals for all categories excluding thermoelectric power. Surface water was the source for 82 percent of total industrial withdrawals, and 93 percent of the surfacewater withdrawals for industrial use was freshwater. More than 98 percent of the groundwater withdrawals for industrial use also was freshwater. For 2010, total industrial withdrawals were 12 percent less than in 2005.
The geographic distribution of total, total surface-water, and total groundwater withdrawals for industrial use is shown in figure 10. Indiana, Louisiana, and Texas accounted for 35 percent of total industrial withdrawals, and Indiana and Louisiana accounted for 33 percent of the total fresh surfacewater withdrawals. Texas accounted for 65 percent of the saline surface-water industrial withdrawals, mostly from areas along the Gulf coast. The largest fresh groundwater industrial withdrawals were in California, which accounted for 14 percent of the total national fresh groundwater industrial withdrawals. Most of the saline groundwater industrial withdrawals were in Utah.

Sources of data for industrial withdrawals included information obtained directly from facilities or State and Federal permit programs that require reporting of industrial withdrawals or return flows. Industrial withdrawals also were estimated using industry-group employment data and per employee water-use coefficients. A notable improvement from historical estimation methods include additional facility information provided to each USGS Water Science Center that included information about the type of business, number of employees, the location of the facilities, as well as economic indicators of the size of the business. These data were derived from a commercial database and were kept confidential.

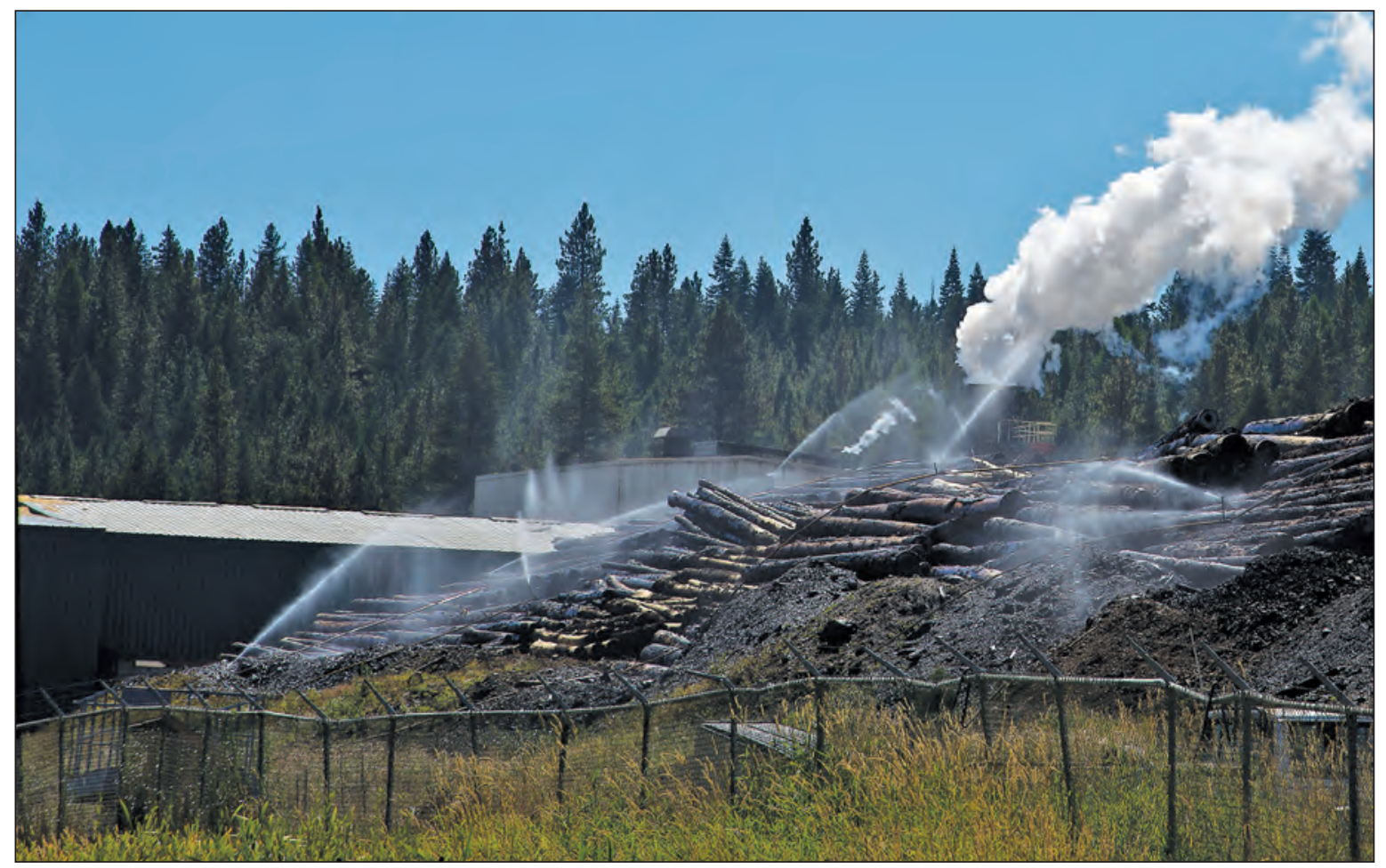

Lumber mill, Adams County, Idaho. Photo by Molly Maupin, USGS. 
Table 10. Industrial self-supplied water withdrawals, 2010.

[Values may not sum to totals because of independent rounding]

\begin{tabular}{|c|c|c|c|c|c|c|c|c|c|}
\hline \multirow{4}{*}{ State } & \multicolumn{9}{|c|}{ Withdrawals (in million gallons per day) } \\
\hline & \multicolumn{6}{|c|}{ By source and type } & \multirow{2}{*}{\multicolumn{3}{|c|}{ Total }} \\
\hline & \multicolumn{3}{|c|}{ Groundwater } & \multicolumn{3}{|c|}{ Surface water } & & & \\
\hline & Fresh & Saline & Total & Fresh & Saline & Total & Fresh & Saline & Total \\
\hline Alabama ........................... & 34.0 & 0 & 34.0 & 540 & 0 & 540 & 574 & 0 & 574 \\
\hline Alaska …………….......... & 3.38 & 0 & 3.38 & 4.40 & 4.30 & 8.70 & 7.78 & 4.30 & 12.1 \\
\hline Arizona............................ & 12.9 & 0 & 12.9 & 0 & 0 & 0 & 12.9 & 0 & 12.9 \\
\hline Arkansas.......................... & 56.1 & 5.05 & 61.2 & 214 & 0 & 214 & 271 & 5.05 & 276 \\
\hline California ...................... & 399 & 0 & 399 & 1.13 & 0 & 1.13 & 400 & 0 & 400 \\
\hline Colorado......................... & 3.45 & 0 & 3.45 & 127 & 0 & 127 & 130 & 0 & 130 \\
\hline Connecticut .................. & 6.28 & 0 & 6.28 & 60.2 & 38.5 & 98.6 & 66.5 & 38.5 & 105 \\
\hline Delaware .......................... & 8.43 & 0 & 8.43 & 87.5 & 0 & 87.5 & 96.0 & 0 & 96.0 \\
\hline District of Columbia & 0 & 0 & 0 & 0 & 0 & 0 & 0 & 0 & 0 \\
\hline Florida & 165 & 0 & 165 & 47.7 & 0 & 47.7 & 213 & 0 & 213 \\
\hline Georgia............................. & 206 & 0 & 206 & 281 & 0 & 281 & 487 & 0 & 487 \\
\hline 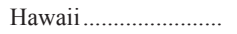 & 4.63 & 0 & 4.63 & 0 & 0 & 0 & 4.63 & 0 & 4.63 \\
\hline Idaho ………...................... & 32.6 & 0 & 32.6 & 17.2 & 0 & 17.2 & 49.7 & 0 & 49.7 \\
\hline Illinois ............................. & 124 & 0 & 124 & 267 & 0 & 267 & 390 & 0 & 390 \\
\hline Indiana.............................. & 82.2 & 0 & 82.2 & 2,120 & 0 & 2,120 & 2,210 & 0 & 2,210 \\
\hline Iowa & 123 & 0 & 123 & 2.70 & 0 & 2.70 & 125 & 0 & 125 \\
\hline Kansas ............................... & 33.5 & 0 & 33.5 & 6.79 & 0 & 6.79 & 40.3 & 0 & 40.3 \\
\hline Kentucky .......................... & 81.4 & 0 & 81.4 & 146 & 0 & 146 & 228 & 0 & 228 \\
\hline Louisiana......................... & 231 & 0 & 231 & 1,830 & 0 & 1,830 & 2,060 & 0 & 2,060 \\
\hline Maine …….......................... & 6.54 & 0 & 6.54 & 185 & 14.8 & 200 & 192 & 14.8 & 207 \\
\hline Maryland ......................... & 11.3 & 0 & 11.3 & 38.6 & 146 & 185 & 50.0 & 146 & 196 \\
\hline Massachusetts ............. & 4.28 & 0 & 4.28 & 12.1 & 0 & 12.1 & 16.3 & 0 & 16.3 \\
\hline Michigan ........................... & 75.0 & 0 & 75.0 & 537 & 0 & 537 & 612 & 0 & 612 \\
\hline Minnesota..................... & 61.8 & 0 & 61.8 & 71.7 & 0 & 71.7 & 134 & 0 & 134 \\
\hline Mississippi ....................... & 77.8 & 0 & 77.8 & 125 & 0 & 125 & 203 & 0 & 203 \\
\hline Missouri ............................ & 34.3 & 0 & 34.3 & 34.1 & 0 & 34.1 & 68.4 & 0 & 68.4 \\
\hline Montana ............................ & 36.9 & 0 & 36.9 & 29.6 & 0 & 29.6 & 66.4 & 0 & 66.4 \\
\hline Nebraska ............................ & 28.8 & 0 & 28.8 & 2.33 & 0 & 2.33 & 31.1 & 0 & 31.1 \\
\hline Nevada ….......................... & 0.70 & 0 & 0.70 & 4.53 & 0 & 4.53 & 5.23 & 0 & 5.23 \\
\hline New Hampshire ......... & 10.6 & 0 & 10.6 & 7.06 & 0 & 7.06 & 17.7 & 0 & 17.7 \\
\hline New Jersey .................... & 34.8 & 0 & 34.8 & 48.5 & 0 & 48.5 & 83.3 & 0 & 83.3 \\
\hline New Mexico ....................... & 10.3 & 0 & 10.3 & 0.83 & 0 & 0.83 & 11.1 & 0 & 11.1 \\
\hline New York .......................... & 35.9 & 0 & 35.9 & 316 & 0 & 316 & 352 & 0 & 352 \\
\hline North Carolina ............. & 83.8 & 0 & 83.8 & 188 & 0 & 188 & 271 & 0 & 271 \\
\hline North Dakota.................... & 5.77 & 0 & 5.77 & 12.9 & 0 & 12.9 & 18.7 & 0 & 18.7 \\
\hline 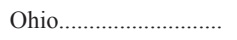 & 197 & 0 & 197 & 293 & 0 & 293 & 489 & 0 & 489 \\
\hline Oklahoma ........................ & 6.46 & 0 & 6.46 & 14.3 & 0 & 14.3 & 20.8 & 0 & 20.8 \\
\hline Oregon............................ & 2.62 & 0 & 2.62 & 123 & 0 & 123 & 126 & 0 & 126 \\
\hline Pennsylvania ............... & 73.8 & 0 & 73.8 & 792 & 0 & 792 & 866 & 0 & 866 \\
\hline Rhode Island ............... & 4.17 & 0 & 4.17 & 3.35 & 0 & 3.35 & 7.52 & 0 & 7.52 \\
\hline South Carolina ............. & 22.7 & 0 & 22.7 & 365 & 0 & 365 & 388 & 0 & 388 \\
\hline South Dakota................. & 6.85 & 0 & 6.85 & 2.63 & 0 & 2.63 & 9.48 & 0 & 9.48 \\
\hline Tennessee ..................... & 47.6 & 0 & 47.6 & 728 & 0 & 728 & 776 & 0 & 776 \\
\hline Texas ............................... & 108 & 2.04 & 110 & 571 & 608 & 1,180 & 680 & 610 & 1,290 \\
\hline Utah............................. & 31.2 & 37.5 & 68.7 & 16.4 & 33.1 & 49.5 & 47.6 & 70.6 & 118 \\
\hline Vermont............................ & 2.00 & 0 & 2.00 & 3.69 & 0 & 3.69 & 5.69 & 0 & 5.69 \\
\hline Virginia.......................... & 74.2 & 0.02 & 74.3 & 309 & 56.1 & 365 & 383 & 56.1 & 439 \\
\hline 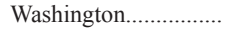 & 99.4 & 0 & 99.4 & 358 & 33.1 & 392 & 458 & 33.1 & 491 \\
\hline West Virginia............... & 35.1 & 3.80 & 38.9 & 729 & 0 & 729 & 764 & 3.80 & 768 \\
\hline Wisconsin ......................... & 54.3 & 0 & 54.3 & 382 & 0 & 382 & 436 & 0 & 436 \\
\hline Wyoming ........................... & 4.92 & 0 & 4.92 & 1.82 & 0 & 1.82 & 6.74 & 0 & 6.74 \\
\hline Puerto Rico....................... & 4.30 & 0 & 4.30 & 0 & 0 & 0 & 4.30 & 0 & 4.30 \\
\hline U.S. Virgin Islands .... & 0.22 & 0 & 0.22 & 0 & 2.62 & 2.62 & 0.22 & 2.62 & 2.84 \\
\hline TOTAL & 2,900 & 48.4 & 2,950 & 12,100 & 937 & 13,000 & 15,000 & 986 & 15,900 \\
\hline
\end{tabular}



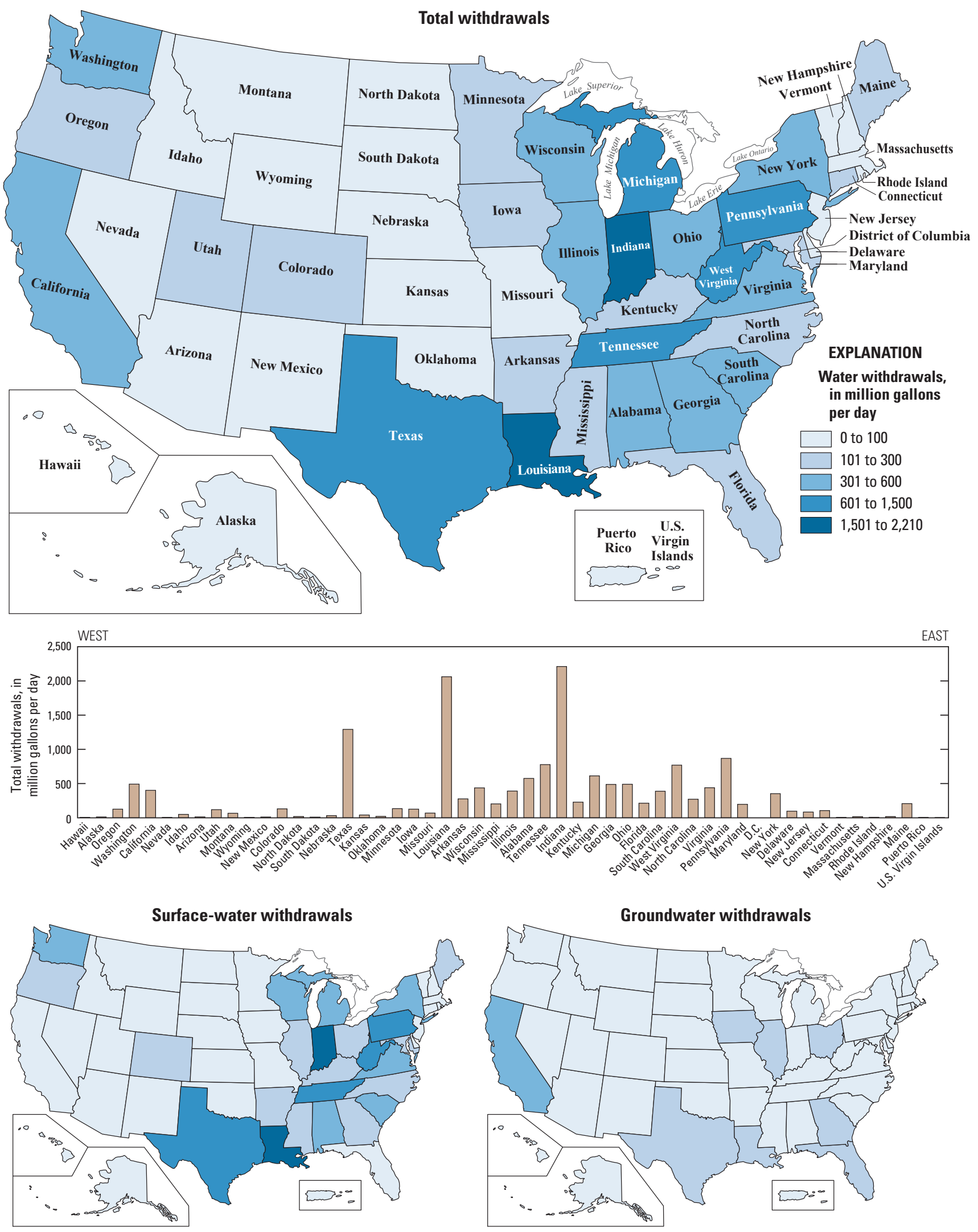

Figure 10. Industrial withdrawals by source and State, 2010. 
Mining water use is water used for the extraction of minerals that may be in the form of solids, such as coal, iron, sand, and gravel; liquids, such as crude petroleum; and gases, such as natural gas. The category includes quarrying, milling of mined materials, injection of water for secondary oil recovery or for unconventional oil and gas recovery (such as hydraulic fracturing), and other operations associated with mining activities. All mining withdrawals were considered to be self supplied. Water withdrawals were reported as freshwater or saline water. Dewatering was not reported as a mining withdrawal unless the water was used beneficially, such as dampening roads for dust control.

Mining withdrawals during 2010 are listed by State in table 11. During 2010, an estimated 5,320 Mgal/d, or 5,960 thousand acre-ft/yr (table 2B), were withdrawn. Mining withdrawals were about 1 percent of total withdrawals and about 3 percent of total withdrawals for all categories excluding thermoelectric power. Groundwater was the source for 73 percent of total withdrawals for mining. Seventy-one percent of the groundwater withdrawn for mining was saline. Eighty percent of the surface-water withdrawn was freshwater. Saline groundwater withdrawals and fresh surfacewater withdrawals together represented 74 percent of the total withdrawals for mining.

Total mining withdrawals in 2010 were 39 percent more than in 2005. Groundwater withdrawals were 54 percent more, and surface-water withdrawals were 9 percent more. Freshwater withdrawals in 2010 were only 1 percent less than in 2005, but saline-water withdrawals were 97 percent more than in 2005. Some of the increase in saline withdrawals was attributed to increased accounting of water produced as a byproduct during oil and gas extraction and then re-injected for secondary oil and gas recovery.

The geographic distribution of total, total freshwater, and total saline-water withdrawals is shown in figure 11. Oklahoma and Texas accounted for 46 percent of the total withdrawals for mining. Nevada and Texas accounted for 41 percent of fresh groundwater withdrawals, and Oklahoma and Texas accounted for 79 percent of saline groundwater withdrawals. Minnesota, Indiana, Texas, and Iowa accounted for 46 percent of fresh surface-water withdrawals. Utah and Alaska accounted for almost 100 percent of saline surfacewater withdrawals.

Sources of data used to estimate water use for mining included surveys of mining operations and State and Federal agencies that collect water withdrawal, discharge, or mineral production data for mining operations. Many of the 2010 withdrawals for mining were estimated according to methods described by Lovelace (2009b), using mineral production data and water-use coefficients, in gallons per weight or volume of minerals produced. Production data for nonfuel minerals, including metals and nonmetallic minerals, were provided by the USGS Minerals Information Team (Robert Callaghan, U.S. Geological Survey, written commun., 2012). Production or water-injection data for fuel minerals, including coal, petroleum, and natural gas, were obtained from the Energy Information Administration and various State agencies.

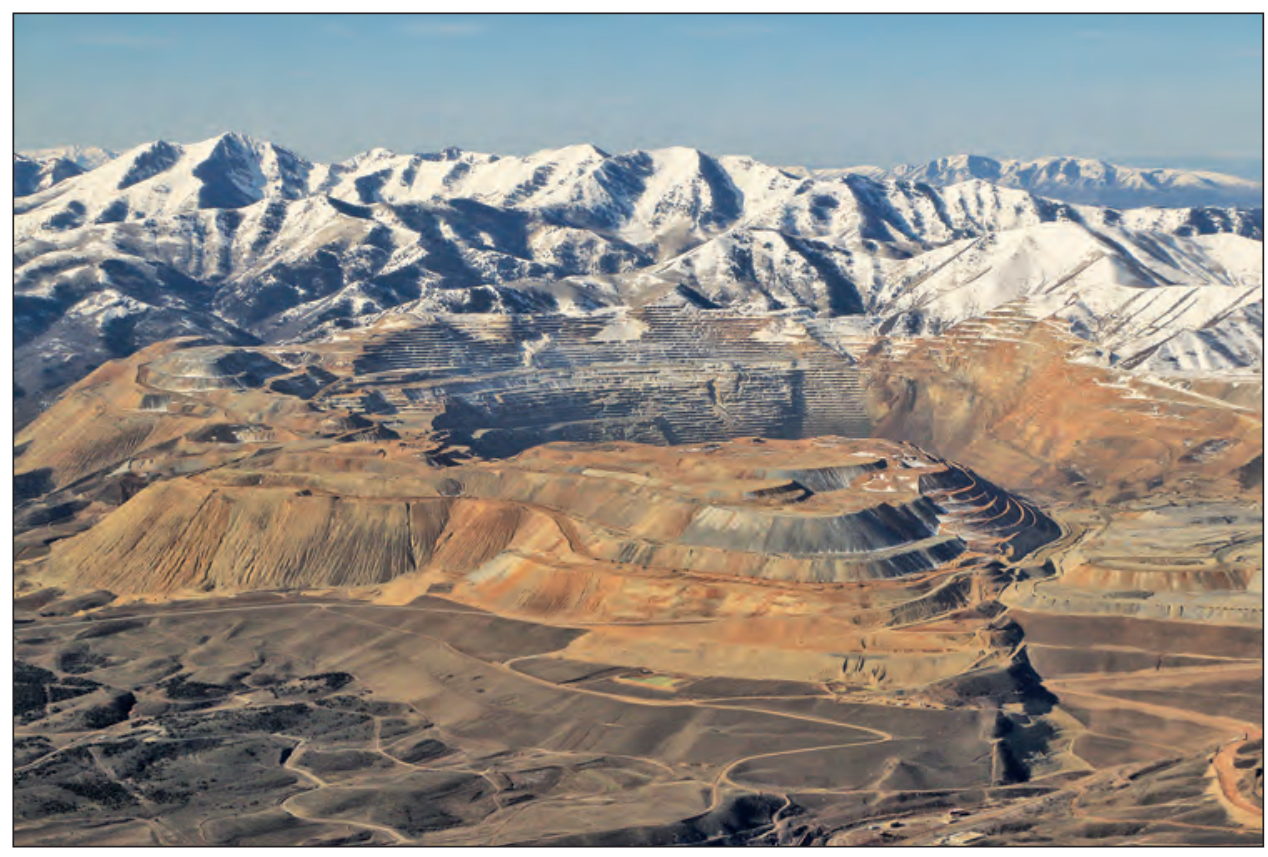

The Bingham Canyon Mine in Salt Lake County, Utah. Photo by Alan Cressler, USGS. 
Table 11. Mining water withdrawals, 2010.

[Values may not sum to totals because of independent rounding]

\begin{tabular}{|c|c|c|c|c|c|c|c|c|c|}
\hline \multirow{4}{*}{ State } & \multicolumn{9}{|c|}{ Withdrawals (in million gallons per day) } \\
\hline & \multicolumn{6}{|c|}{ By source and type } & \multirow{2}{*}{\multicolumn{3}{|c|}{ Total }} \\
\hline & \multicolumn{3}{|c|}{ Groundwater } & \multicolumn{3}{|c|}{ Surface water } & & & \\
\hline & Fresh & Saline & Total & Fresh & Saline & Total & Fresh & Saline & Total \\
\hline Alabama ........................... & 12.7 & 0 & 12.7 & 7.49 & 0 & 7.49 & 20.2 & 0 & 20.2 \\
\hline Alaska ............................. & 0.01 & 144 & 144 & 24.1 & 76.4 & 100 & 24.1 & 221 & 245 \\
\hline Arizona............................ & 86.6 & 0 & 86.6 & 0 & 0 & 0 & 86.6 & 0 & 86.6 \\
\hline Arkansas......................... & 0.18 & 0 & 0.18 & 44.1 & 0 & 44.1 & 44.3 & 0 & 44.3 \\
\hline California ...................... & 24.1 & 236 & 260 & 12.2 & 0.05 & 12.3 & 36.4 & 236 & 272 \\
\hline Colorado........................ & 5.46 & 19.4 & 24.9 & 3.05 & 0 & 3.05 & 8.51 & 19.4 & 27.9 \\
\hline Connecticut .................... & 0.92 & 0 & 0.92 & 3.80 & 0 & 3.80 & 4.72 & 0 & 4.72 \\
\hline Delaware .......................... & 0.44 & 0 & 0.44 & 0.41 & 0 & 0.41 & 0.85 & 0 & 0.85 \\
\hline District of Columbia & 0 & 0 & 0 & 0 & 0 & 0 & 0 & 0 & 0 \\
\hline Florida & 78.8 & 0 & 78.8 & 34.1 & 0 & 34.1 & 113 & 0 & 113 \\
\hline 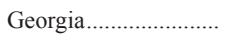 & 19.3 & 0 & 19.3 & 8.41 & 0 & 8.41 & 27.7 & 0 & 27.7 \\
\hline Hawaii ................................ & 1.40 & 0 & 1.40 & 0.11 & 0 & 0.11 & 1.51 & 0 & 1.51 \\
\hline Idaho ............................... & 1.28 & 0 & 1.28 & 18.9 & 0 & 18.9 & 20.2 & 0 & 20.2 \\
\hline Illinois .......................... & 15.5 & 25.5 & 41.0 & 55.4 & 0 & 55.4 & 70.9 & 25.5 & 96.3 \\
\hline Indiana........................... & 4.52 & 0 & 4.52 & 83.7 & 0 & 83.7 & 88.2 & 0 & 88.2 \\
\hline 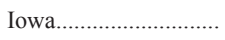 & 1.53 & 0 & 1.53 & 78.1 & 0 & 78.1 & 79.6 & 0 & 79.6 \\
\hline Kansas ............................... & 9.34 & 0 & 9.34 & 3.98 & 0 & 3.98 & 13.3 & 0 & 13.3 \\
\hline Kentucky .......................... & 7.80 & 0 & 7.80 & 23.0 & 0 & 23.0 & 30.8 & 0 & 30.8 \\
\hline Louisiana ........................... & 5.32 & 0 & 5.32 & 5.94 & 0 & 5.94 & 11.3 & 0 & 11.3 \\
\hline 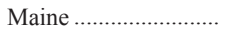 & 1.14 & 0 & 1.14 & 3.73 & 0 & 3.73 & 4.87 & 0 & 4.87 \\
\hline Maryland ........................... & 7.25 & 0 & 7.25 & 2.18 & 0 & 2.18 & 9.43 & 0 & 9.43 \\
\hline Massachusetts ............. & 1.82 & 0 & 1.82 & 4.78 & 0 & 4.78 & 6.60 & 0 & 6.60 \\
\hline Michigan .......................... & 10.1 & 0.57 & 10.7 & 66.0 & 0 & 66.0 & 76.2 & 0.57 & 76.8 \\
\hline 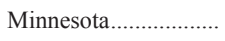 & 8.32 & 0 & 8.32 & 276 & 0 & 276 & 285 & 0 & 285 \\
\hline 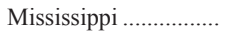 & 8.23 & 12.6 & 20.8 & 0.55 & 0 & 0.55 & 8.78 & 12.6 & 21.4 \\
\hline Missouri ............................ & 24.4 & 0 & 24.4 & 8.41 & 0 & 8.41 & 32.9 & 0 & 32.9 \\
\hline Montana & 1.73 & 18.6 & 20.3 & 26.2 & 0 & 26.2 & 27.9 & 18.6 & 46.5 \\
\hline Nebraska …………........... & 0.09 & 0.13 & 0.22 & 8.77 & 0 & 8.77 & 8.86 & 0.13 & 8.99 \\
\hline 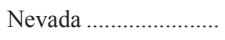 & 341 & 0.95 & 342 & 4.11 & 0 & 4.11 & 345 & 0.95 & 346 \\
\hline New Hampshire ......... & 0.01 & 0 & 0.01 & 2.84 & 0 & 2.84 & 2.85 & 0 & 2.85 \\
\hline New Jersey ...................... & 1.73 & 0 & 1.73 & 6.91 & 0 & 6.91 & 8.64 & 0 & 8.64 \\
\hline New Mexico.................... & 27.4 & 0 & 27.4 & 9.68 & 0 & 9.68 & 37.1 & 0 & 37.1 \\
\hline New York ......................... & 8.34 & 0 & 8.34 & 64.0 & 0 & 64.0 & 72.4 & 0 & 72.4 \\
\hline North Carolina ............ & 27.8 & 0 & 27.8 & 4.87 & 0 & 4.87 & 32.6 & 0 & 32.6 \\
\hline North Dakota................ & 8.73 & 13.6 & 22.3 & 4.63 & 0 & 4.63 & 13.4 & 13.6 & 27.0 \\
\hline 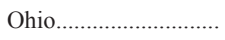 & 79.0 & 0 & 79.0 & 35.8 & 0 & 35.8 & 115 & 0 & 115 \\
\hline Oklahoma ....................... & 4.75 & 1,400 & 1,400 & 13.3 & 0 & 13.3 & 18.0 & 1,400 & 1,410 \\
\hline Oregon............................... & 7.47 & 0 & 7.47 & 1.17 & 0 & 1.17 & 8.64 & 0 & 8.64 \\
\hline Pennsylvania ................... & 51.4 & 0 & 51.4 & 10.5 & 0 & 10.5 & 62.0 & 0 & 62.0 \\
\hline Rhode Island .................. & 0.43 & 0 & 0.43 & 0.49 & 0 & 0.49 & 0.92 & 0 & 0.92 \\
\hline South Carolina ............ & 6.69 & 0 & 6.69 & 1.74 & 0 & 1.74 & 8.43 & 0 & 8.43 \\
\hline South Dakota................... & 7.22 & 0 & 7.22 & 11.0 & 0 & 11.0 & 18.2 & 0 & 18.2 \\
\hline Tennessee ....................... & 6.89 & 0 & 6.89 & 7.73 & 0 & 7.73 & 14.6 & 0 & 14.6 \\
\hline Texas .............................. & 122 & 810 & 931 & 81.2 & 0.49 & 81.7 & 203 & 810 & 1,010 \\
\hline Utah..................................... & 2.59 & 41.6 & 44.2 & 1.60 & 205 & 206 & 4.19 & 246 & 250 \\
\hline Vermont............................ & 0.32 & 0 & 0.32 & 3.53 & 0 & 3.53 & 3.85 & 0 & 3.85 \\
\hline 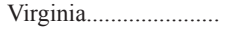 & 6.56 & 0 & 6.56 & 28.4 & 0 & 28.4 & 34.9 & 0 & 34.9 \\
\hline 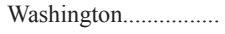 & 13.4 & 0 & 13.4 & 3.36 & 0 & 3.36 & 16.7 & 0 & 16.7 \\
\hline West Virginia............... & 5.53 & 1.02 & 6.55 & 9.00 & 0 & 9.00 & 14.5 & 1.02 & 15.6 \\
\hline 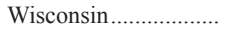 & 10.9 & 0 & 10.9 & 8.63 & 0 & 8.63 & 19.6 & 0 & 19.6 \\
\hline 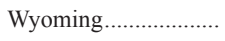 & 37.1 & 67.1 & 104 & 13.0 & 0 & 13.0 & 50.1 & 67.1 & 117 \\
\hline Puerto Rico...................... & 1.43 & 0.32 & 1.75 & 0.18 & 0 & 0.18 & 1.61 & 0.32 & 1.93 \\
\hline U.S. Virgin Islands .... & 0 & 0 & 0 & 0 & 0.04 & 0.04 & 0 & 0.04 & 0.04 \\
\hline TOTAL & 1,120 & 2,790 & 3,900 & 1,130 & 282 & 1,410 & 2,250 & 3,070 & 5,320 \\
\hline
\end{tabular}



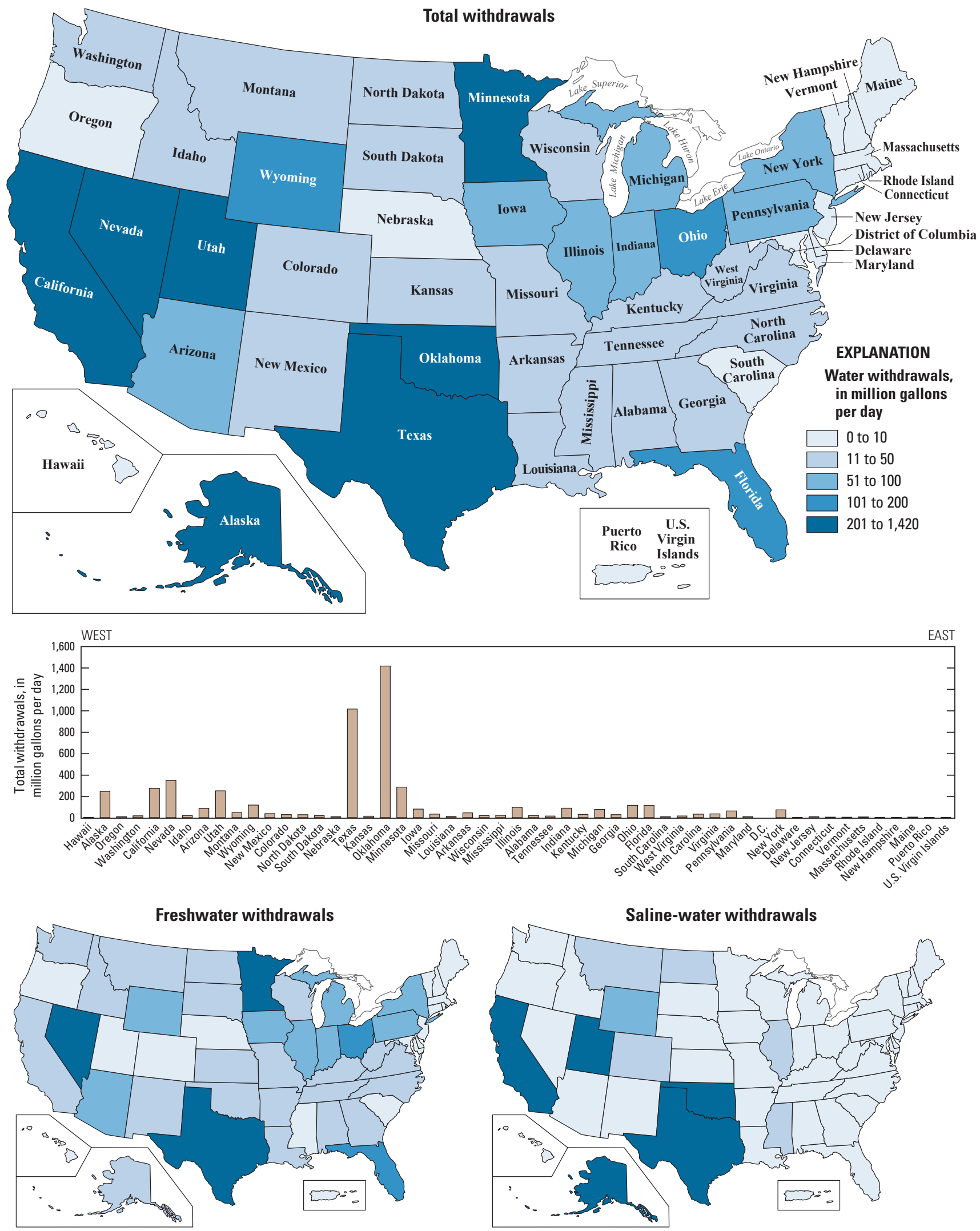

Figure 11. Mining withdrawals by water quality and State, 2010. 
Water for thermoelectric power is used in generating electricity with steam-driven turbine generators. Thermoelectricpower withdrawals were compiled by cooling-system type. Once-through cooling systems circulate water through heat exchangers and then return the water to the source. Recirculation cooling systems circulate water through heat exchangers, then cool the water using ponds or towers, and then the water is recirculated. Water withdrawals for a recirculation system are used to replace water lost to evaporation, blowdown, drift, and leakage. Thermoelectric-power withdrawals were reported as freshwater or saline water, as well as by cooling system. Net power generation is also reported by cooling system.

For 2010, public-supply deliveries to thermoelectric powerplants and consumptive use were not reported. However, 1,290 thermoelectric powerplants from the linked heat and water budget models provided monthly and annual estimates of withdrawals and consumptive use by powerplant. These data were provided as supplemental and supportive datasets for the compilation. Datasets substantially improved existing NWUIP capabilities with more accurate and complete information on thermoelectric powerplant locations, categorization of cooling-system types, and water sources. Qualityassured data for net power generation were also provided with linked information about cooling system and fuels, monthly and annual (2010) estimates of withdrawals based on the models, and associated monthly and annual estimates of consumptive use by plant (Diehl and others, 2013; Diehl and Harris, 2014). These data were used either in whole, or in part, for this compilation. Compilers in some States obtained data reported directly from thermoelectric powerplants.

Thermoelectric-power withdrawals and net power generation are listed by State in table 12. Total withdrawals for thermoelectric power for 2010 were $161,000 \mathrm{Mgal} / \mathrm{d}$ or 180,000 thousand acre-ft/yr (table $2 B$ ). Surface water was the source for over 99 percent of total thermoelectric-power withdrawals, and 73 percent of those surface-water withdrawals were from freshwater sources. Saline surface-water withdrawals for thermoelectric power accounted for 97 percent of total saline surface-water withdrawals for all uses. Total withdrawals for thermoelectric power accounted for 45 percent of total water withdrawals, 38 percent of total freshwater withdrawals, and 51 percent of fresh surface-water withdrawals for all uses. Net power generation associated with thermoelectric-power withdrawals was 3,130,000 $\mathrm{gWh}$ (gigawatt-hours), or about 2 percent less than in 2005. On average, 19 gal (gallons) were used to produce $1 \mathrm{kWh}$ (kilowatt-hour) of electricity in 2010, compared to almost $23 \mathrm{gal} / \mathrm{kWh}$ (gallons per kilowatt-hour) in 2005.

The geographic distribution of total, total freshwater, and total saline-water withdrawals for thermoelectric power is shown in figure 12. The largest total withdrawals for thermoelectric power were in Texas, where nearly all the withdrawals were from freshwater sources. Illinois, Texas,
Michigan, and Alabama, together accounted for more than 32 percent of freshwater withdrawals for thermoelectric power. Florida, California, and Maryland accounted for about 48 percent of total saline withdrawals, nearly all from surface water. Hawaii, California, and Nevada accounted for 82 percent of the total saline groundwater withdrawals.

Estimated 2010 thermoelectric withdrawals were 20 percent less than estimates for 2005. Reasons for this large difference include plant closures, use of the linked heat and water budget model data, decrease in use of coal and increase in use of natural gas, and new powerplants using more waterefficient cooling technology.

Eastern States accounted for 86 percent of total thermoelectric-power withdrawals in the United States and 75 percent of the related net power generation. Hydroelectricpower generation is not included in this report but meets the demand for a significant amount of the U.S. total energy needs, predominantly in Western States. In 2010, 61 percent of the total $257,000 \mathrm{gWh}$ from hydroelectric powerplants was produced by public utilities in Washington, California, Oregon, and New York (U.S. Department of Energy, 2011).

Thermoelectric-power withdrawals and net power generated by cooling-system type are listed by State in table 13. Powerplants with once-through cooling systems accounted for 94 percent of total withdrawals and 47 percent of net power generated. Plants with recirculating cooling systems required much less water ( 6 percent) and produced the majority (53 percent) of the net power generated. Powerplants with recirculating cooling systems are found in every State but were the predominant type of cooling system at powerplants in Western inland States such as Arizona, Oklahoma, Wyoming, Utah, Colorado, Kansas, and New Mexico.

Reclaimed wastewater is a supplemental source of water for thermoelectric power, especially in areas where additional water sources are needed for plant operations, such as for air pollution control equipment, or scrubbers (Veil, 2007). Arizona (67.6 Mgal/d) and California (22.8 Mgal/d) reported substantial amounts of reclaimed water use. Reclaimed wastewater is not included in the thermoelectric-power data or national totals for this report.

Sources for thermoelectric-power withdrawals, coolingsystem information, and net power generation included data collected directly from facilities, State permitting or regulatory agencies, the USDOE EIA, and a linked heat and water budget for powerplants in the United States, as mentioned previously. Using information gleaned from the NWC thermoelectric project, some powerplant's coolingsystem classifications were changed, thereby making them different from previous compilations. Similarly, net powergeneration data from EIA were scrutinized for each plant to determine whether the power that was reported and used in this compilation was associated with a water use. 
Table 12. Thermoelectric-power water withdrawals, 2010.

[Values may not sum to totals because of independent rounding]

\begin{tabular}{|c|c|c|c|c|c|c|c|c|c|c|}
\hline \multirow{3}{*}{ State } & \multicolumn{9}{|c|}{ Withdrawals (in million gallons per day) } & \multirow{3}{*}{$\begin{array}{c}\text { Power } \\
\text { generated } \\
\text { (in million } \\
\text { kilowatt-hours }\end{array}$} \\
\hline & \multicolumn{6}{|c|}{ By source and type } & \multicolumn{3}{|c|}{ Total } & \\
\hline & Fresh & Saline & Total & Fresh & Saline & Total & Fresh & Saline & Total & \\
\hline Alaska ............................ & 2.19 & 0 & 2.19 & 55.8 & 0 & 55.8 & 58.0 & 0 & 58.0 & 1,760 \\
\hline Arizona........................... & 77.3 & 0 & 77.3 & 27.1 & 0 & 27.1 & 104 & 0 & 104 & 86,700 \\
\hline Arkansas........................ & 4.26 & 0 & 4.26 & 1,540 & 0 & 1,540 & 1,540 & 0 & 1,540 & 49,000 \\
\hline California ..................... & 33.1 & 48.4 & 81.6 & 32.2 & 6,490 & 6,520 & 65.4 & 6,540 & 6,600 & 85,400 \\
\hline Connecticut ................. & 0 & 0 & 0 & 198 & 2,460 & 2,650 & 198 & 2,460 & 2,650 & 21,600 \\
\hline Delaware ...................... & 0.37 & 0 & 0.37 & 7.45 & 417 & 425 & 7.82 & 417 & 425 & 5,150 \\
\hline District of Columbia & 0 & 0 & 0 & 0 & 0 & 0 & 0 & 0 & 0 & 0 \\
\hline Florida ............................. & 43.5 & 6.54 & 50.0 & 570 & 8,570 & 9,140 & 613 & 8,570 & 9,190 & 199,000 \\
\hline 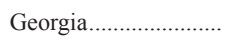 & 2.92 & 0 & 2.92 & 1,770 & 283 & 2,050 & 1,770 & 283 & 2,060 & 110,000 \\
\hline Hawaii .............................. & 53.2 & 50.8 & 104 & 0 & 552 & 552 & 53.2 & 603 & 656 & 6,830 \\
\hline Kansas ............................ & 11.2 & 0 & 11.2 & 366 & 0 & 366 & 377 & 0 & 377 & 43,600 \\
\hline Kentucky ........................ & 15.3 & 0 & 15.3 & 3,340 & 0 & 3,340 & 3,360 & 0 & 3,360 & 93,400 \\
\hline 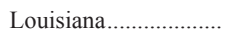 & 41.1 & 0 & 41.1 & 4,390 & 1.68 & 4,390 & 4,430 & 1.68 & 4,430 & 63,500 \\
\hline Maine .............................. & 0.96 & 0 & 0.96 & 25.9 & 26.0 & 51.9 & 26.8 & 26.0 & 52.8 & 7,650 \\
\hline Maryland ......................... & 2.25 & 0 & 2.25 & 434 & 5,760 & 6,200 & 436 & 5,760 & 6,200 & 41,100 \\
\hline Massachusetts ............ & 0.21 & 0 & 0.21 & 134 & 1,930 & 2,070 & 134 & 1,930 & 2,070 & 18,500 \\
\hline Michigan .......................... & 4.12 & 0 & 4.12 & 8,510 & 0 & 8,510 & 8,520 & 0 & 8,520 & 107,000 \\
\hline 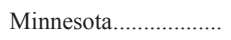 & 2.34 & 0 & 2.34 & 2,510 & 0 & 2,510 & 2,510 & 0 & 2,510 & 42,400 \\
\hline Mississippi ..................... & 50.0 & 7.05 & 57.1 & 905 & 62.4 & 968 & 956 & 69.5 & 1,020 & 44,200 \\
\hline 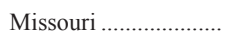 & 19.9 & 0 & 19.9 & 5,890 & 0 & 5,890 & 5,910 & 0 & 5,910 & 86,200 \\
\hline Montana ........................... & 0.85 & 0 & 0.85 & 150 & 0 & 150 & 151 & 0 & 151 & 19,000 \\
\hline Ohio.................................... & 23.0 & 0 & 23.0 & 7,190 & 0 & 7,190 & 7,220 & 0 & 7,220 & 137,000 \\
\hline 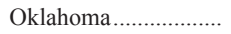 & 1.26 & 0 & 1.26 & 384 & 0 & 384 & 385 & 0 & 385 & 48,700 \\
\hline Oregon............................ & 1.48 & 0 & 1.48 & 11.2 & 0 & 11.2 & 12.7 & 0 & 12.7 & 8,820 \\
\hline Pennsylvania .................. & 4.49 & 0 & 4.49 & 5,390 & 0 & 5,390 & 5,390 & 0 & 5,390 & 201,000 \\
\hline Rhode Island .................. & 0 & 0 & 0 & 1.44 & 232 & 234 & 1.44 & 232 & 234 & 1,140 \\
\hline South Carolina ............ & 4.86 & 0 & 4.86 & 5,500 & 0 & 5,500 & 5,500 & 0 & 5,500 & 97,800 \\
\hline South Dakota................. & 3.34 & 0 & 3.34 & 6.93 & 0 & 6.93 & 10.3 & 0 & 10.3 & 3,300 \\
\hline Tennessee ...................... & 1.78 & 0 & 1.78 & 5,800 & 0 & 5,800 & 5,800 & 0 & 5,800 & 71,900 \\
\hline 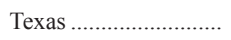 & 38.8 & 0 & 38.8 & 10,400 & 661 & 11,100 & 10,500 & 661 & 11,100 & 249,000 \\
\hline Utah............................... & 24.0 & 10.5 & 34.5 & 45.6 & 0.47 & 46.0 & 69.6 & 11.0 & 80.6 & 38,900 \\
\hline 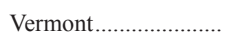 & 0.74 & 0 & 0.74 & 344 & 0 & 344 & 345 & 0 & 345 & 5,230 \\
\hline Virginia............................... & 1.55 & 0 & 1.55 & 2,850 & 3,150 & 6,000 & 2,860 & 3,150 & 6,000 & 53,500 \\
\hline 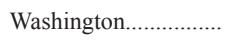 & 1.57 & 0 & 1.57 & 36.4 & 0 & 36.4 & 37.9 & 0 & 37.9 & 20,100 \\
\hline West Virginia.................. & 1.40 & 0 & 1.40 & 2,470 & 0 & 2,470 & 2,470 & 0 & 2,470 & 77,500 \\
\hline Wisconsin ....................... & 2.78 & 0 & 2.78 & 4,630 & 0 & 4,630 & 4,630 & 0 & 4,630 & 55,400 \\
\hline 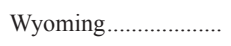 & 2.29 & 0 & 2.29 & 61.1 & 0 & 61.1 & 63.4 & 0 & 63.4 & 39,600 \\
\hline Puerto Rico.................... & 1.17 & 0 & 1.17 & 2.61 & 2,270 & 2,270 & 3.78 & 2,270 & 2,270 & 19,900 \\
\hline U.S. Virgin Islands .... & 0 & 0 & 0 & 0.17 & 116 & 116 & 0.17 & 116 & 116 & 830 \\
\hline TOTAL & 587 & 134 & 721 & 116,000 & 43,800 & 160,000 & 117,000 & 43,900 & 161,000 & $3,130,000$ \\
\hline
\end{tabular}



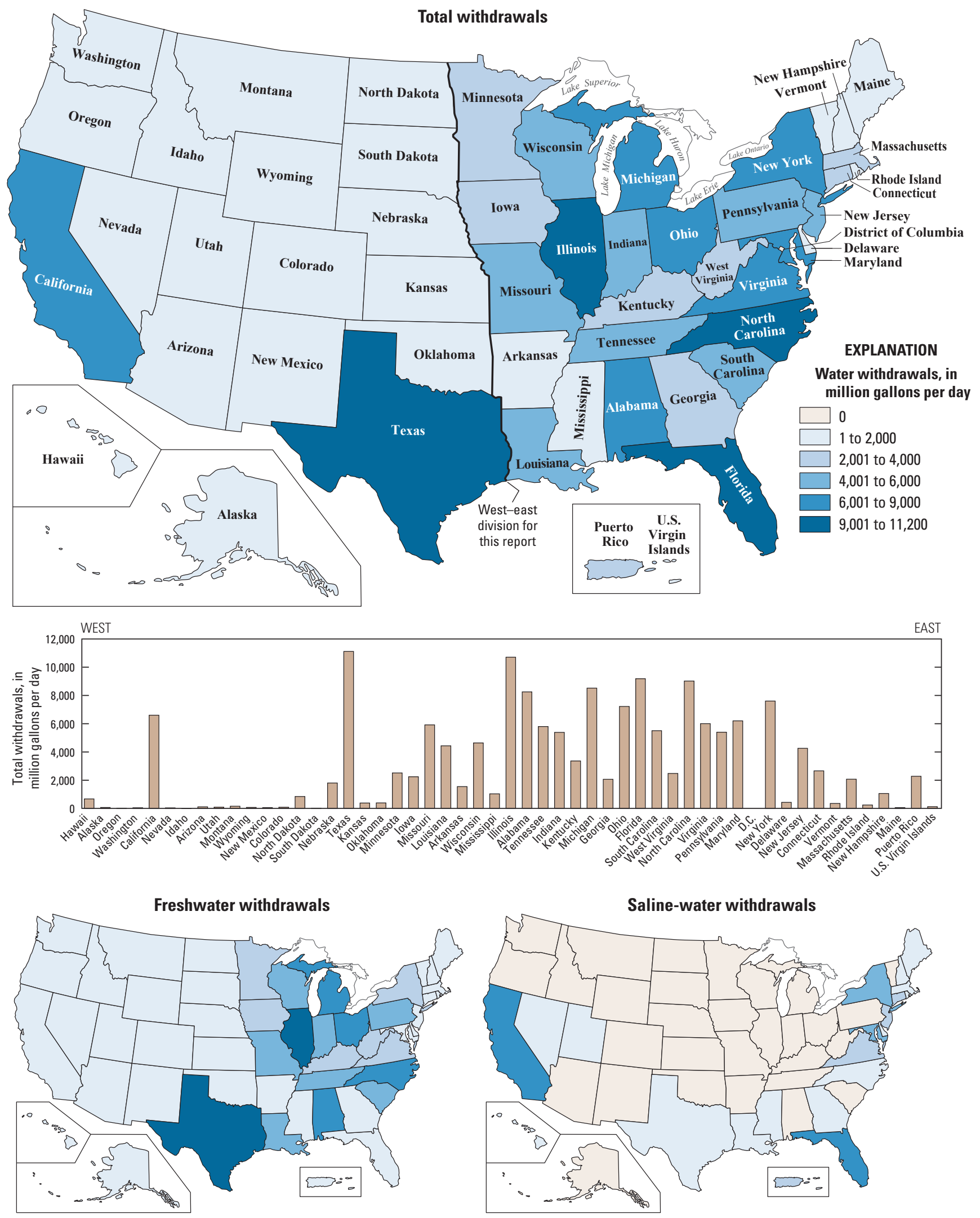

Figure 12. Thermoelectric-power withdrawals by water quality and State, 2010. 
Table 13. Thermoelectric-power water withdrawals by cooling type, 2010.

[Values may not sum to totals because of independent rounding. All withdrawal values are in million gallons per day]

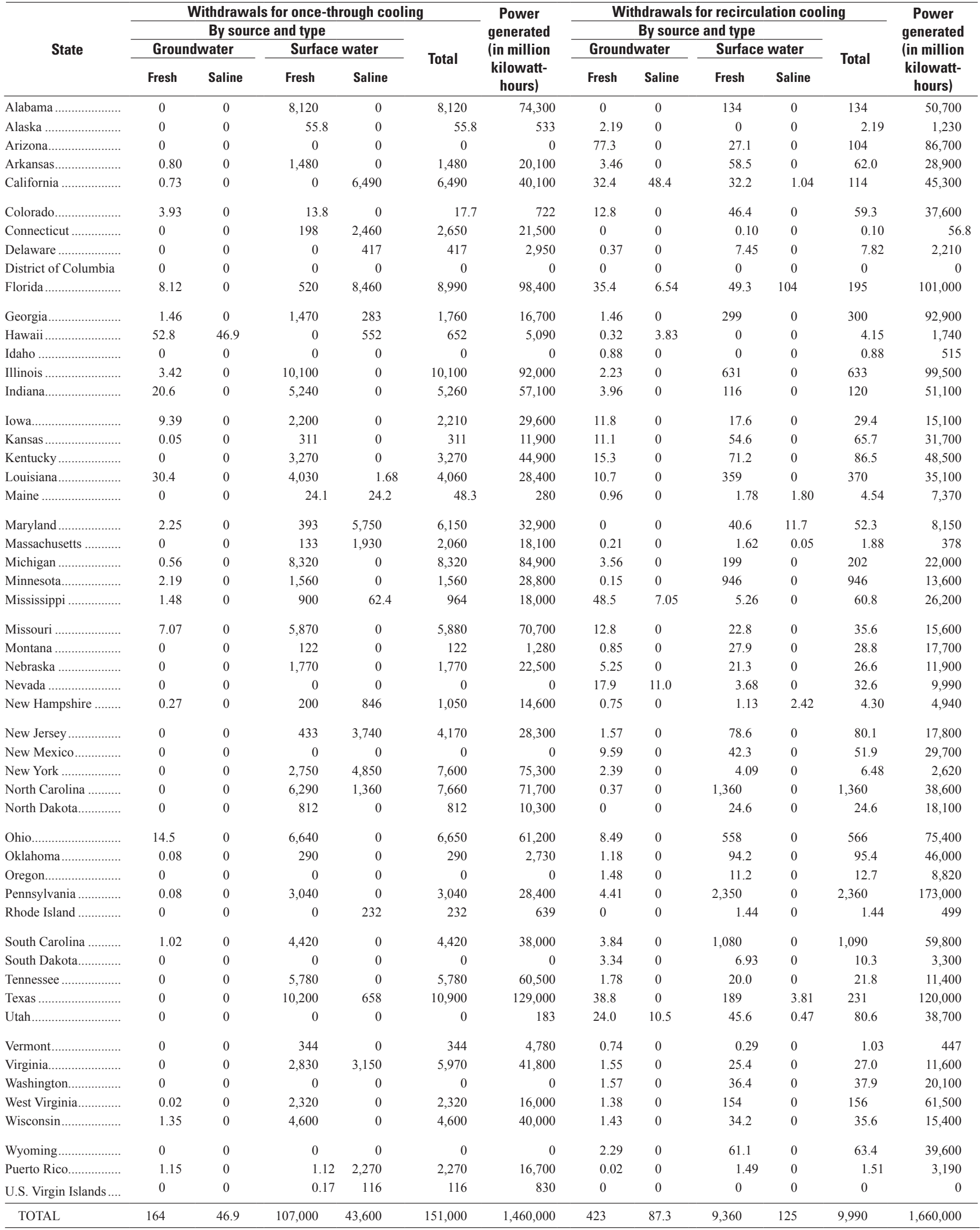




\section{Trends in Water Use, 1950-2010}

The USGS has conducted water-use compilations every 5 years since 1950 (http://water.usgs.gov/watuse/50years.html). A summary of population growth and withdrawal estimates by category of use and source of water is discussed in this section and shown in table 14 for each 5-year period from 1950 through 2010 . These trends are shown graphically for freshwater uses in figure 13 and total uses in figure 14.

Table 14 shows withdrawals for categories of use for each compilation period. Some categories were compiled and presented differently since compilations were begun. For example, self-supplied domestic and livestock withdrawals are shown separately in table 14; however, they were combined as "rural" in the 1950 and 1955 reports. Prior to 1985, the industrial water-use category included withdrawals for commercial, mining, and aquaculture; after 1985 these categories were estimated separately. Water use at fish hatcheries was reported as commercial use in 1990 and 1995, but was included in the aquaculture category for 2000, 2005, and again in 2010 . Estimates of commercial withdrawals were not compiled nationally for 2000, 2005 or 2010. Totals in table 14 represent the most current data and incorporate revisions to previously published data; therefore, percentage differences and national totals may be slightly different from previous reports.

Total withdrawals for all categories of use in 2010 were estimated to be $355 \mathrm{Bgal} / \mathrm{d}$, a level of withdrawal not reported since before 1970. Total withdrawals in 2010 were 13 percent less than in 2005, causing an abrupt downward shift to the mostly steady trend exhibited since 1985 . This downward trend was caused by significant declines in the largest categories of use, including thermoelectric power, irrigation, public supply, and industrial. Categories with larger withdrawals in 2010 than in 2005 were mining and aquaculture, but these categories are small and increased total withdrawals for those categories of use did not offset the much larger overall decrease of $54 \mathrm{Bgal} / \mathrm{d}$ from the other uses.

Although the trend in total population since 1950 has been steadily upward, the rate of increase has varied over time (table 14). Most recently, total population in the United States increased only 4 percent between 2005 and 2010, or an additional 12.3 million people. This continues the upward trend in total population growth exhibited since 1950, but at a slightly slower rate. Historically, decadal growth rates in the United States were at their highest between 1950 and 1960, with an 19 percent increase from an additional 29 million people. Then growth rates exhibited an overall steady trend between 1960 and 1990, with no more than a 27 million person per year increase in the 30 year period. The rates sharply increased with a 13.2 percent increase (32.7 million) between 1990 and 2000 and were most recently at a 9.7 percent (27.3 million) increase from 2000 to 2010. (U.S. Census Bureau, 2011). In the last decade, population growth rate recorded was much faster in Southern and Western States
(14.3 and 13.8 percent, respectively) compared to Midwestern States (3.9 percent) and Northeastern States (3.2 percent).

Thermoelectric power continued to account for the largest withdrawals for any category of use at $161 \mathrm{Bgal} / \mathrm{d}$, or 45 percent of the total withdrawals from all categories of use. Total thermoelectric-power withdrawals in 2010 were about 20 percent less than in 2005; freshwater withdrawals were 18 percent less, and saline-water withdrawals were 24 percent less. These were the largest reductions in total withdrawals between 2005 and 2010 when considering all uses and accounted for the majority of the 13 percent decline in total withdrawals for all uses. Total withdrawals for thermoelectric power in 1985 were 11 percent less than in 1980, and fluctuations in total withdrawals during the 5 -year intervals between 1985 and 2005 were never more than 5 percent.

Several factors may be attributed to the 20 percent decline in total thermoelectric withdrawals. Since the 1970s, an increasing number of powerplants were built with or converted to recirculating cooling systems or dry cooling systems, which use less water than powerplants with oncethrough cooling systems. Withdrawals at powerplants have declined in some States due to the implementation of new rules designed to minimize adverse effects to aquatic life at powerplant intakes. The decrease in use of coal and increase in use of natural gas and new powerplants coming online that use more water-efficient cooling technology also have helped to reduce withdrawals for thermoelectric power.

The plant-specific information pertaining to cooling systems, water sources, and net power generation provided estimates of thermoelectric-power withdrawals from the linked heat and water budget models. These data were used in place of data reported to EIA if plant-reported data were not available. Finally, between 2005 and 2010, the closing of once-through plants also contributed significantly to the reduction in total withdrawals. Withdrawals in California were nearly 50 percent less between 2005 and 2010 primarily due to plant closures and upgrades to intakes and cooling systems implemented in order to comply with State regulations (California State Water Resources Control Board, 2013).

Net power generation associated with thermoelectric power was 3,130,000 gWh in 2010 (table 12), or about 2.5 percent less than the $3,200,000 \mathrm{gWh}$ in 2005 . Net power generation increased nearly 50 percent between 1985 $(2,140,000 \mathrm{gWh})$ and 2010. On average, 19 gallons were used to produce $1 \mathrm{kWh}$ of electricity in 2010, compared to almost $23 \mathrm{gal} / \mathrm{kWh}$ in 2005.

Irrigation withdrawals were 9 percent less between 2005 and 2010 , from $127 \mathrm{Bgal} / \mathrm{d}$ to $115 \mathrm{Bgal} / \mathrm{d}$, a level not reported since before 1965 . This marks the second consecutive 5 -year period of decline in irrigation withdrawals and placed 2010 withdrawals 23 percent less than 1980, when withdrawals peaked. However, irrigation withdrawals remained 
Table 14. Trends in estimated water use in the United States, 1950-2010.

[Data for 2005 and earlier from Kenny and others (2009). Water-use data are in billion gallons per day (thousand million gallons per day) and are rounded to two significant figures for 1950-80, and to three significant figures for 1985-2005; percentage change is calculated from unrounded numbers. Geographic extent: 1950, 48 States and District of Columbia, and Hawaii; 1955, 48 States and District of Columbia; 1960 and 1975-2005, 50 States and District of Columbia, Puerto Rico, and U.S. Virgin Islands; 1965-70, 50 States and District of Columbia, and Puerto Rico]

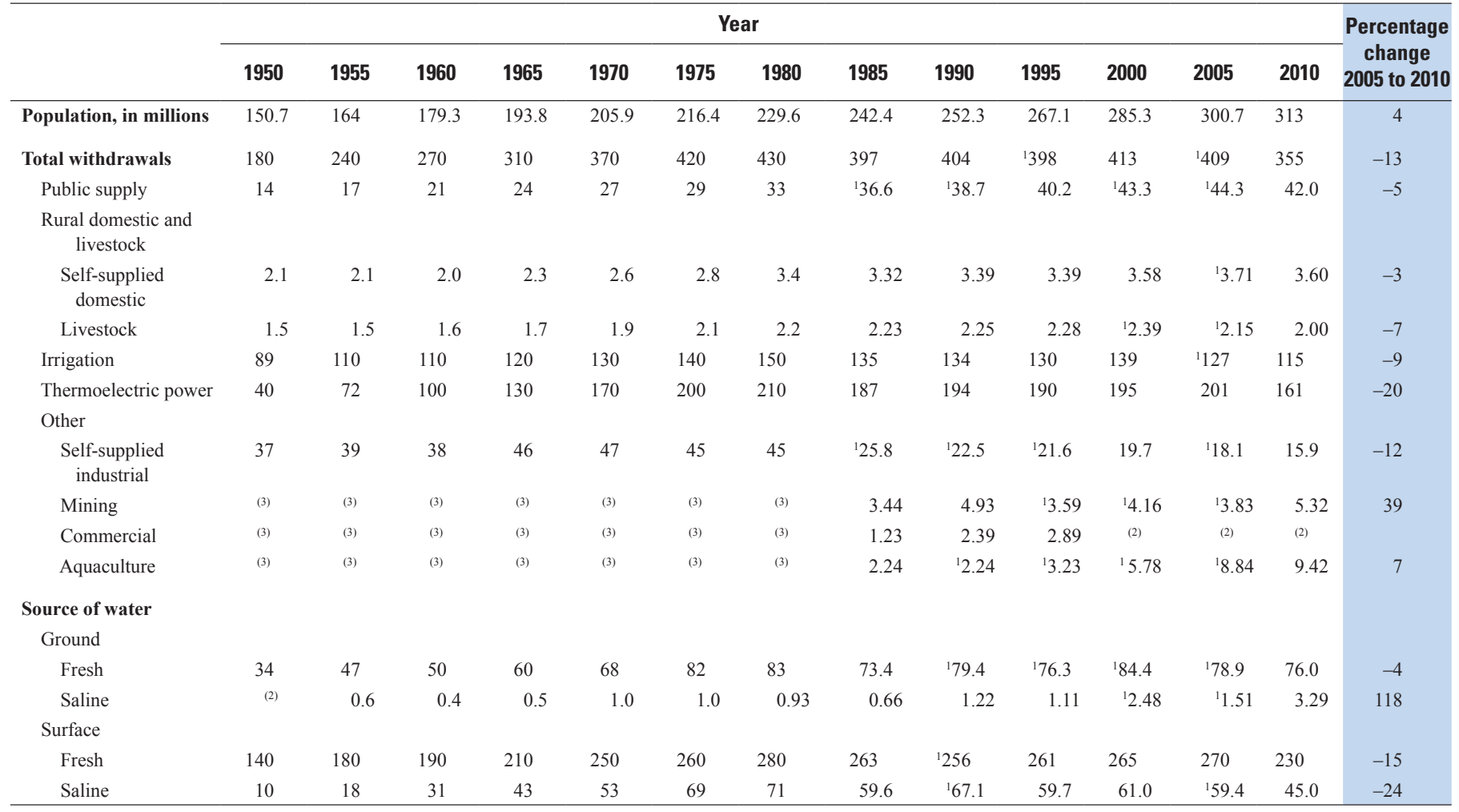

${ }^{1}$ Revised data values.

${ }^{2}$ Data not available.

${ }^{3}$ Included in self-supplied industrial category.

the second-largest category of use after thermoelectric. In 1950, irrigation withdrawals accounted for 64 percent of total freshwater withdrawals excluding thermoelectric, and in 2010 irrigation withdrawals accounted for 61 percent of total freshwater withdrawals excluding thermoelectric. Between 1985 and 2010, the majority of irrigation water was supplied by surface-water sources, ranging from 66 percent in 1985 to 57 percent in 2010. The use of more water-efficient irrigation systems continued to increase with nearly 3 percent more irrigated acres using sprinkler systems in 2010 than in 2005 . Nearly 2 percent fewer irrigated acres were reported using flood (surface) irrigation systems in 2010 compared to 2005. Microirrigation systems showed the largest percentage increase between 2005 and 2010, with 14 percent more irrigated acres with this type of system. Total irrigated acres were only 2 percent more in 2010 than in 2005.

Public-supply withdrawals in 2010 were 5 percent less than in 2005 , decreasing from $44.3 \mathrm{Bgal} / \mathrm{d}$ to $42.0 \mathrm{Bgal} / \mathrm{d}$ and marking the first decline since public-supply withdrawals were initially reported in 1950. Total public-supply withdrawals in 2010 were at levels not reported since prior to 2000. During decadal periods between 1950 and 1960, public-supply withdrawals increased 50 percent in conjunction with the high population growth rates during those periods. Percentage increases in public-supply withdrawals during the next three decadal periods between 1960 and 1990 averaged 23 percent, again coinciding with the rate of growth in population during the same time periods. Between 1990 and 2000, the rate of increase in public-supply withdrawals was lower at 12 percent. Between 1990 and 2010, public-supply withdrawals have been roughly 60 percent from surface water and 40 percent from groundwater sources. The percentage of the population that is served from public-supply withdrawals has increased from 62 percent in 1950 to 86 percent in 2010.

Self-supplied domestic withdrawals declined 3 percent between 2005 and 2010, from 3.71 Bgal/d to $3.60 \mathrm{Bgal} / \mathrm{d}$. Since 1985, the rate of change in self-supplied domestic withdrawals has remained fairly steady with, at most, a 6 percent increase (1995-2000). Between 1985 and 2010, the percentage of total population that was self-supplied has continuously declined, from about 18 percent to 14 percent. The average per capita use for self-supplied domestic withdrawals decreased from 89 gallons per day in 2005 to 81 gallons per day in 2010. Estimates of self-supplied domestic withdrawals are computed either using a standard coefficient of use or a coefficient derived from data about public-supply domestic 


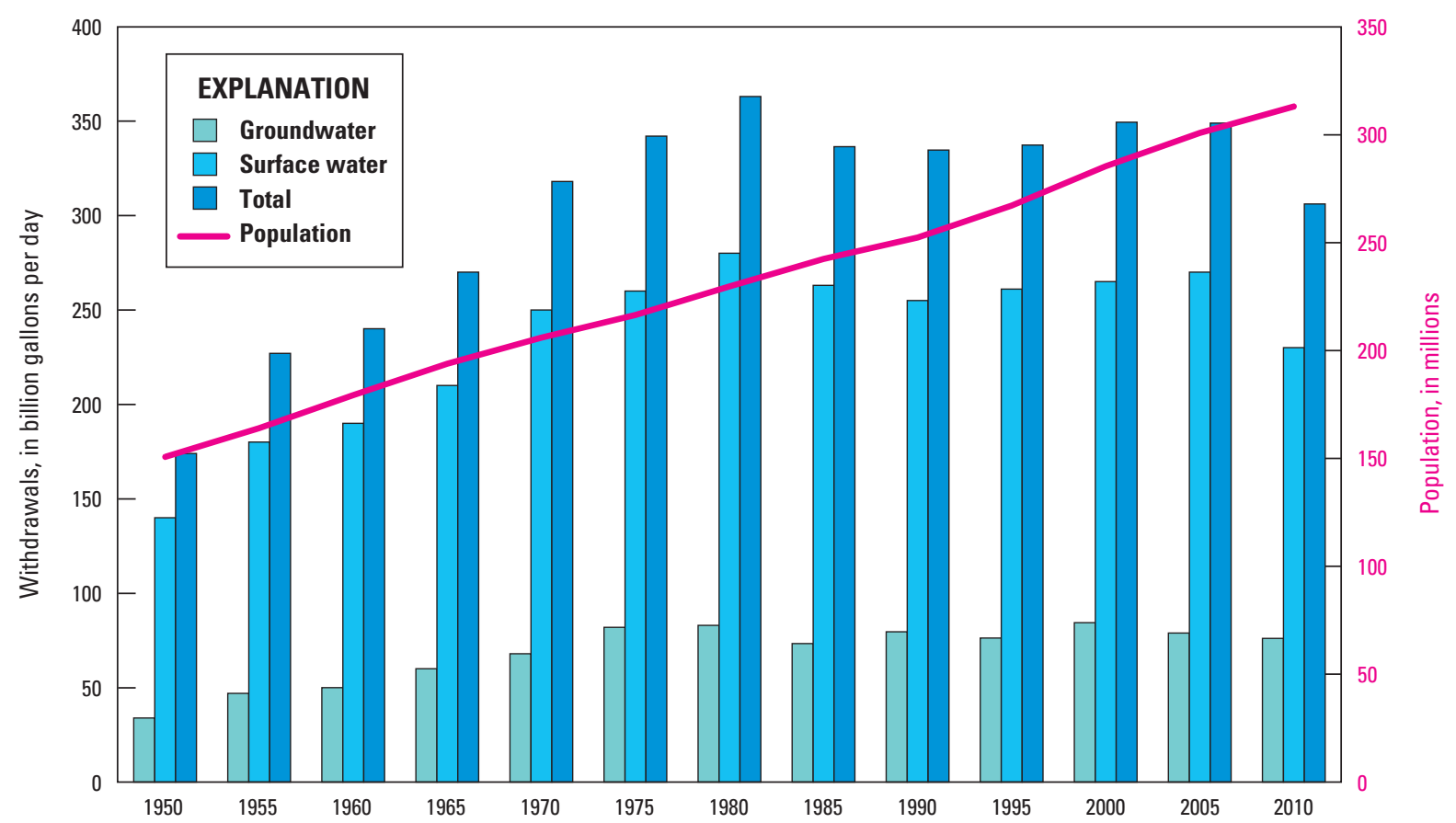

Figure 13. Trends in population and freshwater withdrawals by source, 1950-2010.

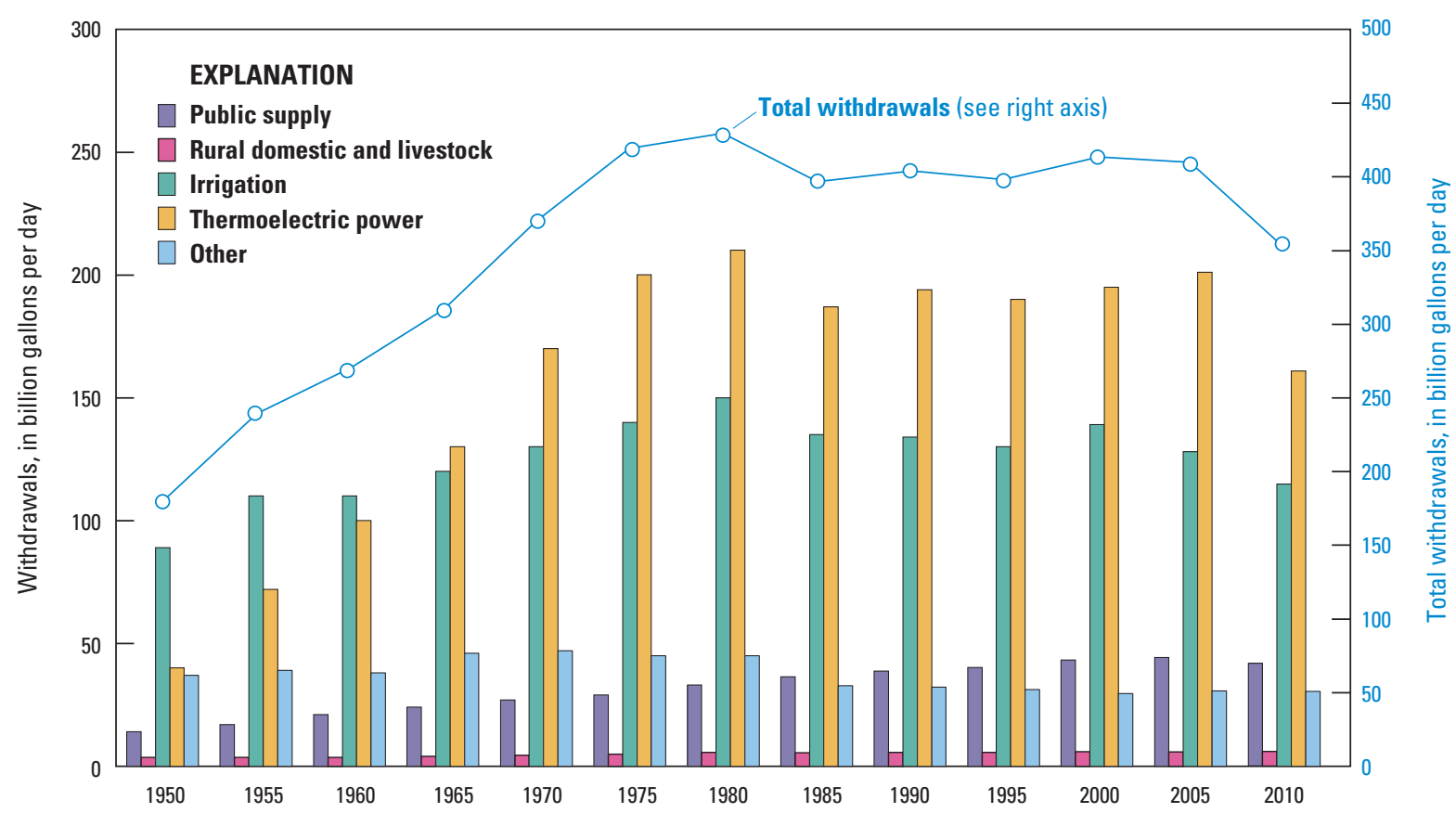

Figure 14. Trends in total water withdrawals by water-use category, 1950-2010. 
deliveries. The national average public-supply domestic delivery per capita use declined from 105 gallons per day in 1985 to 89 gallons per day in 2010 . The decline in the selfsupplied domestic per capita use is most likely a function of the decline in the public-supply domestic delivery per capita use. In particular, California ( 7 percent self-supplied population) reported 179 gpcd for self-supplied domestic use in 2005, and 69 gpcd in 2010. Similarly, Texas (10 percent selfsupplied population) and Wisconsin (30 percent self-supplied population) both reported 8 percent declines in self-supplied domestic per capita use between 2005 and 2010.

Changes in the industrial category can be compared for 1985 through 2010, which are the years this category was compiled separately for commercial, mining, and aquaculture uses. Total industrial withdrawals decreased 12 percent between 2005 and 2010, continuing the decline shown each period since 1985. Total industrial withdrawals decreased by 38 percent between 1985 to 2010 , from $25.8 \mathrm{Bgal} / \mathrm{d}$ in 1985 to $15.9 \mathrm{Bgal} / \mathrm{d}$ in 2010 . Groundwater provided 14 percent of the total industrial withdrawals in 1985; this proportion has been in the range of 17 to 18 percent since. Almost all of the industrial groundwater withdrawals were freshwater. Fresh surfacewater withdrawals have accounted for more than 90 percent of surface-water withdrawals for industrial use since 1995 and was 93 percent in 2010.

Declines in industrial withdrawals reflects greater efficiencies in industrial processes and an emphasis on water reuse and recycling within industrial facilities, both driven by environmental regulations and limited availability of freshwater resources in some areas. The larger decline in industrial withdrawals from 2005 to 2010 compared to 2000 to 2005 (12 percent compared to 8 percent) may be due in part to lower industrial production in the major water-using industries of wood products, primary metals, paper, and chemicals, all of which had lower production following the 2008 U.S. recession (Board of Governors of the Federal Reserve System, 2014).

Livestock, mining, and aquaculture withdrawals were included with other categories prior to 1985 . Livestock initially was included with rural domestic, but since 1960 has been estimated as a separate category and has consistently accounted for about 1 percent of total withdrawals excluding thermoelectric throughout the 1960-2010 period. Withdrawals for livestock decreased 7 percent from $2.15 \mathrm{Bgal} / \mathrm{d}$ in 2005 to $2.00 \mathrm{Bgal} / \mathrm{d}$ in 2010 , showing the second consecutive period of decline. Livestock withdrawals in 2010 were 16 percent less than the peak year of 2000 , when $2.39 \mathrm{Bgal} / \mathrm{d}$ was reported.

Mining withdrawals were $5.32 \mathrm{Bgal} / \mathrm{d}$ in 2010 , or a 39 percent increase over 2005 (3.83 Bgal/d). This represented the largest percentage increase of any category of use between 2005 and 2010, but since mining is a relatively small category in terms of total withdrawals, the increase did not offset the large national decreases in total water use. Prior to 1985 , mining was included in other industrial withdrawals, but since 1985 has represented from 1.6 to 2.7 percent of total withdrawals excluding thermoelectric. Trends in mining withdrawals have fluctuated between 1985 and 2010, ranging from an increase of 43 percent between 1985 and 1990, followed by a 27 percent decrease between 1990 and 1995 .

Aquaculture withdrawals were $9.42 \mathrm{Bgal} / \mathrm{d}$ in 2010, or a 7 percent increase from 2005. Aquaculture was the other category along with mining that showed an increase in withdrawals between 2005 and 2010. Since 1985, aquaculture has grown from 1 percent of total withdrawals excluding thermoelectric to almost 5 percent in 2010 with the most increase change between 1995 and 2000 when the total withdrawals increased nearly 80 percent.

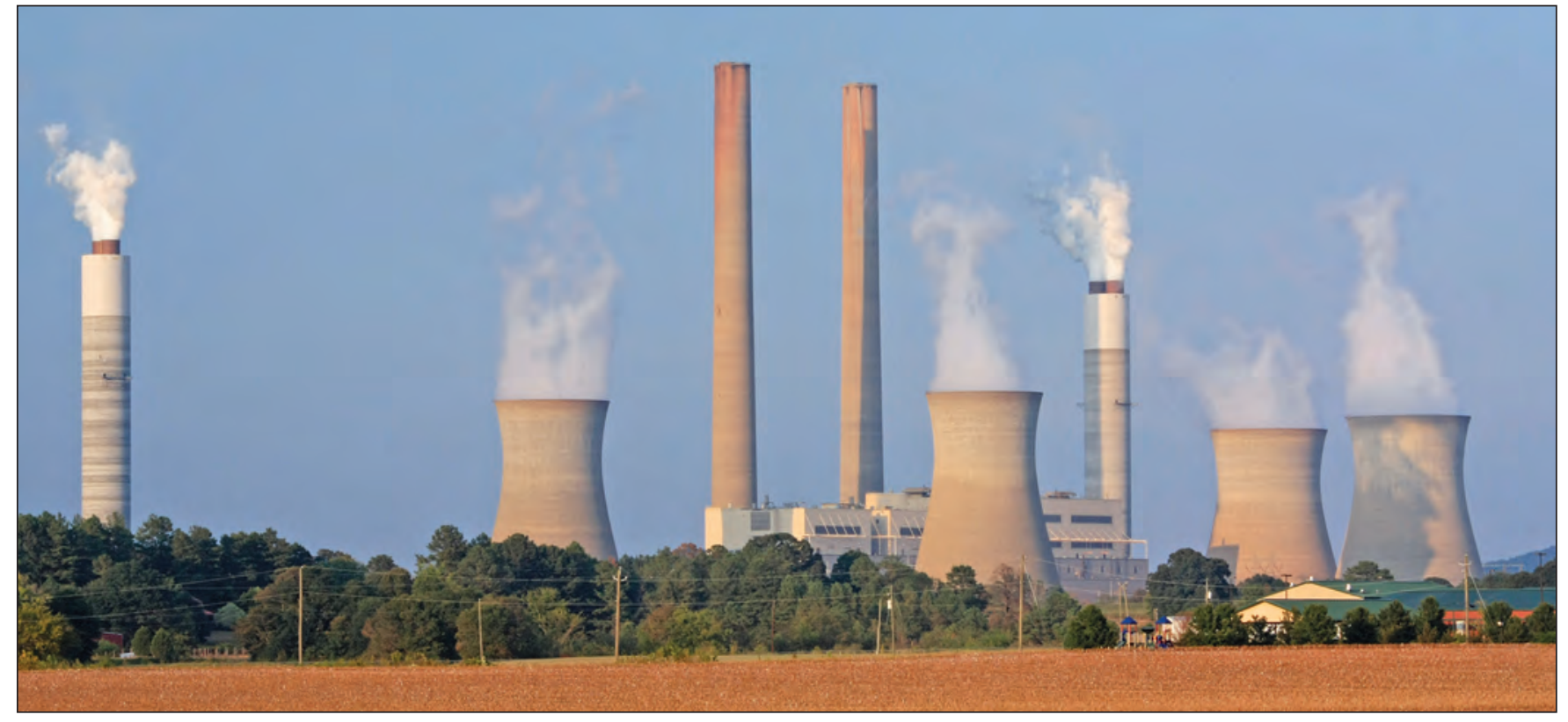

The Plant Bowen coal-fired powerplant outside Euharlee in Bartow County, Georgia. Photo by Alan Cressler, USGS. 


\section{References Cited}

Board of Governors of the Federal Reserve System, 2014, Industrial Production and Capacity Utilization-G.17, accessed September 19, 2014, at http://www.federalreserve. gov/releases/g17/About.htm.

California State Water Resources Control Board, 2013, Once-through cooling policy protects marine life and insures electric grid reliability, accessed July 7, 2014, at http://www.waterboards.ca.gov/publications_forms/ publications/factsheets/docs/oncethroughcooling.pdf.

Dickens, J.M., Forbes, B.T., Cobean, D.S., and Tadayon, Saeid, 2011, Documentation of methods and inventory of irrigation data collected for the 2000 and 2005 U.S. Geological Survey "Estimated use of water in the United States," comparison of USGS-compiled irrigation data to other sources, and recommendations for future compilations: U.S. Geological Survey Scientific Investigations Report 2011-5166, 60 p., http://pubs.usgs.gov/sir/2011/5166/.

Diehl, T.H., and Harris, M.A., 2014, Withdrawals and consumption of water by thermoelectric power plants in the United States, 2010: U.S. Geological Survey Scientific Investigations Report 2014-5184, 28 p., http://dx.doi.RU $\square$ 10.3133/sir20145184.

Diehl, T.H., Harris, M.A., Murphy, J.C., Hutson, S.S., and Ladd, D.E., 2013, Methods for estimating water consumption for thermoelectric power plants in the United States: U.S. Geological Survey Scientific Investigations Report 2013-5188, 78 p., http://dx.doi.org/10.3133/sir20135188.

Hutson, S.S., compiler, 2007, Guidelines for preparation of State water-use estimates for 2005: U.S. Geological Survey Techniques and Methods, book 4, chap. E1, 28 p. (Also available at http://pubs.usgs.gov/tm/2007/tm4e1.)

Hutson, S.S., Barber, N.L., Kenny, J.F., Linsey, K.S., Lumia, D.S., and Maupin, M.A., 2004, Estimated use of water in the United States in 2000: U.S. Geological Survey Circular 1268, 46 p., http://pubs.usgs.gov/circ/2004/circ1268/.

Kenny, J.F., Barber, N.L., Hutson, S.S., Linsey, K.S., Lovelace, J.K., and Maupin, M.A., 2009, Estimated use of water in the United States in 2005: U.S. Geological Survey Circular 1344, 52 p., http://pubs.usgs.gov/circ/1344/.

Levin, S.B., and Zarriello, P.J., 2013, Estimating irrigation water use in the humid eastern United States: U.S. Geological Survey Scientific Investigations Report 2013-5066, 32 p., http://pubs.usgs.gov/sir/2013/5066/.

Lovelace, J.K., 2009a, Method for estimating water withdrawals for livestock in the United States, 2005: U.S. Geological Survey Scientific Investigations Report 2009-5041, 7 p., http://pubs.usgs.gov/sir/2009/5041/.

Lovelace, J.K., 2009b, Methods for estimating water withdrawals for mining in the United States, 2005: U.S. Geological Survey Scientific Investigations Report 2009-5053, 7 p., http://pubs.usgs.gov/sir/2009/5053/.
MacKichan, K.A., 1951, Estimated use of water in the United States, 1950: U.S. Geological Survey Circular 115, 13 p.

MacKichan, K.A., 1957, Estimated use of water in the United States, 1955: U.S. Geological Survey Circular 398, 18 p.

MacKichan, K.A., and Kammerer, J.C., 1961, Estimated use of water in the United States, 1960: U.S. Geological Survey Circular 456, $26 \mathrm{p}$.

Murray, C.R., 1968, Estimated use of water in the United States, 1965: U.S. Geological Survey Circular 556, 53 p.

Murray, C.R., and Reeves, E.B., 1972, Estimated use of water in the United States, 1970: U.S. Geological Survey Circular 676, $37 \mathrm{p}$.

Murray, C.R., and Reeves, E.B., 1977, Estimated use of water in the United States in 1975: U.S. Geological Survey Circular 765, $39 \mathrm{p}$.

National Oceanic and Atmospheric Administration, National Climate Data Center, 2010, State of the climate, Entire report-annual 2010, accessed August 25, 2014, at http://www.ncdc.noaa.gov/sotc/2010/13.

Price, C,V., and Maupin, M,A., 2014, Documentation for the U.S. Geological Survey Public Supply Database (PSDB) - A database of permitted public-supply wells, surface-water intakes, and systems in the United States: U.S. Geological Survey Open-File Report 2014-1212, 14 p.

Solley, W.B., Chase, E.B., and Mann, W.B., IV, 1983, Estimated use of water in the United States in 1980: U.S. Geological Survey Circular 1001, 56 p.

Solley, W.B., Merk, C.F., and Pierce, R.R., 1988, Estimated use of water in the United States in 1985: U.S. Geological Survey Circular 1004, 82 p.

Solley, W.B., Pierce, R.R., and Perlman, H.A., 1993, Estimated use of water in the United States in 1990: U.S. Geological Survey Circular 1081, 76 p.

Solley, W.B., Pierce, R.R., and Perlman, H.A., 1998, Estimated use of water in the United States in 1995: U.S. Geological Survey Circular 1200, 71 p.

U.S. Census Bureau, 2011, Population distribution and changes - 2000 to 2010: United States Census Bureau, access July 7, 2014, at http://www.census.gov/prod/ cen2010/briefs/c2010br-01.pdf.

U.S. Department of Energy, 2011, Annual energy outlook 2011 with projections to 2035: U.S. Energy Information Administration, Office of Integrated and International Energy Analysis, DOE/EIA-0383(2011), 235 p.

U.S. Environmental Protection Agency, 2014, Laws \& Statues-The Safe Drinking Water Act, accessed March 14, 2014, at http://waterepa.gov/lawsregs/ guidance/sdwa/laws_statutes.cfm.

Veil, J.A., 2007, Use of reclaimed water for power plant cooling: Argonne, Ill., Argonne National Laboratory, Environmental Science Division, ANL/EVS/R-07/3, 60 p. 


\section{Glossary}

The following terms are referenced in the text or are part of the water-use Circular series.

animal-specialties water use Water use associated with the production of fish in captivity, except for fish hatcheries, and the raising of horses and such fur-bearing animals as rabbits and pets. Animal-specialties water-use estimates were included in the 1990 and 1995 water-use Circulars, but were combined with the livestock categories or aquaculture categories beginning in 2000. See also aquaculture water use, fish-farm water use, livestock water use, and rural water use.

aquaculture water use Water use associated with the farming of organisms that live in water (such as finfish and shellfish) and offstream water use associated with fish hatcheries. See also fishfarm water use, fish-hatchery water use, animalspecialties water use, and livestock water use.

closed-loop cooling system See recirculation cooling system.

commercial water use Water for motels, hotels, restaurants, office buildings, other commercial facilities, military and nonmilitary institutions, and (for 1990 and 1995) offstream fish hatcheries. Water may be obtained from a public-supply system or may be self-supplied. Commercial water-use estimates were included in some previous water-use Circulars but were omitted beginning in 2000. See also fish-hatchery water use, public-supply water use, public-supply deliveries, and self-supplied water use.

consumptive use The part of water withdrawn that is evaporated, transpired, incorporated into products or crops, consumed by humans or livestock, or otherwise removed from the immediate water environment. Consumptive-use estimates were included in some previous wateruse Circulars but were omitted beginning in 2000 . Also referred to as water consumed.

conveyance loss Water that is lost in transit from a pipe, canal, conduit, or ditch by leakage or evaporation. Generally, the water is not available for further use; however, leakage from an irrigation ditch, for example, may percolate to a groundwater source and be available for further use. Conveyance-loss estimates were included in some previous water-use Circulars but were omitted beginning in 2000. See also irrigation water use. cooling system An equipment system that provides water for cooling purposes, such as to condensers at powerplants or at factories. May include water intakes, outlets, cooling towers, ponds, canals, pumps, and pipes. See also coolingsystem type, industrial water use, and thermoelectric-power water use.

cooling-system type Defined as either oncethrough or recirculation cooling system. See also industrial water use, once-through cooling system, recirculation cooling system, and thermoelectricpower water use.

domestic water use Water used for indoor household purposes such as drinking, food preparation, bathing, washing clothes and dishes, flushing toilets, and outdoor purposes such as watering lawns and gardens. Domestic water use includes water provided to households by a public water supply (domestic deliveries from public suppliers) and self-supplied water. See also publicsupply deliveries, public-supply water use, rural water use, and self-supplied water use.

fish-farm water use Water used for the production of finfish and shellfish under controlled feeding, sanitation, and harvesting procedures for commercial purposes. Water use by fish farms is classified in the aquaculture category. See also animal-specialties water use, aquaculture water use, and fish-hatchery water use.

fish-hatchery water use Water used for raising fish for later release and in association with the operation of fish hatcheries or fishing preserves. Fish-hatchery water use has been included in the aquaculture category since 2000. See also aquaculture water use, commercial water use, and fish-farm water use.

freshwater Water that contains less than 1,000 milligrams per liter $(\mathrm{mg} / \mathrm{L})$ of dissolved solids. Generally, water with more than $500 \mathrm{mg} / \mathrm{L}$ of dissolved solids is undesirable for drinking and many industrial uses. See also saline water.

industrial water use Water used for fabrication, processing, washing, and cooling. Includes industries such as chemical and allied products, food, mining, paper and allied products, petroleum refining, and steel. Term used in previous 
water-use Circulars to describe the combined public-supply deliveries to industrial users and self-supplied industrial withdrawals. Since 2000, industrial water use refers only to self-supplied industrial withdrawals. See also cooling system, cooling-system type, mining water use, publicsupply deliveries, public-supply water use, and self-supplied water use.

instream use Water that is used, but not withdrawn, from a surface-water source for such purposes as hydroelectric-power generation, navigation, water-quality improvement, fish propagation, and recreation. Instream water-use estimates for hydroelectric power were included in some previous water-use Circulars but were omitted since 2000 .

irrigation district A cooperative, self-governing public corporation set up as a subdivision of the State government, with definite geographic boundaries, organized, and having taxing power to obtain and distribute water for irrigation of lands within the district. Created under the authority of a State legislature with the consent of a designated fraction of the landowners or citizens. See also irrigation water use.

irrigation water use Water that is applied by an irrigation system to assist crop and pasture growth, or to maintain vegetation on recreational lands such as parks and golf courses. Irrigation includes water that is applied for pre-irrigation, frost protection, chemical application, weed control, field preparation, crop cooling, harvesting, dust suppression, leaching of salts from the root zone, and conveyance losses. See also conveyance loss, microirrigation system, sprinkler irrigation system, and surface irrigation system.

livestock water use Water used for livestock watering, feedlots, dairy operations, and other on-farm needs. Types of livestock include dairy cows and heifers, beef cattle and calves, sheep and lambs, goats, hogs and pigs, horses and poultry. See also animal-specialties water use, aquaculture water use, and rural water use.

microirrigation system An irrigation system that wets only a discrete portion of the soil surface in the vicinity of the plant by means of applicators (such as orifices, emitters, porous tubing, or perforated pipe) and operated under low pressure. The applicators may be placed on or below the surface of the ground or suspended from supports. See also irrigation water use, sprinkler irrigation system, and surface irrigation system. mining water use Water used for the extraction of naturally occurring minerals including solids (such as coal, sand, gravel, and other ores), liquids (such as crude petroleum), and gases (such as natural gas). Also includes uses associated with quarrying, milling of mined materials, injection of water for secondary oil recovery or for unconventional oil and gas recovery (such as hydraulic fracturing), and other operations associated with mining activity. Does not include water associated with dewatering of the aquifer that is not put to beneficial use. Also does not include water used in processing, such as smelting, refining petroleum, or slurry pipeline operations. These processing uses are included in industrial water use. See also industrial water use and self-supplied water use.

offstream use Water withdrawn or diverted from a groundwater or surface-water source for aquaculture, commercial, self-supplied domestic, industrial, irrigation, livestock, mining, public supply, thermoelectric power, and other uses. See also entries for each of these categories of use.

once-through cooling system Also known as open-loop cooling system. Cooling system in which the water is withdrawn from a source, circulated through the heat exchangers, and then returned to a body of water at a higher temperature. See also cooling system, cooling-system type, industrial water use, and thermoelectricpower water use.

public-supply deliveries Amount of water delivered from a public supplier to users for domestic, commercial, industrial, thermoelectric-power, or public-use purposes. Estimates of deliveries for each purpose were provided for 1995 and earlier years, but not for 2000. For 2005 and 2010, only domestic deliveries were estimated nationally. See also commercial water use, domestic water use, industrial water use, public-supply water use, public water use, and thermoelectric-power use.

public-supply water use Water withdrawn by public and private water suppliers that furnish water to at least 25 people or have a minimum of 15 connections. Public suppliers provide water for a variety of uses, such as domestic, commercial, industrial, thermoelectric-power, and public water use. See also commercial water use, domestic water use, industrial water use, public-supply deliveries, public water use, and thermoelectricpower water use. 
public water use Water supplied from a public supplier and used for such purposes as firefighting, street washing, flushing of water lines, and maintaining municipal parks and swimming pools. Generally, public-use water is not billed by the public supplier. See also public-supply deliveries and public-supply water use.

recirculation cooling system Also known as closed-loop cooling system. Water is withdrawn from a source, circulated through heat exchangers, cooled, and then re-used in the same process. Recirculation cooling systems may use induced draft cooling towers, forced draft cooling towers, cooling ponds, or canals. See also cooling system, cooling-system type, industrial water use, and thermoelectric-power water use.

reclaimed wastewater Wastewater-treatment plant effluent that has been diverted for beneficial uses such as irrigation, industry, or thermoelectricpower cooling instead of being released to a natural waterway or aquifer. See also water use.

return flow Water that reaches a groundwater or surface-water source after release from the point of use and thus becomes available for further use. See also water use.

rural water use Water used in suburban or farm areas for domestic and livestock needs. The water generally is self-supplied, and includes domestic use, drinking water for livestock, and other uses such as dairy sanitation, cleaning, and waste disposal. Term used in previous water-use Circulars. See also animal-specialties water use, domestic water use, livestock water use, and selfsupplied water use.

saline water Water that contains $1,000 \mathrm{mg} / \mathrm{L}$ or more of dissolved solids. See also freshwater.

self-supplied water use Water withdrawn from a groundwater or surface-water source by a user rather than being obtained from a public-supply source.

sprinkler irrigation system An irrigation system in which water is applied by means of perforated pipes or nozzles operated under pressure so as to form a spray pattern. See also irrigation water use, microirrigation system, and surface irrigation system.

standard industrial classification (SIC) codes Four-digit codes established by the Office of Management and Budget, published in 1987, and used in the classification of establishments by type of activity in which they are engaged. surface irrigation system Irrigation by means of flood, furrow, or gravity. Flood irrigation is the application of irrigation water in which the entire soil surface is covered by ponded water. Furrow is a partial surface-flooding method of irrigation normally used with clean-tilled crops in which water is applied in furrows or rows of sufficient capacity to contain the design irrigation stream. Gravity is an irrigation method in which water is not pumped, but flows in ditches or pipes and is distributed by gravity. See also irrigation water use, microirrigation system, and sprinkler irrigation system.

thermoelectric-power water use Water used in the process of generating electricity with steamdriven turbine generators. Term used in previous water-use Circulars to describe the combined public-supply deliveries to thermoelectric-powerplants and self-supplied thermoelectric-power withdrawals. Since 2000, thermoelectric-power water use refers only to self-supplied thermoelectric-power withdrawals. See also cooling system, cooling-system type, public-supply water use, and self-supplied water use.

wastewater-treatment return flow Term used in previous water-use Circulars to describe water returned to the hydrologic system by wastewatertreatment facilities. See also water use.

water use In a restrictive sense, the term refers to water that is withdrawn for a specific purpose, such as for public supply, domestic use, irrigation, thermoelectric-power cooling, or industrial processing. In previous water-use Circulars, water use for the domestic, commercial, industrial, and thermoelectric categories included both selfsupplied withdrawals and deliveries from public supply. More broadly, water use pertains to the interaction of humans with and influence on the hydrologic cycle, and includes elements such as water withdrawal, delivery, consumptive use, wastewater release, reclaimed wastewater, return flow, and instream use. See also offstream use and instream use.

water withdrawal Water removed from a groundwater or surface-water source for use. See also offstream use and self-supplied water use.

watt-hour (Wh) An electrical energy unit of measure equal to 1 watt of power supplied to, or taken from, an electric circuit steadily for 1 hour. 


\section{Cooperating Agencies and Organizations}

The following State, regional, and local organizations provided assistance and data as part of the water-use compilation. In addition, State, regional, and national offices of the U.S. Army Corps of Engineers, U.S. Bureau of Reclamation, U.S. Department of Commerce, U.S. Department of Energy, U.S. Environmental Protection Agency, U.S. Fish and Wildlife Service, National Agricultural Statistics Service, National Oceanic and Atmospheric Administration, National Weather Service, Natural Resources Conservation Service, and other Federal agencies provided assistance and data for various States.

\section{Alabama}

Alabama Department of Economic and Community Development, Office of Water Resources, Water Management Branch

Alabama Department of Environmental Management, Drinking Water Branch

\section{Alaska}

Alaska Department of Administration, Oil and Gas Conservation Commission

Alaska Department of Commerce, Community and Economic Development

Alaska Department of Environmental Conservation, Division of Water

Alaska Department of Fish and Game

Alaska Department of Natural Resources, Division of Agriculture

Alaska Department of Natural Resources, Division of Geological and Geophysical Surveys

Alaska Department of Natural Resources, Division of Mining Land and Water

Alaska Department of Natural Resources, Division of Oil and Gas

University of Alaska, Cooperative Extension Service

\section{Arizona}

Arizona Department of Water Resources

\section{Arkansas}

Arkansas Association of Conservation Districts

Arkansas Department of Health, Engineering Division

Arkansas Natural Resources Commission

\section{California}

California Department of Food and Agriculture, Annual County Agriculture Commissioner Reports

California Department of Public Health

California Department of Water Resources, Land and Water Use

California State Water Resources Control Board, Water Recycling Funding Program

Food and Agricultural Organization of the United Nations

Golf Course Superintendents Association of America

\section{Colorado}

Colorado Department of Health and Environment

Colorado Division of Local Affairs

Colorado Division of Reclamation Mining and Safety

Colorado Division of Water Resources

Colorado Oil and Gas Conservation Commission

Colorado Water Conservation Board

Rocky Mountain Golf Course Superintendents Association

\section{Connecticut}

State of Connecticut Department of Environmental Protection

\section{Delaware}

Calpine Mid-Atlantic Generation LLC

City of Lewes, Delaware

City of Newark, Delaware

City of Wilmington, Brandywine Pumping Station, Delaware

Cogentrix (Logan Generating Co. LP)

Delaware Agricultural Extension Service

Delaware City Refinery (former Premcor)

Delaware Department of Natural Resources and Environmental Control

FPL Energy, Marcus Hook, LP

General Chemical Corp.

Indian River Power, LLC

NACP irrigated data (2012)

NRG Indian River Power LLC

United Water Comp., Delaware

\section{Florida}

Florida Department of Environmental Protection, Office of Water Policy

Northwest Florida Water Management District

South Florida Water Management District

Southwest Florida Water Management District

St. Johns River Water Management District

Suwannee River Water Management District 


\section{Georgia}

Georgia Environmental Protection Division,

Watershed Protection Branch

Georgia Power Company

Georgia Soil and Water Conservation Commission

University of Georgia, Cooperative Extension Service

\section{Hawaii}

Hawaii Department of Water Supply

Hawaiian Electric Company

Honolulu Board of Water Supply

Kauai Department of Water

Maui Department of Water Supply

\section{Idaho}

Idaho Department of Agriculture, Agricultural Statistics Service Idaho Department of Environmental Quality

Idaho Department of Fish and Game

Idaho Department of Labor

Idaho Department of Water Resources

Idaho Power Company

United Water Idaho

University of Idaho, Research and Extension Center at Kimberly

\section{Illinois}

Exelon Corporation

Illinois Association of Wastewater Agencies

Illinois State Water Survey-Illinois Water Inventory Program

U.S. Department of Agriculture, Farm Services Agency

\section{Indiana}

Indiana Department of Natural Resources, Division of Water

\section{Iowa}

Iowa Department of Natural ResourcesWater Allocation and Use Program

\section{Kansas}

Kansas Department of Agriculture-Division of Water Resources Kansas Water Office

\section{Kentucky}

Kentucky Energy and Environment Cabinet, Department of Environmental Protection, Division of Water

\section{Louisiana}

Capitol Area Ground Water Conservation Committee

Louisiana Cooperative Extension Service
Louisiana Department of Environmental Quality

Louisiana Department of Health and Hospitals

Louisiana Department of Transportation and Development

Louisiana Office of Conservation - Injection and Mining Division

Louisiana State University Agricultural Center

Maine

Maine Department of Agriculture

Maine Department of Environmental Protection

Maine Department of Health and Human Services

Maine Geological Survey

Maine Public Utilities Commission

Maryland

Maryland Department of the Environment

\section{Massachusetts}

Massachusetts Department of Environmental Protection

Massachusetts Water Resources Authority

\section{Michigan}

Michigan Department of Environmental Quality, Water Use Program

\section{Minnesota}

Minnesota Department of Natural Resources

\section{Mississippi}

Mississippi Agriculture and Forestry Extension Service

Mississippi Department of Environmental Quality, Office of Land and Water Resources

Mississippi State Department of Health

Yazoo Mississippi Delta Joint Management District

\section{Missouri}

Missouri Department of Natural Resources

\section{Montana}

Dry Prairie Rural Water Authority

Fort Peck Rural County Water District

Hill County Water District

Montana Department of Environmental Quality

Montana Department of Natural Resources and Conservation

Montana Department of Revenue

Montana Fish, Wildlife and Parks

Montana State University, Central Agricultural Research Center

Montana Water Company

Multiple cities in Montana 
PPL Montana

Prairie County Community Hospital

Rosebud Operating Services, Inc.

Valley Capitol, Inc.

\section{Nebraska}

Central Nebraska Public Power and Irrigation District

Central Platte Natural Resource District

Lewis and Clark Natural Resource District

Little Blue Natural Resource District

Lower Big Blue Natural Resource District

Lower Elkhorn Natural Resource District

Lower Loup Natural Resource District

Lower Niobrara Natural Resource District

Lower Platte North Natural Resource District

Lower Republican Natural Resource District

Middle Republican Natural Resource District

Nebraska Association of Natural Resource Districts

Nebraska Department of Natural Resources

Nemaha Natural Resource District

North Platte Natural Resource District

South Platte Natural Resource District

Tri-Basin Natural Resource District

Twin Platte Natural Resource District

Upper Big Blue Natural Resource District

Upper Elkhorn Natural Resource District

Upper Loup Natural Resource District

Upper Niobrara-White Natural Resource District

Upper Republican Natural Resource District

\section{Nevada}

Boulder City Water Department

Carson City Public Works

Chevron Global Power Company

Colorado River Commission

Federal Water Master

Nevada Bureau of Mines and Geology

Nevada Commission on Mineral Resources

Nevada Department of Business and Industry

Nevada Department of Conservation and Natural Resources, Division of Environmental Protection

Nevada Department of Wildlife

Nevada Division of Water Resources
Pershing County Water Conservation District

Southern Nevada Water Authority

Truckee Carson Irrigation District

Truckee Meadows Water Authority

U.S. Nuclear Regulatory Commission

Virgin Valley Water District

\section{New Hampshire}

New Hampshire Department of Environmental Services, Water Management Bureau

\section{New Jersey}

New Jersey Department of Environmental Protection

\section{New Mexico}

New Mexico Office of the State EngineerWater Conservation Bureau

\section{New York}

New York City Department of Environmental Protection

New York State Department of Environmental Conservation

New York State Department of Health

\section{North Carolina}

Carolinas Golf Course Superintendents Association

Duke Energy Company

North Carolina Department of Agriculture and Consumer Services, Agribusiness and Aquaculture

North Carolina Department of Environment and Natural Resources, Division of Water Resources

North Carolina State University, College of Agriculture and Life Sciences, Cooperative Extension

North Carolina State University, College of Agriculture and Life Sciences, Department of Crop Science

Progress Energy Company

\section{North Dakota}

North Dakota Industrial Commission - Oil and Gas Division

North Dakota Regional Water Systems

North Dakota Rural Water Systems Association

North Dakota State Climate Office

North Dakota State Data Center - North Dakota State University

North Dakota State Department of Commerce

North Dakota State University, Department of Agriculture

North Dakota State Water Commission

\section{Ohio}

Ohio Department of Natural Resources 


\section{Oklahoma}

City of Oklahoma City-Water \& Wastewater Utilities

City of Tulsa-Department of Public Works

Grand River Dam Authority

National Weather Service

Oklahoma Agricultural Statistics Service

Oklahoma Climatological Survey

Oklahoma Corporation Commission

Oklahoma Department of Commerce

Oklahoma Department of Environmental Quality

Oklahoma Municipal Power Authority

Oklahoma Water Resources Board

Southwest Power Administration

\section{Oregon}

Oregon Golf Association

Oregon Health Authority Drinking Water Services

Oregon State University Extension Service

Oregon Water Resources Department

\section{Pennsylvania}

Delaware River Basin Commission

Pennsylvania Department of Environmental Protection

\section{Puerto Rico}

Puerto Rico Aqueduct and Sewer Authority

Puerto Rico Department of Agriculture

Puerto Rico Department of Health

Puerto Rico Department of Natural and Environmental Resources

Puerto Rico Electric and Power Authority

Puerto Rico Land Authority

\section{Rhode Island}

Rhode Island Department of Environmental Management

Rhode Island Department of Health

Rhode Island Water Resources Board

\section{South Carolina}

Duke Energy Corporation

Santee Cooper Power

South Carolina Department of Health and Environmental Control

South Carolina Electric and Gas

\section{South Dakota}

South Dakota Department of Agriculture

South Dakota Department of Environment and Natural Resources

Tennessee

Memphis Light, Gas and Water

Tennessee Department of Environment and Conservation, Division of Water Resources

Tennessee Valley Authority

U.S. Army Corps of Engineers

\section{Texas}

Texas Commission on Environmental Quality

Texas Railroad Commission

Texas Water Development Board-Water Use and Projections \& Planning

\section{U.S. Virgin Islands}

U.S. Virgin Islands Water and Power Authority

\section{Utah}

State of Utah Automated Geographic Reference Center

Utah Department of Agriculture

Utah Department of Natural Resources, Division of Oil, Gas, and Mining

Utah Department of Natural Resources, Division of Water Resources

Utah Department of Natural Resources, Division of Water Rights

Utah Department of Natural Resources, Division of Wildlife Resources

\section{Vermont}

Champlain Water District

Vermont Agency of Natural Resources, Department of Environmental Conservation

Vermont Golf Course Superintendents Association

\section{Virginia}

Virginia Department of Environmental Quality, Water Supply Planning Program

Virginia Department of Health, Office of Drinking Water

\section{Washington}

Washington State Department of Ecology

Washington State Department of Health 


\section{West Virginia}

West Virginia Department of Environmental Protection, Division of Water And Waste Management

West Virginia Department of Health and Human Resources, Bureau for Public Health

West Virginia Geological and Economic Survey

\section{Wisconsin}

Public Service Commission of WisconsinWater and Energy Divisions

Wisconsin Department of Natural Resources

\section{Wyoming}

Wyoming Agricultural Statistics Service

Wyoming Department of Employment

Wyoming Oil and Gas Conservation Commission

Wyoming Water Development Commission 
Manuscript approved on September 24, 2014

Prepared by the USGS Science Publishing Network Raleigh Publishing Service Center (PSC)

Edited by Kay P. Naugle

Illustrations and layout by Caryl J. Wipperfurth

Cover by Carol A. Quesenberry, Denver PSC

For more information about this publication contact: U.S. Geological Survey

National Water Use Program

wu-info@usgs.gov

http://water.usgs.gov/watuse/ 


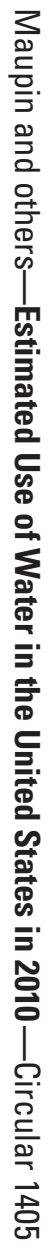

